Florida International University

FIU Digital Commons

6-30-2020

\title{
Structure, Control, and Communication of Collective Animal Behavior in Dynamic Environments
}

Ivan Ignacio Rodriguez-Pinto

Florida International University, irodr171@fiu.edu

Follow this and additional works at: https://digitalcommons.fiu.edu/etd

Part of the Behavior and Ethology Commons, and the Marine Biology Commons

\section{Recommended Citation}

Rodriguez-Pinto, Ivan Ignacio, "Structure, Control, and Communication of Collective Animal Behavior in Dynamic Environments" (2020). FIU Electronic Theses and Dissertations. 4463.

https://digitalcommons.fiu.edu/etd/4463

This work is brought to you for free and open access by the University Graduate School at FIU Digital Commons. It has been accepted for inclusion in FIU Electronic Theses and Dissertations by an authorized administrator of FIU Digital Commons. For more information, please contact dcc@fiu.edu. 


\section{FLORIDA INTERNATIONAL UNIVERSITY}

Miami, Florida

\section{STRUCTURE, CONTROL, AND COMMUNICATION OF COLLECTIVE ANIMAL BEHAVIOR IN DYNAMIC ENVIRONMENTS}

A dissertation submitted in partial fulfillment of

the requirements for the degree of

DOCTOR OF PHILOSOPHY

in

BIOLOGY

by

Ivan Ignacio Rodriguez-Pinto 
To: Dean Michael R. Heithaus

College of Arts, Sciences and Education

This dissertation, written by Ivan Ignacio Rodriguez-Pinto, and entitled Structure, Control, and Communication of Collective Animal Behavior in Dynamic Environments, having been approved in respect to style and intellectual content, is referred to you for judgment.

We have read this dissertation and recommend that it be approved.

Nils Olav Handegard

Yannis Papastamatiou

Jamie C. Theobald

Wei Zeng

Kevin M. Boswell, Major Professor

Date of Defense: June 30, 2020

The dissertation of Ivan Ignacio Rodriguez-Pinto is approved.

Dean Michael R. Heithaus College of Arts, Sciences and Education

Andrés G. Gil

Vice President for Research and Economic Development and Dean of the University Graduate School

Florida International University, 2020 
(C) Copyright 2020 by Ivan Ignacio Rodriguez-Pinto

All rights reserved. 


\section{DEDICATION}

For my parents, who raised me with a strong sense of intellectual curiosity and supported me during each and every step of the process. For my sister, who helped me focus my efforts and encouraged me during the difficult times. For my partner Sonja, my greatest teammate who gave me purpose and kept me going every single day. 


\section{ACKNOWLEDGMENTS}

I would like to thank a multitude of people who supported me on my journey towards completing this dissertation. On the academic side, I would like to thank my major professor, Kevin Boswell, who provided me with exceptional mentorship and training in understanding the biophysical world, as well as an environment to pursue my interests in the quantitative sciences. I would also like to thank my committee: Dr. Handegard, who taught me novel ways of thinking and approaching problems- Dr. Zeng, who supported me via years of training in computational geometry and computer visionDr. Papastamatiou, who provided me excellent insight into predator-prey ecology- and Dr. Theobald, my earliest mentor and best friend who cultivated my love for science and inspired me to become a scientist. On the personal side, I would like to thank Carlos Ruiz, who began this journey with me and helped me weather all of the ups and downs of graduate school, and Krystine Pimentel, Michelle Pierre, and John Gibson, my support system from the $\mathrm{BD} / \mathrm{McKnight}$ program that helped me navigate my transition to FIU and focus my efforts to complete my dissertation. I would also like to thank my colleagues from the Marine Science Program, James Kilfoil and Nan Yao, who accompanied me on this journey and were instrumental to my progress, as well as my research assistants, Savannah Labua, Andrea Rice, and Melissa Johnson for helping me collect and analyze data for this dissertation. I would also like to thank the administrators and supervisors who supported me during my time at FIU: Sonja Montas-Hunter, Shekhar Bhansali, Lidia Kos, as well as John Geiger, Yuying Zhang, Joel Trexler, and Marcy Kravec. Finally, I want to thank Dr. Guillaume Rieucau, who was instrumental in helping me finish these projects; without his guidance on the intricacies of behavioral 
ecology, schooling behavior, and publishing, this dissertation would not have been completed.

I would also like to acknowledge the internal and external funding sources that allowed me to complete this dissertation: The National Science Foundation FGLSAMP@FIU Bridge to the Doctorate Fellowship (HRD \#1301998), FIU Dissertation Year Fellowship, and the Florida Education Fund McKnight Dissertation Fellowship. 


\title{
ABSTRACT OF THE DISSERTATION \\ STRUCTURE, CONTROL, AND COMMUNICATION OF COLLECTIVE ANIMAL BEHAVIOR IN DYNAMIC ENVIRONMENTS
}

\author{
by
}

Ivan Ignacio Rodriguez-Pinto

Florida International University, 2020

Miami, Florida

\section{Professor Kevin M. Boswell, Major Professor}

Collective behavior in animal aggregations is highly complex and spans multiple spatial scales, across a wide range of environmental conditions. In socially active fish, aggregation into schools is a widespread adaptation that confers a variety of safety benefits. The emergent patterns exhibited by collectively behaving fish schools may be influenced by biotic (i.e., predation) or abiotic (i.e., habitat complexity, turbidity) factors in the local environment. Our knowledge of the ways and extent to which environment variability affects schooling behavior at the collective level is currently limited. In my dissertation, I investigated whether environmental factors influenced the collective behavior of fish schools. I focused on three main questions: (1) does habitat context modulate the collective state of fish schools? (2) does the simultaneous presence of habitat structure and high predation risk influence the collective state of fish schools?

(3) do environmental constraints on visual sensory perception affect collective responses to predation? Using advanced field methods to observe schooling behavior in restrictive environmental contexts, question one demonstrated that habitat context, not predation, drives change in the collective state of fish schools, suggesting that the local environment 
plays a large role than predation risk in structuring collective behavior. Using the same field method at an anthropogenic structure containing both habitat complexity and increased predation risk, question two shows that collective behavior is influenced by the coupling of the two conditions, in a different manner than with habitat complexity alone. Finally, using a behavioral arena that creates three dimensional virtual environments, question three showed that mechanosensory information becomes prioritized when visual perception is compromised, and that multiple sensory systems can control schooling behavior in conditions where information about the environment is unreliable. As a collective itself, the results of these questions advance our understanding of the role the environment plays on influencing collective animal behavior, and offers insights into this understanding from both a mechanistic and process based perspective. 


\section{TABLE OF CONTENTS}

CHAPTER

PAGE

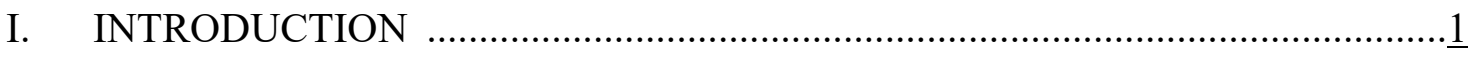

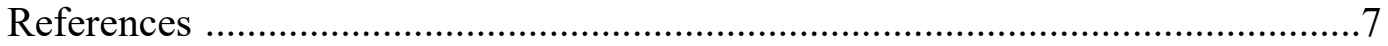

II. ENVIRONMENTAL CONTEXT ELICITS BEHAVIORAL MODIFICATION OF COLLECTIVE STATE IN SCHOOLING FISH ……………....................11

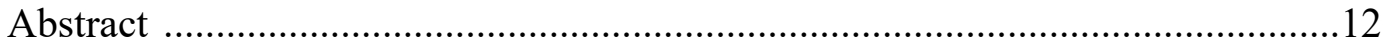

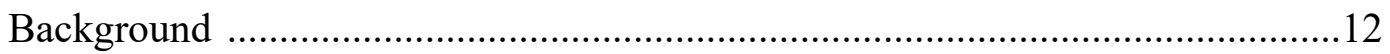

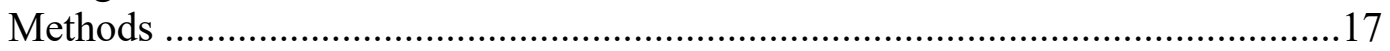

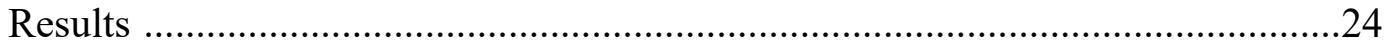

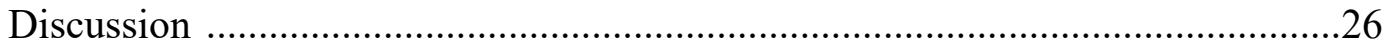

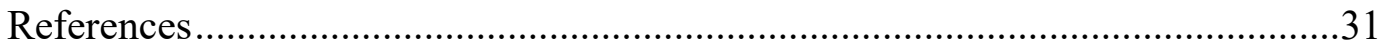

III. PREDATION-HABITAT COMPLEXITY COUPLING ALTERS

COLLECTIVE STATE IN SCHOOLING FISH ………................................47

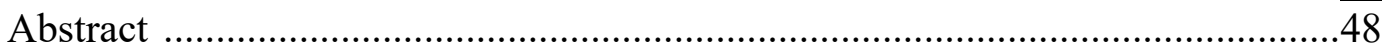

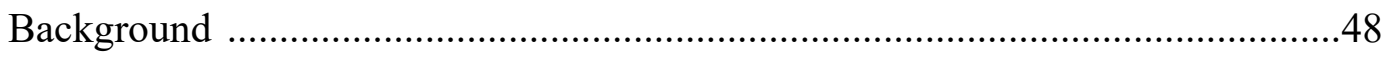

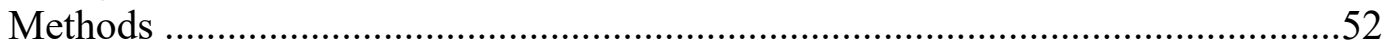

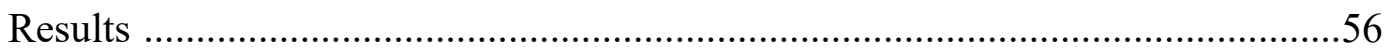

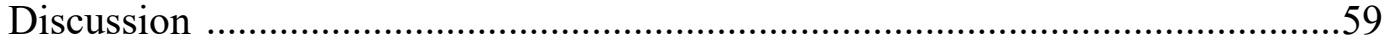

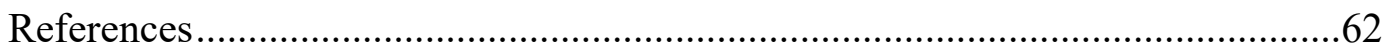

IV. ENVIRONMENTAL IMPACTS ON VISUAL PERCEPTION MODULATES BEHAVIORAL RESPONSES OF SCHOOLING FISH TO LOOMING

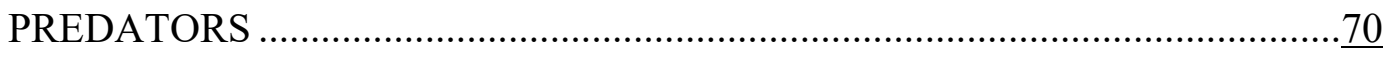

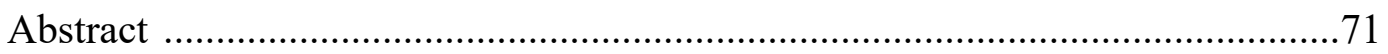

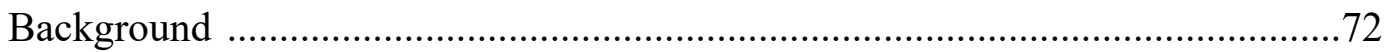

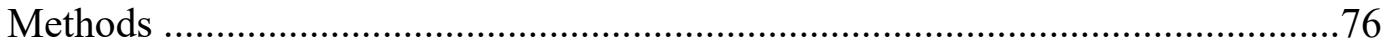

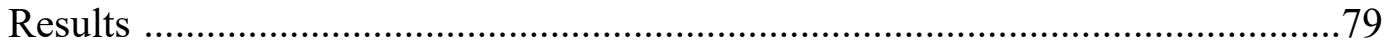

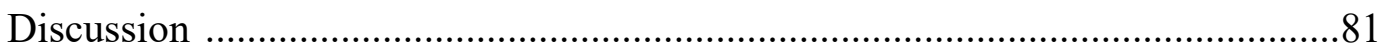

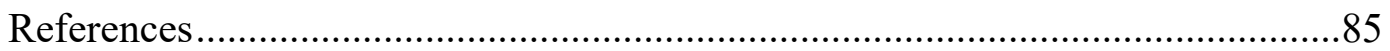

V. CONCLUSIONS \& FUTURE DIRECTIONS …………………........................

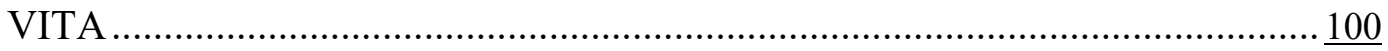




\section{LIST OF TABLES}

CHAPTER

PAGE

\section{CHAPTER II}

Table (1): Two-way MANOVA table comparing the effect of Predation, Habitat, and their interaction among the six schooling state variables

Table (2): Univariate ANOVA tables for each schooling state metric, comparing effects of Predation, Habitat, and interaction.............................................................. 44

Table (3): Tukey post-hoc pairwise comparisons testing between each habitat, separating between pre-and mid-attack

Table (A1): PCA variable loadings for each habitat and attack condition. Schooling state parameter measurements are denoted in the first column, with principal components denoted along the first row. Scree plot analysis show that the first four principal components explain $>95 \%$ of the variability in the data

\section{CHAPTER III}

Table (1): Two-way ANOVA results with subsequent post-hoc pairwise comparisons for each of the six schooling metrics describing schooling tendency. ANOVA tables testing for effect of habitat context, predation, and their interaction are reported above, while Tukey post-hoc pairwise comparisons within habitat context and predation conditions are reported below.

\section{CHAPTER IV}

Table (1): PCA variable loadings for each contrast condition. Schooling state parameter measurements are denoted in the first column, with principal components denoted along the first row. Scree plot analysis show that the first three principal components explain $>95 \%$ of the variability in the data 


\section{LIST OF FIGURES}

\section{CHAPTER II}

Figure (1): Illustration of marsh pond sample area in Louisiana, USA. Three sonar cone positions represent the three habitat conditions surveyed. Each surveyed condition across the pond was at a shallow depth $(<2 \mathrm{~m})$, with no significant tidal currents present. The free-field condition contained a sediment bottom, free of structure and obstacles. The marsh edge condition is equivalent to the free-field condition with the exception of an edge boundary (outlined in white) that impedes movement of swimming organisms. The habitat complexity condition was comprised of a sediment bottom, but also contained rocks, wood pilings, crab traps, and detritus that served as obstacles toward movement (outlined in white). The habitat complexity condition did not contain an edge boundary so each direction of movement was preserved

Figure (M1): Filtered acoustic image with denoising and background subtraction. Denoising was performed using wavelet-based shrinkage methods; background estimates for each behavioral interaction used the $30^{\text {th }}$ percentile for each pixel intensity across all frames. Once each background is estimated, it is subtracted from each denoised frame in the behavioral interaction

Figure (M2): Particle Image Velocimetry (PIV) computed over a 32x32 pixel window to extract velocity estimates for each frame in the behavioral interaction. PIV was computed for the entire image, using changes in image intensity to estimate velocity magnitude and direction. PIV vectors were filtered temporally over five frame intervals, and further filtered spatially using a $3 \times 3$ pixel window median filter

Figure (M3): Image filtering using Otsu's method and pixel area-based morphological filtering of small objects to isolate fish schools in each acoustic image. The processed image retains the main fish schools in the image and is ready for further processing to extract the boundary of the school of interest

Figure (M4): School regions isolated by segmenting each acoustic image using cluster analysis to distinguish fish schools. The processed image was converted to a two dimensional point cloud, and schools were identified using Euclidean distance (L2 norm) clustering at a distance of $0.3 \mathrm{~m}$. Individual schools detected are denoted by color, with red asterisks representing each schools' center of mass 
Figure (M5): Boundary optimization of detected school cluster with associated cost functions for area and perimeter. The detected school of interest was registered using alpha shapes filtering, and cost functions ascribed to area and perimeter were used to derive the optimum value for the alpha radius

Figure (M6): Final detected school boundary with representative PIV velocity estimates used for analysis of schooling state. The optimized alpha shape school boundary was used to filter the PIV velocity field to include vectors located within the school, providing an accurate estimate of individual behavior within the school. Each school was tracked frame by frame via a Kalman filter over the course of the behavioral interaction.

Figure (2): Comparison of behavioral schooling metrics before and during predator attack over an environmental gradient. All conditions pre-, and during predator attack are reported for each habitat context, and are color coded within each habitat type. Results show mean values of each schooling state metric, with data distributions represented underneath. Error bars were determined via the standard error, and significant differences between habitat contexts are denoted by the asterisk. Six schooling state parameters were quantified, prior to, and during predator attack, across three environmental contexts, for a total of 36 unique measurements.

Figure (3): PCA variable loading plots for each habitat context and predation status condition. PCA was scaled to unit variance, with no rotation. The two largest principal components (all captured $>75 \%$ of the variability) were represented on the $\mathrm{x}$ and $\mathrm{y}$ axes. The percent of the variability explained by each principal component is denoted next to the name.

\section{CHAPTER III}

Figure (1): Schematic representation of the study site along with the habitat conditions where behaviors were observed. The two test sites are denoted in the larger scale map by a star, with each habitat illustrated in the adjacent panel. The marsh creek acted as a free-field, lower predation risk control environment. The water control structure acts as an ecological hotspot, with increased predation risk in addition to the habitat structural complexity.

Figure (2): Mean comparisons of six behavioral metrics that describe schooling tendency between habitat context and predator attack. Habitat context comparisons, separated on the bottom axis are separated between pre and mid predator attack (separated by color), presenting comparisons between and within habitat context. Significant differences at $95 \%$ confidence level are denoted with an asterisk. 
Figure (3A, 3B): Generalized Linear Model fits with identity link functions for relevant pairs of schooling metrics. Fit lines and associated correlation coefficients were calculated from the GLM model for each combination of habitat context and predator attack status. Habitat context data are separated by color, and predation status data are denoted by data point shape. Model fits for habitat context are also separated by color, and model fits for predator attack are differentiated by solid and dashed lines. Significant fits and associated correlation values are reported in the upper right-hand corner of each plot.

\section{CHAPTER IV}

Figure (1): Experimental apparatus visualizing fish school in three dimensional virtual arena. Each school was subjected to four consecutive visual looming stimuli, with randomized direction, at a given contrast level. Once schools completed the experiment, they were returned to the tank. Subsequent trial sample randomly to ensure no two schools are exactly the same.

Figure (2): Mean values of five behavioral parameters in schooling minnow across four varying contrast levels, denoted by increasingly lighter color. Error bars represent standard error, and jitter points represent the raw values. Nearest neighbor distance, nearest neighbor alignment angle, and swimming speed were calculated from each fish in the school across the entire behavioral response time and averaged to provide one measurement per school tested. Polarization and Correlation strength were calculated per school, averaging across the entire time span of each behavioral response, providing one measurement per school tested.

Figure (3): Evolution of five behavioral parameters in schooling minnow (species) along the time span of response to a looming stimulus across four contrast levels. The time span of the behavioral response varied per trial and were standardized by partitioning each behavioral response time span into 10 equidistant time intervals. Solid lines represent mean values of parameters, with contrast denoted by increasingly lighter color; standard error is represented by a similarly hued region bounding the mean value line.

Figure (4): Principal Components Analysis of the five behavioral parameters of schooling minnow for each contrast level. Variable loadings on the first two principal components are shown for each contrast level tested. Each PCA was scaled to unit variance. 


\section{PREFACE}

The following chapter has been accepted for publication and has been formatted for that volume.

\section{CHAPTER II}

Rodriguez-Pinto, I., Rieucau, G., Handegard, NO., and Boswell, KM., "Environmental Context Elicits Behavioural Modification of Collective State in Schooling Fish" (2020) Animal Behaviour 


\section{CHAPTER I}

INTRODUCTION 
Organismal aggregation and collective behavior are unique pattern-forming phenomena that exist in the natural world on a multitude of spatiotemporal scales, from short lived swarms of locusts and cicadas, to flocks of starling dancing in the sky while the sun sets over the horizon. Collective behavior in living organisms is largely a result of social interactions among conspecific animals, in which an aggregation response functions as an adaptation for improved foraging success, mobility, or avoiding predation (Krause and Ruxton, 2002). Collective behavior arises in species that vastly differ in their evolutionary complexity (i.e, insects, fish), as well as in widely different physical conditions (i.e, water, air), demonstrating that group living and collective motion confer strong evolutionary benefits (DeLellis et al., 2015). In addition, the behavioral patterns and underlying mechanisms that govern these patterns then create an optimal strategy to maximize fitness in these different species; understanding the nature of these mechanisms can help us decipher how these patterns appear in across such a large spatiotemporal gradient. The underlying drivers of collective motion result in a unique paradigm; the collective exists without a leader, a geometrical constraint, or an external field that guides group formation (Deutsch et al., 2012). A major question in this field is then: How are these patterns formed, what ecological purpose do they serve, and how are they influenced by external factors? An attempt to answer these questions then rely on conceptualizing living groups as self-organized systems; investigation of internal state, input/output sensory components that drive state trajectories, and responses to perturbations of self-organized systems (Wiener, 2019) can inform how these animal groups behave collectively. 
Socially active fishes, in particular, utilize aggregation to form schools that primarily provide security advantages. These security advantages come in the form of increased predator detection (Lima, 1998), predator confusion (Ioannou and Krause, 2008), dilution of predation risk because of large numbers, and rapid coordinated evasive motion (Parrish et al., 2002; Partridge et al., 1980). In a fish school, each individual responds to its local environment as well as the behavior of its neighbors, a process able to elicit emergent collective responses (Radakov, 1973; Seghers, 1974; Viscido et al., 2004). The coordinated maneuvers of the collective response allow for a multitude of rapid evasive reactions to a direct predator attack which range from flash expansion to the fountain effect (Parrish, 1993; Pitcher and Parrish, 1993). The underlying interaction mechanisms that control the behavioral "state" of a freely-behaving fish school are very complex have been studied in both simulation and laboratory settings; simplified interaction models have been proposed to simulate how an individual in the aggregation behaviorally responds to its immediate neighbors (Couzin, 2009; Couzin et al., 2002; Couzin et al., 2006; Katz et al., 2011), which include positioning/speed within the school, as well as alignment between individuals. Mechanosensory (via the lateral line), visual (via eyes), and chemical cues (via olfactory receptors) are used to drive the collective behavioral response, which depends on distributed sensing at the individual level (Partridge and Pitcher, 1980). For example, a predatory shark attacking a school is perceived by an individual fish at the edge of the school, which modulates that individual fish's behavior. The individual motion made in response to perceiving the predator creates a perturbation of the local environment, thus a gradient of sensory stimuli forms in the water immediately surrounding the individual. The neighboring fish perceive the 
changing sensory gradient and a collective antipredator response occurs (Herbert-Read et al., 2015; Rieucau et al., 2016; Ward et al., 2008; Ward et al., 2011). During the schooling response, fish receive the maximum safety benefit conferred by schooling when the information about the predator reaches all of the individuals before the predator reaches the school (Magurran and Higham, 1988). Quantification and understanding of the internal behavioral state of fish schools are a necessary step to a thorough understanding of collective behavior.

In fish, multiple sensory modalities exist that determine their behavior. From a self-organized system perspective, these sensory systems represent the input/output components that modulate the internal state of the fish school. The visual and lateral line systems are the primary means by which individuals mediate distance and orientation to neighbors (Faucher et al., 2010). The visual systems of fish are comparable to those of mammals, and can perceive colors as well as UV light (Pita et al., 2016). The cameratype eyes allow for a high spatiotemporal resolution and sensitivity, up to a certain point bounded by photoreceptor size and density, which determines the diffraction limit (Land, 2005). The lateral line system consists of ciliated cells known as neuromasts; within each neuromast are bundles of hair cells, which transduce mechanical energy such as water flow or local acceleration to nerve signals (Coombs and Montgomery, 1999; Goulet et al., 2012). Within a school, the visual system mediates attraction to a neighbor, as well as position and orientation; the lateral line system mediates repulsion from neighbors, and regulates velocity, direction of travel, and rheotaxis (Paciorek and McRobert, 2013). These sensory systems as well as the internal state of a freely-behaving fish school have been shown to be influenced by variation in their local environment (Berdahl et al., 2013; 
Brown et al., 2006; Crane et al., 2019; Domenici et al., 2002), so an understanding of whether (and how) environmental conditions perturb the collective state of a fish school can extend our understanding of the collective behavior phenomenon.

Physically dominated (i.e., tidally driven) coastal ecosystems display considerable variation in hydrodynamic properties that can span large spatial (m-km) and temporal scales (minutes-years). Of particular interest in coastal ecosystems is the impact of predictable and fundamental regime shifts in important abiotic factors from tidal forcing (e.g., turbidity, tidal mixing, water quality, and velocity) and anthropogenic (floodwater management, pier/bridge/dam construction) activities in structuring these processes in coastal ecosystems. Specifically, water turbidity and habitat characteristics can reduce prey's ability to perceive the presence of a predator (Abrahams and Kattenfeld, 1997; Ferrari et al., 2010), structural complexity can restrict potential directions of escape (Kimball et al., 2015), and fluctuations in hydrodynamic conditions in tidally-driven estuarine ecosystems can contribute to weakened perception of predation cues and the overall risk of predation (Lunt and Smee, 2014; Smee et al., 2008). Predators can impact the distribution and behavior of prey organisms through direct consumption, as well as indirect processes termed non-consumptive effects (Menge, 1995; Peckarsky et al., 2008; Preisser et al., 2005). It is becoming increasingly clear that the role of non-consumptive effects is equally important in structuring ecological processes, and the role of predators acting simply as 'agents of fear' can have cascading consequences throughout the ecosystem (Preisser et al., 2005; Preisser et al., 2008). In marine ecosystems, it is likely that dominant schooling fish species must adopt strategies to balance the ever-present and variable risk effects, 'Landscape of Fear' (Brown et al., 1999), with foraging and energetic 
demands. As a group, schooling fish species must then be able to preserve their ability to communicate at the group level to provide and perceive information on risk effects in the face of environmental variability. Seascape variation can dramatically affect how efficiently and accurately aquatic organisms evaluate risk effects or identify threats (Chivers et al. 2001; Brown et al. 2006) and, from a collective perspective, may affect the ability for information about a threat to propagate to other fish in the school. Therefore, collective behavior of schooling fish may be susceptible to temporally variable environmental conditions where signals from both visual and mechanosensory modalities at the individual level may be confounded.

The goal of my dissertation is to investigate the impact of local environmental conditions on the collective behavior of fish schools. Evaluating and quantifying the role of environmental changes on schooling behavior has in practice been difficult largely as a consequence of the logistical challenges of working in dynamic environments and the capacity of traditional methods to capture these processes at relevant temporal and spatial scales. I used a combination of advanced field and laboratory methods to observe schooling behavior in various environmental contexts and assessed their impact from both a group and individual level perspective. In chapter II, I address the question of whether habitat context modulates collective behavior in schooling fish. I quantify the behavioral state of fish schools prior to, and during, predator attack across three different habitat contexts that varied in complexity in an estuarine system. In chapter III, I build upon the results of chapter II and address the question of whether the combination of high predation risk and habitat complexity influence the collective behavior of fish schools. To answer whether the coupling of increased predation risk and habitat complexity affect 
collective behavior, I quantify the behavioral state of fish schools prior to, and during, predator attack in an open marsh canal and at a water control structure, an area that acts as an aggregative hotspot, exhibiting higher predation risk in addition to structural complexity. Finally, in chapter IV, I return to the laboratory for a finer-scale investigation of whether environmental influences on visual perception affect schooling escape responses to looming predators. To answer whether contrast decreases affect collective responses to predators, I used a three-dimensional virtual environment to observe schooling responses to looming optical stimuli under four differing contrast levels. Overall, this work advances the field by further expanding our knowledge of the drivers, mechanisms, and ecology governing collective animal behavior.

\section{References}

Abrahams, M. V. and Kattenfeld, M. G. (1997). The role of turbidity as a constraint on predator-prey interactions in aquatic environments. Behavioral Ecology and Sociobiology 40, 169-174.

Berdahl, A., Torney, C. J., Ioannou, C. C., Faria, J. J. and Couzin, I. D. (2013). Emergent sensing of complex environments by mobile animal groups. Science 339, 574576.

Brown, J. S., Laundré, J. W. and Gurung, M. (1999). The ecology of fear: optimal foraging, game theory, and trophic interactions. Journal of Mammalogy 80, 385 399.

Brown, G. E., Rive, A. C., Ferrari, M. C. O. and Chivers, D. P. (2006). The dynamic nature of antipredator behavior: prey fish integrate threat-sensitive antipredator responses within background levels of predation risk. Behavioral Ecology and Sociobiology 61, 9-16.

Coombs, S. and Montgomery, J. C. (1999). The enigmatic lateral line system. In Comparative hearing: Fish and amphibians, pp. 319-362. Springer. 
Couzin, I. D. (2009). Collective cognition in animal groups. Trends in Cognitive Sciences $13,36-43$.

Couzin, I. D., Krause, J., James, R., Ruxton, G. D. and Franks, N. R. (2002). Collective memory and spatial sorting in animal groups. Journal of theoretical biology 218, $1-11$.

Couzin, I. D., James, R., Mawdsley, D., Croft, D. P. and Krause, J. (2006). Social organization and information transfer in schooling fishes. Fish cognition and behavior 166-185.

Crane, A. L., Ferrari, M. C. O., Rivera-Hernández, I. A. E. and Brown, G. E. (2019). Microhabitat complexity influences fear acquisition in fathead minnows. Behavioral Ecology.

DeLellis, P., Polverino, G., Ustuner, G., Abaid, N., Macrì, S., Bollt, E. M. and Porfiri, M. (2015). Collective behaviour across animal species. Scientific Reports 4,.

Deutsch, A., Theraulaz, G. and Vicsek, T. (2012). Collective motion in biological systems. Interface Focus 2, 689-692.

Domenici, P., Silvana Ferrari, R., Steffensen, J. F. and Batty, R. S. (2002). The effect of progressive hypoxia on school structure and dynamics in Atlantic herring Clupea harengus. Proceedings of the Royal Society B: Biological Sciences 269, 21032111.

Faucher, K., Parmentier, E., Becco, C., Vandewalle, N. and Vandewalle, P. (2010). Fish lateral system is required for accurate control of shoaling behaviour. Animal Behaviour 79, 679-687.

Ferrari, M. C. O., Lysak, K. R. and Chivers, D. P. (2010). Turbidity as an ecological constraint on learned predator recognition and generalization in a prey fish. Animal Behaviour 79, 515-519.

Goulet, J., van Hemmen, J. L., Jung, S. N., Chagnaud, B. P., Scholze, B. and Engelmann, J. (2012). Temporal precision and reliability in the velocity regime of a hair-cell sensory system: the mechanosensory lateral line of goldfish, Carassius auratus. Journal of Neurophysiology 107, 2581-2593.

Herbert-Read, J. E., Buhl, J., Hu, F., Ward, A. J. and Sumpter, D. J. (2015). Initiation and spread of escape waves within animal groups. Royal Society open science 2, 140355.

Ioannou, C. C. and Krause, J. (2008). Searching for prey: the effects of group size and number. Animal Behaviour 75, 1383-1388. 
Katz, Y., Tunstrøm, K., Ioannou, C. C., Huepe, C. and Couzin, I. D. (2011). Inferring the structure and dynamics of interactions in schooling fish. Proceedings of the National Academy of Sciences 108, 18720-18725.

Kimball, M. E., Rozas, L. P., Boswell, K. M. and Cowan Jr, J. H. (2015). Effects of slotted water control structures on nekton movement within salt marshes. Marine and Coastal Fisheries 7, 177-189.

Krause, J. and Ruxton, G. D. (2002). Living in groups. Oxford University Press.

Land, M. F. (2005). The optical structures of animal eyes. Current Biology 15, R319R323.

Lima, S. L. (1998). Nonlethal effects in the ecology of predator-prey interactions. Bioscience 48, 25-34.

Lunt, J. and Smee, D. L. (2014). Turbidity influences trophic interactions in estuaries. Limnology and Oceanography 59, 2002-2012.

Magurran, A. E. and Higham, A. (1988). Information transfer across fish shoals under predator threat. Ethology 78, 153-158.

Menge, B. A. (1995). Indirect Effects in Marine Rocky Intertidal Interaction Webs: Patterns and Importance. Ecological Monographs 65, 21.

Paciorek, T. and McRobert, S. (2013). Daily shoaling patterns in the zebrafish Danio rerio. Current Zoology 59, 754-760.

Parrish, J. K. (1993). Comparison of the Hunting Behavior of Four Piscine Predators Attacking Schooling Prey. Ethology 95, 233-246.

Parrish, J. K., Viscido, S. V. and Grünbaum, D. (2002). Self-organized fish schools: an examination of emergent properties. The biological bulletin 202, 296-305.

Partridge, B. L. and Pitcher, T. J. (1980). The sensory basis of fish schools: relative roles of lateral line and vision. Journal of Comparative Physiology 135, 315-325.

Partridge, B. L., Pitcher, T., Cullen, J. M. and Wilson, J. (1980). The three-dimensional structure of fish schools. Behavioral Ecology and Sociobiology 6, 277-288.

Peckarsky, B. L., Abrams, P. A., Bolnick, D. I., Dill, L. M., Grabowski, J. H., Luttbeg, B., Orrock, J. L., Peacor, S. D., Preisser, E. L., Schmitz, O. J., et al. (2008). Revisiting the classics: considering nonconsumptive effects in textbook examples of predator-prey interactions. Ecology 89, 2416-2425.

Pita, D., Collignon, B., Halloy, J. and Fernández-Juricic, E. (2016). Collective behaviour in vertebrates: a sensory perspective. R. Soc. open sci. 3, 160377. 
Pitcher, T. J. and Parrish, J. K. (1993). Functions of schooling behavior in fishes. The behavior of teleost fishes. Chapman and Hall, New York 363-440.

Preisser, E. L., Bolnick, D. I. and Benard, M. F. (2005). Scared to death? The effects of intimidation and consumption in predator-prey interactions. Ecology 86, 501-509.

Preisser, E. L., Orrock, J. L. and Schmitz, O. J. (2008). Predator hunting mode and habitat domain alter nonconsumptive effects in predator-prey interactions.

Radakov, D. V. (1973). Schooling in the ecology of fish.

Rieucau, G., Holmin, A. J., Castillo, J. C., Couzin, I. D. and Handegard, N. O. (2016). School level structural and dynamic adjustments to risk promote information transfer and collective evasion in herring. Animal Behaviour 117, 69-78.

Seghers, B. H. (1974). Schooling Behavior in the Guppy (Poecilia reticulata): An Evolutionary Response to Predation. Evolution 28,.

Smee, D. L., Ferner, M. C. and Weissburg, M. J. (2008). Alteration of sensory abilities regulates the spatial scale of nonlethal predator effects. Oecologia 156, 399-409.

Viscido, S. V., Parrish, J. K. and Grünbaum, D. (2004). Individual behavior and emergent properties of fish schools: a comparison of observation and theory: Emergent properties of complex marine systems: a macroecological perspective. Marine ecology. Progress series 273, 239-249.

Ward, A. J., Sumpter, D. J., Couzin, I. D., Hart, P. J. and Krause, J. (2008). Quorum decision-making facilitates information transfer in fish shoals. Proceedings of the National Academy of Sciences 105, 6948-6953.

Ward, A. J. W., Herbert-Read, J. E., Sumpter, D. J. T. and Krause, J. (2011). Fast and accurate decisions through collective vigilance in fish shoals. Proceedings of the National Academy of Sciences 108, E27-E27.

Wiener, N. (2019). Cybernetics or Control and Communication in the Animal and the Machine. MIT press. 
CHAPTER II

ENVIRONMENTAL CONTEXT ELICITS BEHAVIORAL MODIFICATION OF COLLECTIVE STATE IN SCHOOLING FISH 


\begin{abstract}
Pattern formation and collective behavior in animal aggregations is highly complex and occurs across many scales, over a wide range of environmental conditions. The patterns found in collective behavior may be modulated by the environmental habitat the group is located in. Here, we consider whether habitat context influences the collective behavior of fish schools under threat of predation in a dynamic salt marsh system. By comparing collective responses of wild forage fish prior to, and during predator attack across three environmental contexts, we examine whether schooling state is influenced by the habitat fish schools reside in. Our results indicate that habitat context had a much stronger effect on collective state relative to predation. The habitats studied (both a marsh edge habitat and a higher complexity habitat) induce changes in the behavioral state of fish schools compared to a free-field context, which demonstrates an alteration of the collective behaviors performed by the school. This suggests that other ecological factors, such as the local environment, plays a larger role than predation risk in structuring the spatial and temporal group-level patterns found in collective behavior.
\end{abstract}

\title{
Background
}

Organismal aggregations are found around the world at multiple spatial scales, and are as diverse as swarms of locusts, flocks of birds, and schools of fish. Group-living individuals can receive a variety of benefits, such as improved safety, increased foraging, and reproductive success (Ioannou, 2017; Krause and Ruxton, 2002). In an aggregation, such as a fish school, each individual responds to its local environment as well as the behavior of its neighbors, a process which can elicit emergent collective responses 
(Couzin et al., 2006; Herbert-Read et al., 2015), particularly when under threat of predation (Pitcher, 1983; Parrish, 1989; Parrish et al., 2002; Ioannou et al., 2012; Rieucau et al., 2015). Collective responses of schooling fish to predation pressure has been well studied, from the aggregative tendency in the presence of predator cues (Hoare et al., 2004), to the dilution of predation risk (Turner and Pitcher, 1986) and decreasing encounter rate (Ioannou et al., 2011) via grouping. In addition, behavioral patterns in the anti-predator response have also been examined, from the selfish herd mechanism that reduces risk via minimizing distance between conspecifics (Parrish, 1989) and the confusion effect (confusion of predator choice of prey due to prey density) (Jeschke and Tollrian, 2007; Ruxton et al., 2007), to the mechanisms of collective vigilance (HerbertRead et al., 2015; Herbert-Read et al., 2017a; Ward et al., 2011) and threat sensitive responses (Brown et al., 2006; Rieucau et al., 2014; Rieucau et al., 2016a) that utilize the transferring of social information about the predator cue throughout the group (Magurran and Higham, 1988). However, it has been shown that environmental effects play a role in structuring predator-prey interactions (Abrahams and Kattenfeld, 1997; Cook and Streams, 1984; Crowder and Cooper, 1982; Higham et al., 2015), therefore these types of collective anti-predator responses may be influenced by local environmental biotic and abiotic factors.

In dynamic marine environments with varying physical and hydrological conditions, including regions of habitat complexity, environmental shifts occur at multiple spatial and temporal scales. The wide array of environmental conditions modulate predator-prey interactions in fish via changes in a number of abiotic factors, including turbidity (Ajemian et al., 2015; De Robertis et al., 2003), temperature (Weetman et al., 
1998), and oxygen levels (Domenici et al., 2007). Similarly, schooling behavior (particularly in juveniles and herbivorous species) is ubiquitous across this entire spectrum of environmental conditions, and the social dynamics have been shown to be affected by similar environmental factors, including turbidity (Borner et al., 2015; Kimbell and Morrell, 2015), hypoxia (Domenici et al., 2017), and light level (Ryer and Olla, 1998). The occurrence of schooling across a gradient of environmental conditions suggests robustness in the behavioral schooling state to environmental changes, but may still be altered when coupled with threat of predation. Previous studies have shown how various environmental factors, including turbidity, predation pressure, and noise, affect anti-predator schooling behavior. Turbid water promotes risk-averse behavior and decreases aggregative tendency in favor of individualistic decisions (Chamberlain and Ioannou, 2019), as well as eliciting weaker anti-predator responses and higher dispersion within the group (Kimbell and Morrell, 2015), high predation habitats modulate group decision making (Ioannou et al., 2017) as well as social interactions (Herbert-Read et al., 2017a), and the presence of anthropogenic noise destabilizes school structure (Herbert-Read et al., 2017b). Habitat complexity has also been shown to influence anti-predator behavior and survival (Crowder and Cooper, 1982; Figueiredo et al., 2015; Lichtenstein et al., 2019), and given the variety of influences environmental factors act on schooling behavior, it is still unclear whether habitat context modulates the collective anti-predator response. An understanding of how different environments affect the collective sensing and decision making of a fish school can thus provide insight on the plasticity and robustness of group behavior and contribute valuable information to ecosystem management and conservation efforts. 
In observing freely behaving groups of fish, a variety of motion and interactionbased metrics have been used to quantify the behavioral state of a school (Delcourt and Poncin, 2012). Schooling state metrics that can be derived from behavioral observations include, e.g., the cross-sectional area of the school (Partridge et al., 1980), the average swimming speed (Berdahl et al., 2013; Kent et al., 2019; Zienkiewicz et al., 2018), fish directional polarization (Cavagna et al., 2008; Viscido et al., 2004), angular velocity (Tunstrøm et al., 2013), rotational order (Attanasi et al., 2014a), and correlation strength as a measure of information transfer rate (Attanasi et al., 2014c; Cavagna et al., 2008; Cavagna et al., 2010; Handegard et al., 2012). These metrics have been used to characterize internal schooling states (Tunstrøm et al., 2013), determine the behavioral responses to predator threat and evasion (Rieucau et al., 2016b), analyze the ability to propagate information to conspecifics (Rosenthal et al., 2015; Strandburg-Peshkin et al., 2013), and propose novel models of collective behavior that accurately replicate the emergent patterns found in the collective response (Cavagna et al., 2015; Couzin et al., 2002). The collective response of aggregated animals have been well studied in both simulation and laboratory settings, at both the individual and group-level (Gautrais et al., 2012; Lopez et al., 2012; Radakov, 1973; Rieucau et al., 2016b). However, in situ, remotely observed field level studies, although more difficult to conduct, provide a suitable representation of how the collective behavior occurs in the natural world (Handegard et al., 2012; King et al., 2018; Rieucau et al., 2016b), and may show the effect of external influences unable to be replicated in the laboratory. In this study, we simultaneously compare the anti-predator response to real predators, directly in their natural environments containing habitat contexts that are common to the ecosystem. 
Here, our aim is to identify whether habitat context, prior to and in response to predation, affects the behavioral state of schooling juvenile gulf menhaden (Brevoortia patronus). To achieve this objective, we investigated whether schooling state, quantified via six physical metrics, prior to and in response to predator attack is mediated by three different local habitat contexts within a salt-marsh pond. The three habitat contexts we compared were 1) a free-field, lacking any habitat complexity, 2) a marsh edge habitat, where the topographic profile may restrict potential directions of motion, and 3) a habitat of higher complexity, which contained a static physical structure that encompassed the entire water column. We hypothesize that sensory information derived from the habitat context shapes a fish schools' behavioral state, which elicits changes beyond the inherent range of behavioral plasticity that compensates for predator attack. Specifically, we predict that fish schools located in the marsh edge and complex habitat will exhibit differences in schooling state metrics, particularly area, speed, polarization, and correlation strength, between themselves and relative to the free-field habitat context. The restriction in escape directions, as well as physical obstacles in the environment will cause increased school fragmentation (resulting in smaller school sizes and decreased correlation strength) and increased motion coordination (resulting in higher polarization and group speed) relative to schools in the free-field. In addition, we predict that the sensory information difference in heterogeneous habitats compared to the free-field will affect the correlative relationships between the six metrics representing the schooling state, causing a decrease in correlation between school area, correlation strength, and polarization. 


\section{Methods}

\section{(a) Data Collection:}

We deployed a high-resolution imaging sonar, DIDSON (Sound Metrics, Inc.), attached to a tripod approximately $0.4 \mathrm{~m}$ above the seafloor. In this deployment, the major axis of the beam was oriented at a tilt angle $-0.3^{\circ}$ from parallel to the surface, generating a stationary image of the substrate and areal profile of fish schools across each habitat (Figure 1) (Boswell et al., 2007; Boswell et al., 2019). The DIDSON was operated at a frequency of $1.8 \mathrm{MHz}$, where a 96-beam transducer array created a $28^{\circ}$ by $14^{\circ}$ field of view. There are 512 samples along the acoustic axis, with a resolution of $\sim 2 \mathrm{~cm}$, which generate a 512x96 sample space. Recordings were collected at a frame rate of 8 frames per second, and each frame maps to an image with a range of $10 \mathrm{~m}$.

\section{(b) Sampling:}

Similar to prior studies that focused on observing schooling behavior in situ (Handegard et al., 2012; Rieucau et al., 2016b), behavioral observations were extracted directly from underwater recordings of shallow estuarine salt-marsh ponds (Figure 1) near Empire, Louisiana (29.4095N, 89.6300W), USA, between April 2009 and July 2010. The marsh ponds cover $\sim 40,000 \mathrm{~m}^{2}$ in total area, are intertidal, oligohaline (5-25 ppt), and connect to each other via tidal channels which funnel into a larger water-body (Adam's Bay, LA). Recordings were collected from a total of four ponds, some of which contained both soft substrate and areas of habitat complexity (rocks, logs), creating an adequate experimental setting to compare the influence of environmental context on schooling behavior of estuarine fishes. Five hour recordings were collected from an individual pond 
every two months, over intervals of four consecutive days, at the same point in the monthly and daily tidal cycle. Direct sampling of the region (Garner, 2012; Handegard et al., 2012; Klotzbach, 2013) conducted concurrently with the collection of these data shows that the schools observed (due to size) were juvenile gulf menhaden (Brevoortia patronus), and the predators attacking them were typically (due to size and abundance in area) Atlantic croaker (Micropogonias undulatus), or spotted sea trout (Cynoscion nebulosus), ensuring species parity across all schooling behavioral observations.

All recordings were analyzed, and behavioral interactions between schooling fish and predators were extracted across three habitat conditions present amongst the four ponds. The three regions consisted of an open free-field, a marsh edge, and a complex habitat context. During analysis of pond recordings collected in the marsh edge and complex habitat, behavioral interactions qualified for inclusion only if they occurred within $0.3 \mathrm{~m}$ of the marsh boundary or habitat structure; the threshold distance chosen to ensure that responses were influenced by the presence of the objects. For each interaction identified, the free-swimming behavior of each school prior to attack was included in the recording. Over a span of 35 hours of recording, partitioned in equal time intervals for each habitat type, 56 interactions between schools and predators were identified and extracted in the free-field habitat, 25 in the marsh edge habitat, and 17 in the complex habitat for a total of 98 individual recordings.

Each observed recording of a behavioral interaction was partitioned into pre-and post-predator attack components. During analysis of the sonar video, the image frame that coincides with the predator attacking the school was marked as the partitioning threshold. The partitioning criteria was met when one of two conditions occurred: 1) the 
predator strike directly penetrated the school boundary (identified by the formation of a furrow on the school boundary around the predator), inducing a fast-start escape response from the school, or 2) the school elicited an escape response along the same trajectory as the predator (denoted by movement of the school boundary in the predators' direction of attack), when the predator strike breached the reaction distance of the school (Domenici and Hale, 2019). The attack duration was defined as the time interval from attack to when prey ceased responding to the predator (Pitcher, 1983).

For each observed predator attack, the interval of time corresponding to the attack duration was extracted before and after the partitioning threshold. This ensured that all the behavioral phases were split into equal time intervals $(\sim 3-6 \mathrm{~s}$ before/after $)$ around the partitioning threshold, the first interval representing the pre-predator behavior, and the second representing the mid-attack response. After partitioning, a total of 196 behavioral observations (98 pre-attack, 98 mid-attack) were analyzed across the entire sampling period.

The free-field habitat is characterized by a soft sediment bottom, with a depth range of $0.5-1 \mathrm{~m}$ (Figure 1). The edge habitat is characterized as the region where a field habitat abruptly transitions to a physical barrier (e.g., marsh surface), generally with a shallower depth range of $0.1-0.5 \mathrm{~m}$. The edge is composed of solid soil, and acts as a boundary that spans the school (Figure 1). The complex habitat is a free field habitat, without a boundary, but with either a large rock, or wooden log that encompasses the majority of the water column (Figure 1). 


\section{(c) Data Processing:}

Each DIDSON frame represents a 512 x 96 sample space. The raw samples were wavelet denoised (MATLAB R2014b, Image Processing Toolbox) to remove high frequency artifacts, and converted to distance-based grayscale images via a mapping that accounts for radial beam spread (Figure 1). Image backgrounds were estimated for each behavioral interaction using the $30^{\text {th }}$ percentile for each pixel intensity across all frames in the interaction, and were subsequently subtracted from each image (Figure M1). Particle Image Velocimetry (PIV) calculated over a 32 x 32 pixel window was used to extract velocity estimates for the school (Figure M2), to estimate the swimming speed and correlation strength (Fleet and Weiss, 2006). In addition to the PIV calculations, school boundary detection was done to extract the position of the school, the school area, and isolate the velocity vectors that contribute to the behavior of the school. The PIV velocity vectors were additionally filtered temporally over 5 frames, as well as spatially via a 3 pixel window median filter. The school boundary was obtained via initial thresholding using Otsu's method and pixel area-based filtering (MATLAB Image Processing Toolbox) of small objects in the image to extract a two-dimensional point cloud that represented the school shape (Otsu, 1979) (Figure M3). The point cloud was then segmented and labeled based on cluster analysis with a Euclidean norm distance parameter of $0.3 \mathrm{~m}$ (Figure M4). The school cluster was then registered via an alpha shape filter, which contained an adaptive alpha value based on cost functions attributed to the area and perimeter of the boundary (Figure M5). The detected school boundary was tracked frame by frame via a Kalman filter, and was then approximated as a polygon to constrain the PIV velocity vectors to those pertaining to the fish school (Figure M6). 
We compared observations from the three different habitats, before and during predator attack, to test whether the schooling state is modulated by the environmental structural gradient. The tests are as follows: 1) whether habitat and predator attack affect schooling state in terms of (a) school area occupied, (b) group speed, (c) angular velocity, (d) polarization, (e) rotational order, and (f) correlation strength; 2) effect of habitat context on the relationship between metrics (a-f) that describe the school state.

The school area was calculated based on the school boundary detection algorithm and is a local 2D projection approximation of the total area occupied by the school. In our analysis, we assume constant density of schools, so changes in area represent space utilized as opposed to school size. The group speed was derived by center-of-mass measurements of the school and its change in position with respect to time. Angular velocity was computed from the curl of the PIV vectors, as follows:

$$
\omega=\frac{1}{2}(\nabla \times v),
$$

where $v$ denotes the velocity vectors located within the detected school. Polarization and rotational order were calculated from the formulas provided in (Attanasi et al., 2014b). In this case, the polarization is the Euclidean norm of the sum of the velocity vectors normalized by magnitude (to isolate direction) divided by the total number of vectors, as shown below: 


$$
\Phi=\frac{1}{N}\left|\sum_{i=1}^{N} \frac{\overrightarrow{v_{l}}}{v}\right|
$$

The rotational order, computed as follows:

$$
R=\frac{1}{(M N-p)}\left|\sum_{m, n} \frac{x(m, n) \times v(m, n)}{|x(m, n) \times v(m, n)|} \cdot e_{z}\right|
$$

measures the coherence of rotation in the school, quantified by summing the rotational components (projection of each rotational vector onto the axis orthogonal to the sonar plane, $e_{z}$, relative to the center of the school) divided by its magnitude, and normalized by the number of vectors (fish) within the school. Integrated correlation strength (the degree to which one fish's behavior influences its neighbors) was calculated in the same manner as (Rieucau et al., 2016b), and formulated below:

$$
c_{k i}=\int_{d=0}^{4 m} c(d) \mathrm{d} d
$$

where $c(d)$ was the correlation strength for a given frame $i$ that measured how the behavioral change of an individual affects their neighbors as a function of distance. The correlation strength for distances greater than $4 \mathrm{~m}$ were nearly zero, so it was used as the upper bound of the integration. The correlation strength for each frame was calculated 
from the spatial autocorrelation of the velocity fluctuations of each school, and mapped to metric distance in the sonar image (Handegard et al., 2012; Rieucau et al., 2016b).

\section{(d) Statistical Analysis:}

In the analysis of the behavioral interactions, each individual school is treated as an independent sample, and residual analysis (via QQ-plot) maintained the assumption of normality. To determine whether predation, habitat, or their interaction explained the variance in the data, we conducted a two-way MANOVA on group speed, school area occupied, angular velocity, polarization, rotational order, and correlation strength. We subsequently conducted univariate ANOVA's to test within each schooling metric. Tukey post-hoc pairwise comparisons were conducted to determine significance (at $95 \%$ significance level) of the mean schooling metrics within predation, habitat, and interaction factors, corrected (Bonferroni) for multiple comparisons. In addition, Principal Component Analysis (PCA), scaled to unit variance, was done to examine the correlation between the schooling metrics before and during predator attack, for each habitat context. All analyses were done using CRAN R statistical software (2018-07-02, Feather Spray).

\section{(e) Ethical Note:}

This research was conducted in accordance with the guidelines set forth in the Louisiana State University IACUC Animal Care and Use Protocols \#10-115 and \#11-090. No humans were used in this study 


\section{Results}

(a) Influence of predation on schooling state:

Predation events had a negligible effect on the behavioral state of the school (MANOVA, $\mathrm{p}>0.35, \Lambda=0.966$, Table 1 , Figure 2 ), and did not significantly explain variability in our observations. The effects of predator attack were negligible when each metric of the schooling state was considered individually (Table 2). Pairwise comparisons between pre- and mid attack for each schooling metric exhibited no significant differences in school area (Tukey, $p>0.95$, Table 3), group speed (Tukey, $p>0.77$, Table 3), angular velocity (Tukey, $p>0.95$, Table 3), polarization (Tukey, $p>0.63$, Table 3), rotational order (Tukey, $\mathrm{p}>0.55$, Table 3), and correlation strength (Tukey, $\mathrm{p}>0.99$, Table 3 ), suggesting that schools largely stayed within the same range of behavioral variation prior to and during attack.

\section{(b) Influence of habitat context on schooling state:}

Habitat context significantly explained the variability in our observations (MANOVA, $p$ $<0.001, \Lambda=0.80071$, Table 1, Figure 2), particularly in schooling area occupied $\left(\right.$ ANOVA, $\left.F_{2,190}=6.935, \mathrm{p}=0.001\right)$, angular velocity $\left(\mathrm{ANOVA}, \mathrm{F}_{2,190}=9.34, \mathrm{p}<\right.$ 0.001), polarization (ANOVA, $\left.F_{2,190}=3.61, p=0.0288\right)$, rotational order (ANOVA, $F_{2}$, $190=3.98, \mathrm{p}=0.0203)$, and correlation strength $\left(\mathrm{ANOVA}, \mathrm{F}_{2,190}=6.06, \mathrm{p}=0.0028\right.$,

Table 3, Figure 2). In comparison to the free-field habitat, schools in the complex habitat occupied approximately $\sim 40 \%$ smaller area $(\mathrm{p}=0.034)$ and turned twice as slow $(\mathrm{p}=$ 0.0335). Schools in the marsh edge, compared to the free-field, occupied approximately $\sim 50 \%$ less area $(\mathrm{p}=0.003)$, and turned $\sim 2.5$ times slower $(\mathrm{p}<0.001)$. In addition, schools 
in the marsh edge were $\sim 30 \%$ more polarized ( $\mathrm{p}=0.025$ ), contained $\sim 30 \%$ higher rotational order $(\mathrm{p}=0.018)$, and exhibited $\mathrm{a} \sim 20 \%$ lower correlation strength $(\sim 0.14, \mathrm{p}=$ 0.002), than schools in the free-field. In comparing the complex habitat to the marsh edge, the same $\sim 20 \%$ correlation strength decrease pattern was found in comparison to the free-field. Since predation was not found to have a significant effect in explaining our observations, comparisons between habitats within the predation factor (pre/mid attack) did not produce any significant differences in schooling metrics and thus, behavioral schooling state.

\section{(c) Influence of predation and habitat context on schooling relationships:}

In the free-field habitat, predation had no effect on the inherent relationships between metrics in the schooling state. Variable loadings that were highly correlated were split into three uncorrelated groups: 1) polarization/rotational order, which loaded similarly on the first two principal components 2) area/correlation strength/angular velocity, which loaded strongly on the first principal component, and 3) speed, which loaded strongly on the second principal component (Figure 3, Table A1), and remained uncorrelated even under predator attack. In the marsh edge however, the three uncorrelated groups of variable loadings were affected by predator attack. In the marsh edge, speed remained uncorrelated with the other two groups (due to its strong loading on the third principal component, Table A1) prior to predator attack, but became more correlated with polarization and rotational order during attack (Figure 3). In contrast to both the free-field and marsh edge habitat contexts, group speed in the complex habitat context remained 
strongly loaded on the third principal component, yet exhibited higher correlation with polarization and rotational order both prior to, and during predator attack (Figure 3).

In comparison to the free-field, the relationships with the strongest coupling between the schooling metrics (polarization/rotational order, area/angular velocity/correlation strength) in the other habitats did not vary at all. The strongest correlations in the marsh edge and complex habitat contexts were equivalent to those in the free-field habitat context both before and during predator attack (Figure 3). However, habitat context did modulate other strong relationships found in the free-field.

Polarization and school area were negatively correlated (Figure 3,Table A1) prior to predator attack in the free-field habitat, but their correlation is no longer present in either the marsh edge or complex habitat contexts. In addition, the same effect was observed for the relationship between polarization/angular velocity, polarization/correlation strength, and rotational order/school area (Figure 3, Table A1). Each of these relationships became decoupled outside of the free-field habitat context, and were not influenced by predator attack.

\section{Discussion}

Our results demonstrate that habitat context had a much stronger effect on collective state of free-ranging fish schools than predation. Any type of habitat complexity, due to the presence of a boundary in the marsh edge or an obstacle in the complex habitat, induces changes to the behavioral schooling state. The larger occupied area in the free-field environment relative to the marsh edge and complex habitat contexts, supports previous evidence of larger shoal formation in structurally simple habitats (Orpwood et al., 2008). 
When comparing the impact of the marsh edge context on schooling state, the physical boundary edge appears to have a profound effect on schooling state relative to the free field. The observed decrease in school area, angular velocity, and correlation strength seem to be mitigated by a greater polarization and increased rotational order, where the presence of the physical boundary elicits tighter schooling and directional alignment parallel to the boundary, at the possible cost of a decrease in information transfer rate. This evidence is in line with the effect of boundaries on driving transitions of schooling state discovered in controlled laboratory experiments (Tunstrøm et al., 2013).

Additionally, the presence of the physical boundary constrains the geometry of the school and creates a spatial upper bound on the area that the school can occupy, supported by the decrease in school area and angular velocity. This may be attributed to the safety provided by restricting the direction from which predators may attack, affecting the potential mechanics of predator attack and possibly leading to changes in school vigilance (Higham et al., 2015).

In the complex habitat, the differences in area, and angular velocity compared to the free-field suggest that the schooling state is being modulated by the presence of obstacles in the environment. The types of structures observed in these environments are uniformly solid and unable to be used as a point of safety for individuals in the school, suggesting that the structures act more like obstacles than areas of refuge. The decrease in area and angular velocity suggests that schools are adopting tighter formations, typical of schools exposed to high predation risk (Magurran and Pitcher, 1987; Orpwood et al., 2008). This may be due to heightened vigilance, derived from the difference in sensory environment compared to a free-field context, requiring the ability to account for any 
potential obstacles the school may encounter while behaving within the habitat. In these complex environments, the sensory field can confound predator detection by the school via external visual information (in the form of rocks/pilings/traps) that acts as a secondary stimulus or source of noise to the visual information of the impending predator attack. The presence of this type of confounding information, in conjunction with a stimulus that requires a behavioral response suggests that local environment plays a large role in structuring collective behavior to predator attack.

Our results also indicate that there are few differences in schooling state between the habitats with any type of complexity in the environment. This is likely a reflection of the similarity of the habitat contexts. The presence of a boundary or obstacle modulate the schooling state in a similar manner, however further studies are needed to determine if habitats with high spatial heterogeneity or anthropogenically-influenced habitats (e.g. water control structures) differ drastically in their effect on collective behavior.

In contrast to habitat context, behavioral schooling state in fish schools appear to be robust to predator attack, which implies that there exists a plasticity in the schooling behaviors that can account for the sudden presence of external stimuli. More importantly, this pattern is conserved within habitat context, since no differences were found in schooling state before and during predator attack in both the marsh edge and complex habitat contexts. Predation pressure was largely equivalent across all habitat contexts (based on catch ratios of predator to prey abundance) (Klotzbach, 2013), suggesting that prey schools were not influenced by environmental adaptation to the inherent risk level (Ioannou et al., 2017). Habitat complexity typically decreases predation due to the increase in refugia (Crowder and Cooper, 1982; Holbrook and Schmitt, 2002), however, 
the lack of differences in the schooling state response before and after predator attack even in the marsh edge and complex habitats suggest that local environment plays a much larger role in structuring collective behavior than predation.

When examining the relationships between the schooling state metrics, our results indicate that changes in the presence and strength of correlation provide further insight into whether predation and environment modulates group behavior of fish schools. In the free-field, we see no changes in the relationships between schooling metrics during predator attack, but find that the marsh edge and complex habitat contexts influence the relationship between group speed and orientational polarization/rotational order. The appearance of coupling between speed and polarization/rotational order during predator attack in the marsh edge is consistent with the idea that the edge boundary affects the school state, promoting alignment with the boundary as the school is quickly escaping from predators. The tighter coupling between speed and polarization of schools when attacked by predators is consistent with prior investigations into the mechanisms controlling the collective response (Calovi et al., 2014; Lecheval et al., 2018; Tunstrøm et al., 2013; Viscido et al., 2004). This further supports the premise that the local environment (such as the presence of an obstacle) strongly influences collective patterns and anti-predator schooling behavior.

When comparing schooling state relationships within environmental contexts, we found a combination of robustness as well as modulation in the schooling state. In the free-field condition, the strongest schooling metric relationships (rotational order/polarization, area/angular velocity, correlation strength/angular velocity, area/correlation strength), remain consistent regardless of predator attack. This provides 
further evidence to support the idea that the anti-predator response falls within the range of behavioral plasticity. However, these relationships are conserved in both the marsh edge and complex habitat contexts, implying that certain aspects of the collective behavior are inherently robust to any type of outside sensory stimuli perceived by the school.

As a complement to robust relationships found across all habitat types, the fact that some relationships decouple outside of the free-field suggests that the schooling state in the marsh edge and complex habitats is altered directly by the habitat context itself. The loss of negatively correlated relationships (school area/polarization, polarization/angular velocity, school area/rotational order, correlation strength/polarization) in both the marsh edge and complex habitat context show that the physical differences in the environment directly modulate collective behavior. The habitat context, in this case, seems to influence the tradeoff between area and polarization/rotational order, as well as polarization and correlation strength/angular velocity; the loss of this tradeoff may allow looser schooling without loss of directional and rotational coherence, as well as information transfer. This is in contrast to the effect of other environmental factors, such as turbidity (Chamberlain and Ioannou, 2019) and anthropogenic noise (Herbert-Read et al., 2017b), which act to weaken school structure and responses. The purpose and mechanism underlying habitat influences on collective behavior remains unclear, and our results call for further the investigation of the functional explanations of changes in collective state of fish schools at the face of habitat complexity in natural settings. 
In this study, we examined the influence of habitat context on the state and structure of freely behaving fish schools before and during predator attack. We found that in general, habitat context, not predation, cause changes in schooling state relative to a free-field environment. The impact of the type of habitat context affects the school state, where the presence of a boundary or finite-sized obstacles causes direct changes in schooling state and the relationships between them. These results demonstrate, in concert with studies of other environmental factors, that the surrounding environmental context, regardless of predation, is largely influential in the modulation of collective behavior in fish schools. In the face of global climate change, habitat modification and potential destruction may then influence movement, foraging, and local behavior patterns of large groups of prey fish of critical and commercial importance. Knowledge of the environmental impacts on the behavioral dynamics of these animal aggregations will help refine our understanding of the patterns of collective behavior.

\section{References}

Abrahams, M. V. and Kattenfeld, M. G. (1997). The role of turbidity as a constraint on predator-prey interactions in aquatic environments. Behavioral Ecology and Sociobiology 40, 169-174.

Ajemian, M. J., Sohel, S. and Mattila, J. (2015). Effects of turbidity and habitat complexity on antipredator behavior of three-spined sticklebacks (Gasterosteus aculeatus): Antipredator behavior in sticklebacks. Environmental Biology of Fishes 98, 45-55.

Attanasi, A., Cavagna, A., Del Castello, L., Giardina, I., Melillo, S., Parisi, L., Pohl, O., Rossaro, B., Shen, E., Silvestri, E., et al. (2014a). Collective Behaviour without Collective Order in Wild Swarms of Midges. PLoS Computational Biology 10,

Attanasi, A., Cavagna, A., Del Castello, L., Giardina, I., Melillo, S., Parisi, L., Pohl, O., Rossaro, B., Shen, E., Silvestri, E., et al. (2014b). Collective Behaviour without 
Collective Order in Wild Swarms of Midges. PLoS Computational Biology 10, e1003697.

Attanasi, A., Cavagna, A., Del Castello, L., Giardina, I., Grigera, T., Jelić, A., Melillo, S., Parisi, L., Pohl, O., Shen, E., et al. (2014c). Information transfer and behavioural inertia in starling flocks. Nature Physics 10, 691-696.

Berdahl, A., Torney, C. J., Ioannou, C. C., Faria, J. J. and Couzin, I. D. (2013). Emergent sensing of complex environments by mobile animal groups. Science 339, 574576.

Borner, K. K., Krause, S., Mehner, T., Uusi-Heikkilä, S., Ramnarine, I. W. and Krause, J. (2015). Turbidity affects social dynamics in Trinidadian guppies. Behavioral Ecology and Sociobiology 69, 645-651.

Boswell, K. M., Miller, M. W. and Wilson, C. A. (2007). A lightweight transducer platform for use in stationary shallow water horizontal-aspect acoustic surveys. Fisheries Research 85, 291-294.

Boswell, K. M., Kimball, M. E., Rieucau, G., Martin, J. G. A., Jacques, D. A., Correa, D. and Allen, D. M. (2019). Tidal Stage Mediates Periodic Asynchrony Between Predator and Prey Nekton in Salt Marsh Creeks. Estuaries and Coasts 42, 13421352.

Brown, G. E., Rive, A. C., Ferrari, M. C. O. and Chivers, D. P. (2006). The dynamic nature of antipredator behavior: prey fish integrate threat-sensitive antipredator responses within background levels of predation risk. Behavioral Ecology and Sociobiology 61, 9-16.

Calovi, D. S., Lopez, U., Ngo, S., Sire, C., Chaté, H. and Theraulaz, G. (2014). Swarming, schooling, milling: phase diagram of a data-driven fish school model. New J. Phys. 16, 015026.

Cavagna, A., Cimarelli, A., Giardina, I., Orlandi, A., Parisi, G., Procaccini, A., Santagati, R. and Stefanini, F. (2008). New statistical tools for analyzing the structure of animal groups. Mathematical Biosciences 214, 32-37.

Cavagna, A., Cimarelli, A., Giardina, I., Parisi, G., Santagati, R., Stefanini, F. and Viale, M. (2010). Scale-free correlations in starling flocks. Proceedings of the National Academy of Sciences 107, 11865-11870.

Cavagna, A., Del Castello, L., Giardina, I., Grigera, T., Jelic, A., Melillo, S., Mora, T., Parisi, L., Silvestri, E. and Viale, M. (2015). Flocking and turning: a new model for self-organized collective motion. Journal of Statistical Physics 158, 601-627. 
Chamberlain, A. C. and Ioannou, C. C. (2019). Turbidity increases risk perception but constrains collective behaviour during foraging by fish shoals. Animal Behaviour $156,129-138$.

Cook, W. L. and Streams, F. A. (1984). Fish predation on Notonecta (Hemiptera): relationship between prey risk and habitat utilization. Oecologia 64, 177-183.

Couzin, I. D., Krause, J., James, R., Ruxton, G. D. and Franks, N. R. (2002). Collective memory and spatial sorting in animal groups. Journal of theoretical biology 218, $1-11$.

Couzin, I. D., James, R., Mawdsley, D., Croft, D. P. and Krause, J. (2006). Social organization and information transfer in schooling fishes. Fish cognition and behavior 166-185.

Crowder, L. B. and Cooper, W. E. (1982). Habitat Structural Complexity and the Interaction Between Bluegills and Their Prey. Ecology 63, 1802.

De Robertis, A., Ryer, C. H., Veloza, A. and Brodeur, R. D. (2003). Differential effects of turbidity on prey consumption of piscivorous and planktivorous fish. Canadian Journal of Fisheries and Aquatic Sciences 60, 1517-1526.

Delcourt, J. and Poncin, P. (2012). Shoals and schools: back to the heuristic definitions and quantitative references. Reviews in Fish Biology and Fisheries 22, 595-619.

Domenici, P. and Hale, M. E. (2019). Escape responses of fish: a review of the diversity in motor control, kinematics and behaviour. J Exp Biol 222,.

Domenici, P., Lefrancois, C. and Shingles, A. (2007). Hypoxia and the antipredator behaviours of fishes. Philosophical Transactions of the Royal Society B:

Biological Sciences 362, 2105-2121.

Domenici, P., Steffensen, J. F. and Marras, S. (2017). The effect of hypoxia on fish schooling. Phil. Trans. R. Soc. B 372, 20160236.

Figueiredo, B. R. S., Mormul, R. P. and Benedito, E. (2015). Structural complexity and turbidity do not interact to influence predation rate and prey selectivity by a small visually feeding fish. Marine and Freshwater Research 66, 170.

Fleet, D. and Weiss, Y. (2006). Optical flow estimation. In Handbook of mathematical models in computer vision, pp. 237-257. Springer.

Garner, S. B. (2012). Effects of mimic artificial oyster reefs on the ecology of juvenile fishes in marsh ponds: a before-after-control-impact analysis. LSU Master's Theses. 
Gautrais, J., Ginelli, F., Fournier, R., Blanco, S., Soria, M., Chaté, H. and Theraulaz, G. (2012). Deciphering Interactions in Moving Animal Groups. PLoS Computational Biology 8, e1002678.

Handegard, N. O., Boswell, K. M., Ioannou, C. C., Leblanc, S. P., Tjøstheim, D. B. and Couzin, I. D. (2012). The Dynamics of Coordinated Group Hunting and Collective Information Transfer among Schooling Prey. Current Biology 22, 1213-1217.

Herbert-Read, J. E., Buhl, J., Hu, F., Ward, A. J. and Sumpter, D. J. (2015). Initiation and spread of escape waves within animal groups. Royal Society open science 2, 140355 .

Herbert-Read, J. E., Rosén, E., Szorkovszky, A., Ioannou, C. C., Rogell, B., Perna, A., Ramnarine, I. W., Kotrschal, A., Kolm, N., Krause, J., et al. (2017a). How predation shapes the social interaction rules of shoaling fish. Proc. R. Soc. B 284, 20171126.

Herbert-Read, J. E., Kremer, L., Bruintjes, R., Radford, A. N. and Ioannou, C. C. (2017b). Anthropogenic noise pollution from pile-driving disrupts the structure and dynamics of fish shoals. Proc. R. Soc. B 284, 20171627.

Higham, T. E., Stewart, W. J. and Wainwright, P. C. (2015). Turbulence, Temperature, and Turbidity: The Ecomechanics of Predator-Prey Interactions in Fishes. Integrative and Comparative Biology 55, 6-20.

Hoare, D. J., Couzin, I. D., Godin, J.-G. J. and Krause, J. (2004). Context-dependent group size choice in fish. Animal Behaviour 67, 155-164.

Holbrook, S. J. and Schmitt, R. J. (2002). Competition for Shelter Space Causes DensityDependent Predation Mortality in Damselfishes. Ecology 83, 2855-2868.

Ioannou, C. C. (2017). Grouping and Predation. In Encyclopedia of Evolutionary Psychological Science (ed. Shackelford, T. K.) and Weekes-Shackelford, V. A.), pp. 1-6. Cham: Springer International Publishing.

Ioannou, C. C., Bartumeus, F., Krause, J. and Ruxton, G. D. (2011). Unified effects of aggregation reveal larger prey groups take longer to find. Proc. R. Soc. B 278, 2985-2990.

Ioannou, C. C., Guttal, V. and Couzin, I. D. (2012). Predatory Fish Select for Coordinated Collective Motion in Virtual Prey. Science 337, 1212-1215.

Ioannou, C. C., Ramnarine, I. W. and Torney, C. J. (2017). High-predation habitats affect the social dynamics of collective exploration in a shoaling fish. Science Advances 3, e1602682. 
Jeschke, J. M. and Tollrian, R. (2007). Prey swarming: which predators become confused and why? Animal Behaviour 74, 387-393.

Kent, M. I. A., Lukeman, R., Lizier, J. T. and Ward, A. J. W. (2019). Speed-mediated properties of schooling. R. Soc. open sci. 6, 181482.

Kimbell, H. S. and Morrell, L. J. (2015). Turbidity influences individual and group level responses to predation in guppies, Poecilia reticulata. Animal Behaviour 103, $179-185$.

King, A. J., Fehlmann, G., Biro, D., Ward, A. J. and Fürtbauer, I. (2018). Re-wilding Collective Behaviour: An Ecological Perspective. Trends in ecology \& evolution.

Klotzbach, K. E. (2013). Impacts of artificial reef addition on the nekton community of Louisiana marsh ponds: a before-after-control-impact analysis.

Krause, J. and Ruxton, G. D. (2002). Living in Groups. Oxford University Press.

Lecheval, V., Jiang, L., Tichit, P., Sire, C., Hemelrijk, C. K. and Theraulaz, G. (2018). Social conformity and propagation of information in collective U-turns of fish schools. Proc. R. Soc. B 285, 20180251.

Lichtenstein, J. L. L., Daniel, K. A., Wong, J. B., Wright, C. M., Doering, G. N., CostaPereira, R. and Pruitt, J. N. (2019). Habitat structure changes the relationships between predator behavior, prey behavior, and prey survival rates. Oecologia 190, 297-308.

Lopez, U., Gautrais, J., Couzin, I. D. and Theraulaz, G. (2012). From behavioural analyses to models of collective motion in fish schools. Interface Focus 2, $693-$ 707.

Magurran, A. E. and Higham, A. (1988). Information transfer across fish shoals under predator threat. Ethology 78, 153-158.

Magurran, A. E. and Pitcher, T. J. (1987). Provenance, shoal size and the sociobiology of predator-evasion behaviour in minnow shoals. Proc. R. Soc. Lond. B 229, 439465 .

Orpwood, J. E., Magurran, A. E., Armstrong, J. D. and Griffiths, S. W. (2008). Minnows and the selfish herd: effects of predation risk on shoaling behaviour are dependent on habitat complexity. Animal Behaviour 76, 143-152.

Otsu, N. (1979). A threshold selection method from gray-level histograms. IEEE transactions on systems, man, and cybernetics 9, 62-66.

Parrish, J. K. (1989). Re-examining the selfish herd: are central fish safer? Animal Behaviour 38, 1048-1053. 
Parrish, J. K., Viscido, S. V. and Grunbaum, D. (2002). Self-organized fish schools: an examination of emergent properties. The biological bulletin 202, 296-305.

Partridge, B. L., Pitcher, T., Cullen, J. M. and Wilson, J. (1980). The three-dimensional structure of fish schools. Behavioral Ecology and Sociobiology 6, 277-288.

Pitcher, T. J. (1983). Heuristic definitions of fish shoaling behaviour. Animal Behaviour.

Radakov, D. V. (1973). Schooling in the ecology of fish.

Rieucau, G., Boswell, K. M., De Robertis, A., Macaulay, G. J. and Handegard, N. O. (2014). Experimental Evidence of Threat-Sensitive Collective Avoidance Responses in a Large Wild-Caught Herring School. PLoS ONE 9, e86726.

Rieucau, G., Fernö, A., Ioannou, C. C. and Handegard, N. O. (2015). Towards of a firmer explanation of large shoal formation, maintenance and collective reactions in marine fish. Reviews in Fish Biology and Fisheries 25, 21-37.

Rieucau, G., Holmin, A. J., Castillo, J. C., Couzin, I. D. and Handegard, N. O. (2016a). School level structural and dynamic adjustments to risk promote information transfer and collective evasion in herring. Animal Behaviour 117, 69-78.

Rieucau, G., Holmin, A. J., Castillo, J. C., Couzin, I. D. and Handegard, N. O. (2016b). School level structural and dynamic adjustments to risk promote information transfer and collective evasion in herring. Animal Behaviour 117, 69-78.

Rosenthal, S. B., Twomey, C. R., Hartnett, A. T., Wu, H. S. and Couzin, I. D. (2015). Revealing the hidden networks of interaction in mobile animal groups allows prediction of complex behavioral contagion. Proceedings of the National Academy of Sciences 112, 4690-4695.

Ruxton, G. D., Jackson, A. L. and Tosh, C. R. (2007). Confusion of predators does not rely on specialist coordinated behavior. Behavioral Ecology 18, 590-596.

Ryer, C. and Olla, B. 1. (1998). Effect of light on juvenile walleye pollock shoaling and their interaction with predators. Mar. Ecol. Prog. Ser. 167, 215-226.

Strandburg-Peshkin, A., Twomey, C. R., Bode, N. W., Kao, A. B., Katz, Y., Ioannou, C. C., Rosenthal, S. B., Torney, C. J., Wu, H. S., Levin, S. A., et al. (2013). Visual sensory networks and effective information transfer in animal groups. Current Biology 23, R709-R711.

Tunstrøm, K., Katz, Y., Ioannou, C. C., Huepe, C., Lutz, M. J. and Couzin, I. D. (2013). Collective States, Multistability and Transitional Behavior in Schooling Fish. PLoS Computational Biology 9, e1002915. 
Turner, G. F. and Pitcher, T. J. (1986). Attack Abatement: A Model for Group Protection by Combined Avoidance and Dilution. The American Naturalist 128, 228-240.

Viscido, S. V., Parrish, J. K. and Grünbaum, D. (2004). Individual behavior and emergent properties of fish schools: a comparison of observation and theory. Marine Ecology Progress Series 273, 239-250.

Ward, A. J. W., Herbert-Read, J. E., Sumpter, D. J. T. and Krause, J. (2011). Fast and accurate decisions through collective vigilance in fish shoals. Proceedings of the National Academy of Sciences 108, E27-E27.

Weetman, D., Atkinson, D. and Chubb, J. C. (1998). Effects of temperature on antipredator behaviour in the guppy, Poecilia reticulata. Animal behaviour 55, 13611372.

Zienkiewicz, A. K., Ladu, F., Barton, D. A. W., Porfiri, M. and Bernardo, M. D. (2018). Data-driven modelling of social forces and collective behaviour in zebrafish. Journal of Theoretical Biology 443, 39-51. 
Figures

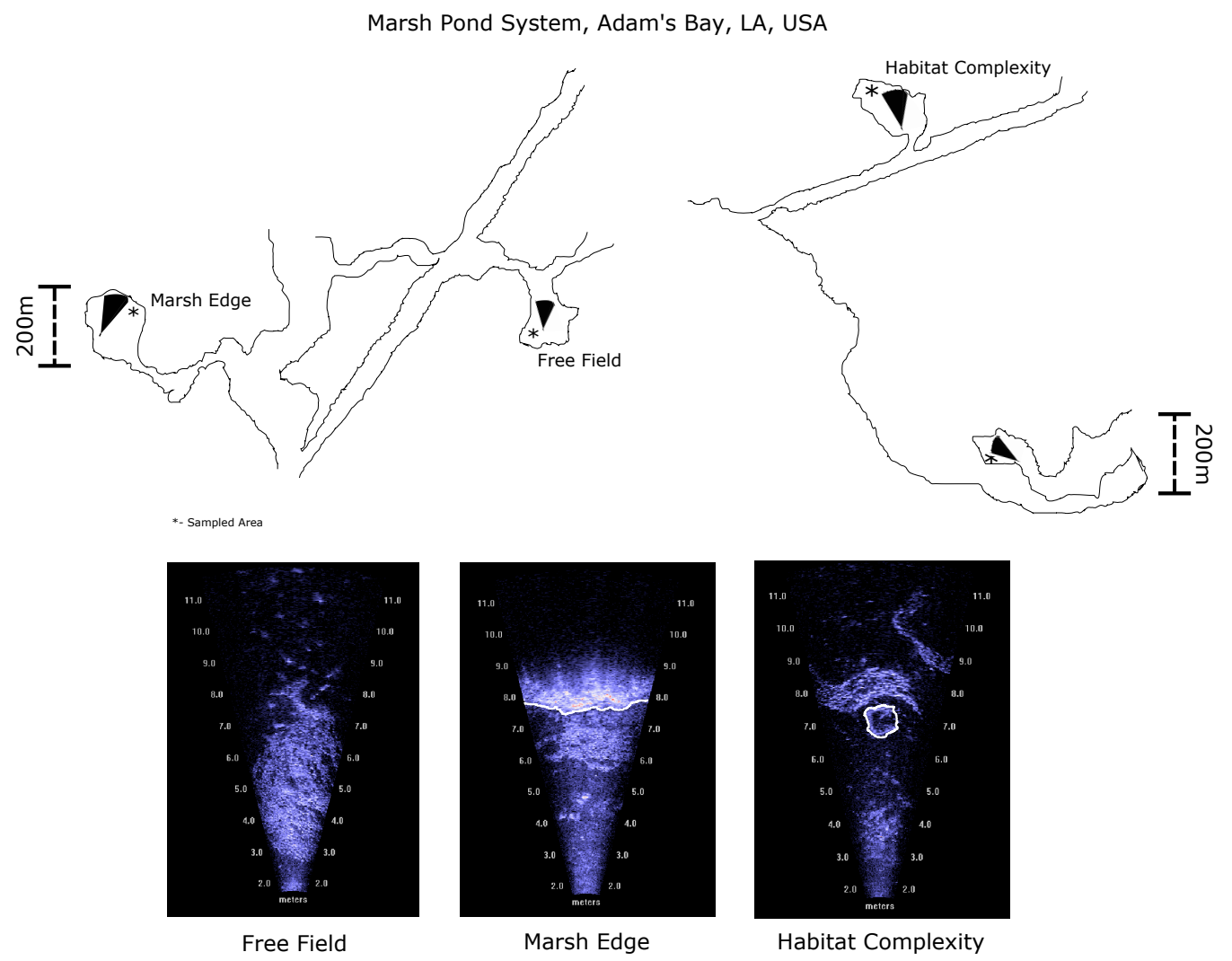

Figure (1): Illustration of marsh pond sample area in Louisiana, USA. Three sonar cone positions represent the three habitat conditions surveyed. Each surveyed condition across the pond was at a shallow depth $(<2 \mathrm{~m})$, with no significant tidal currents present. The freefield condition contained a sediment bottom, free of structure and obstacles. The marsh edge condition is equivalent to the free-field condition with the exception of an edge boundary (outlined in white) that impedes movement of swimming organisms. The habitat complexity condition was comprised of a sediment bottom, but also contained rocks, wood pilings, crab traps, and detritus that served as obstacles toward movement (outlined in white). The habitat complexity condition did not contain an edge boundary so each direction of movement was preserved. 


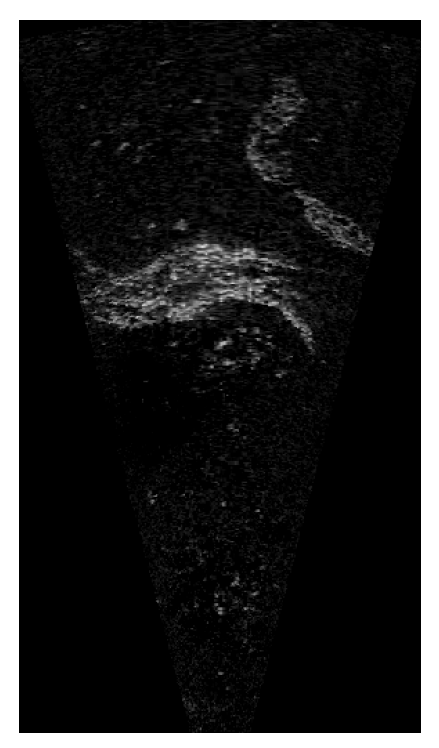

Figure (M1): Filtered acoustic image with denoising and background subtraction. Denoising was performed using wavelet-based shrinkage methods; background estimates for each behavioral interaction used the $30^{\text {th }}$ percentile for each pixel intensity across all frames. Once each background is estimated, it is subtracted from each denoised frame in the behavioral interaction.

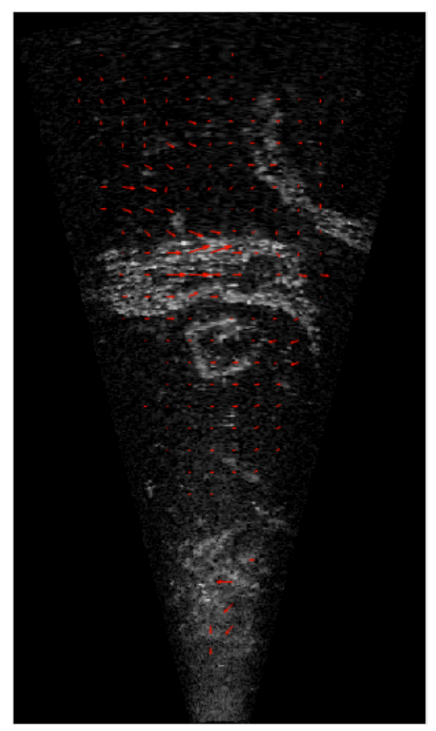

Figure (M2): Particle Image Velocimetry (PIV) computed over a 32x32 pixel window to extract velocity estimates for each frame in the behavioral interaction. PIV was computed for the entire image, using changes in image intensity to estimate velocity magnitude and direction. PIV vectors were filtered temporally over five frame intervals, and further filtered spatially using a $3 \times 3$ pixel window median filter. 


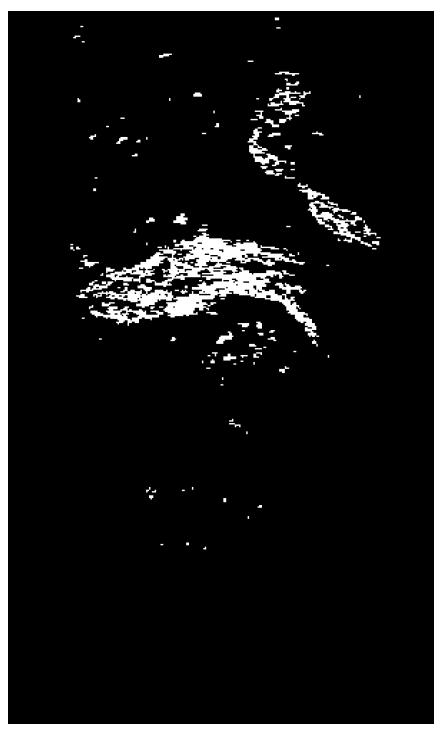

Figure (M3): Image filtering using Otsu's method and pixel area-based morphological filtering of small objects to isolate fish schools in each acoustic image. The processed image retains the main fish schools in the image and is ready for further processing to extract the boundary of the school of interest.

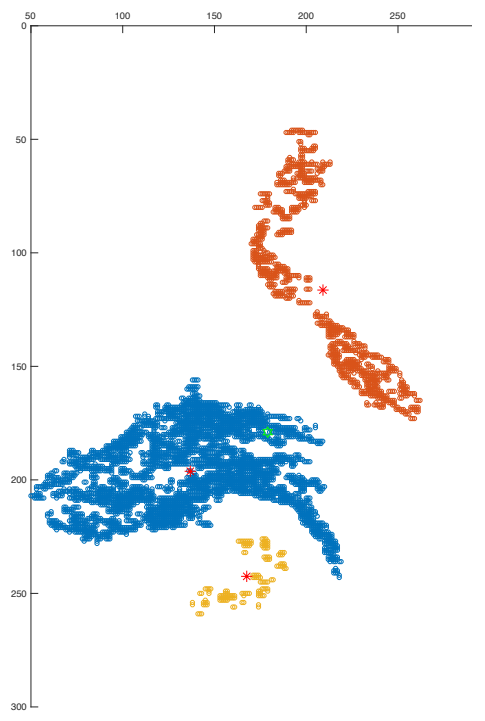

Figure (M4): School regions isolated by segmenting each acoustic image using cluster analysis to distinguish fish schools. The processed image was converted to a two dimensional point cloud, and schools were identified using Euclidean distance (L2 norm) clustering at a distance of $0.3 \mathrm{~m}$. Individual schools detected are denoted by color, with red asterisks representing each schools' center of mass. 

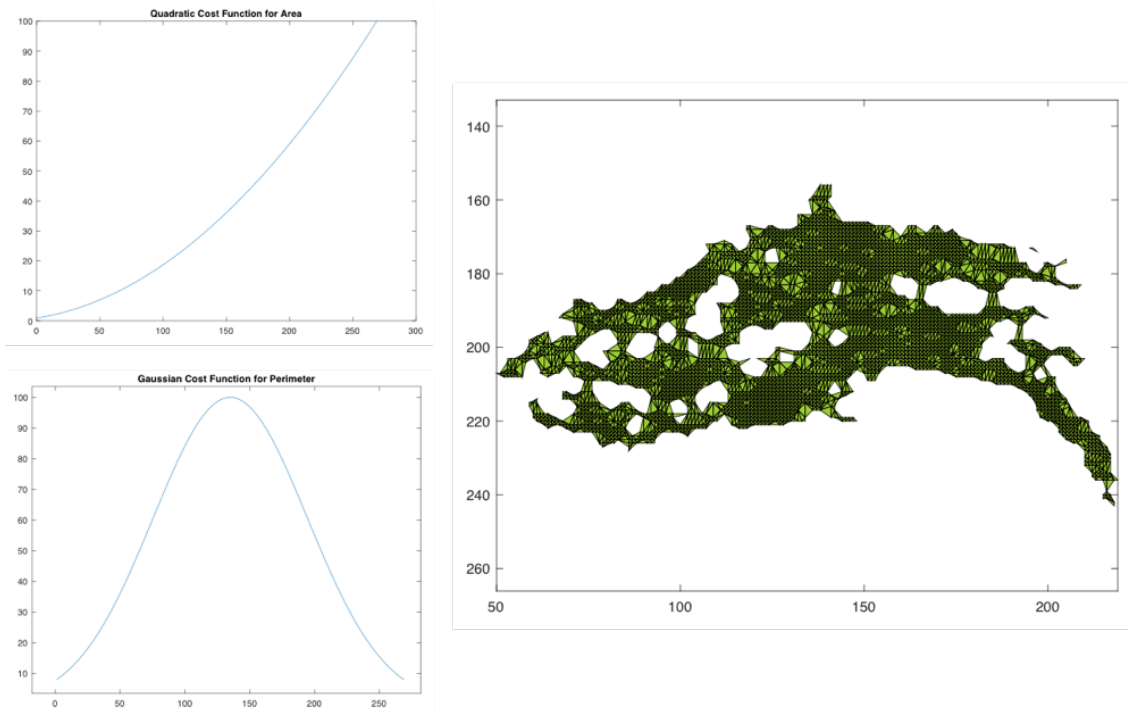

Figure (M5): Boundary optimization of detected school cluster with associated cost functions for area and perimeter. The detected school of interest was registered using alpha shapes filtering, and cost functions ascribed to area and perimeter were used to derive the optimum value for the alpha radius.

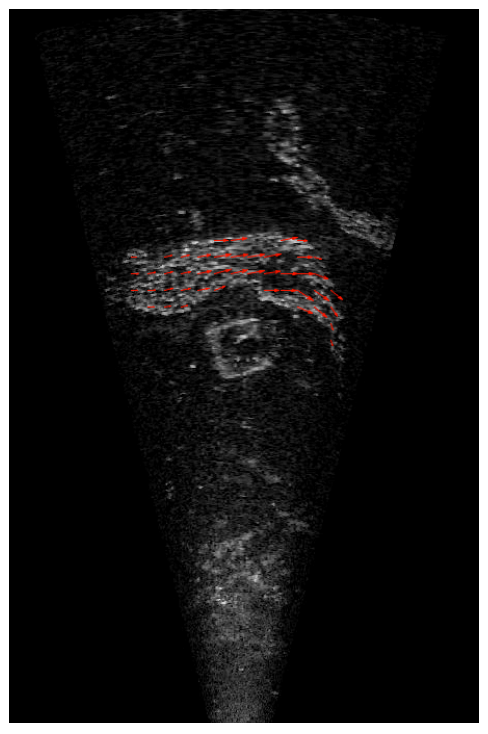

Figure (M6): Final detected school boundary with representative PIV velocity estimates used for analysis of schooling state. The optimized alpha shape school boundary was used to filter the PIV velocity field to include vectors located within the school, providing an accurate estimate of individual behavior within the school. Each school was tracked frame by frame via a Kalman filter over the course of the behavioral interaction. 

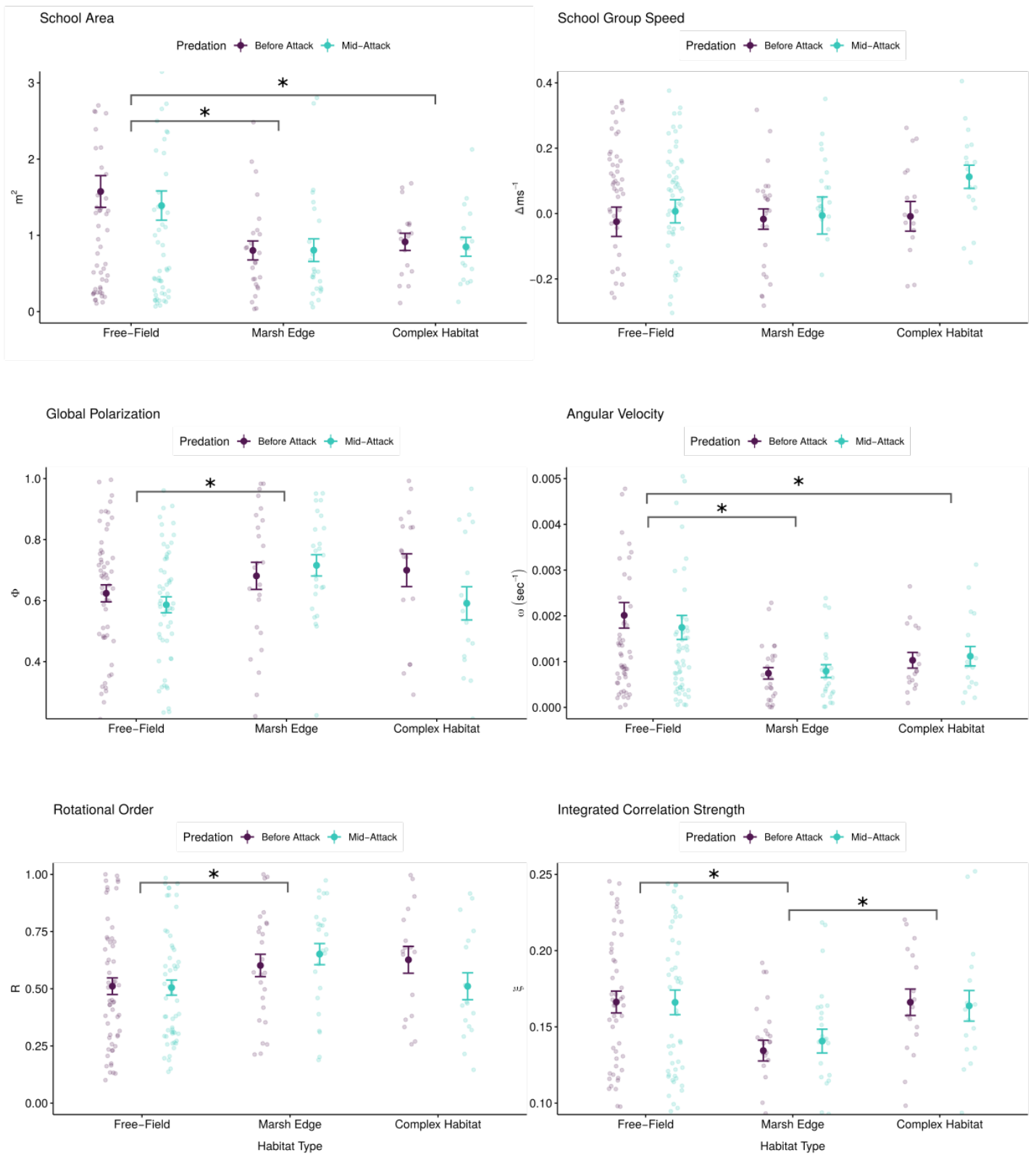

Figure (2): Comparison of behavioral schooling metrics before and during predator attack over an environmental gradient. All conditions pre-, and during predator attack are reported for each habitat context, and are color coded within each habitat type. Results show mean values of each schooling state metric, with data distributions represented underneath. Error bars were determined via the standard error, and significant differences between habitat contexts are denoted by the asterisk. Six schooling state parameters were quantified, prior to, and during predator attack, across three environmental contexts, for a total of 36 unique measurements. 

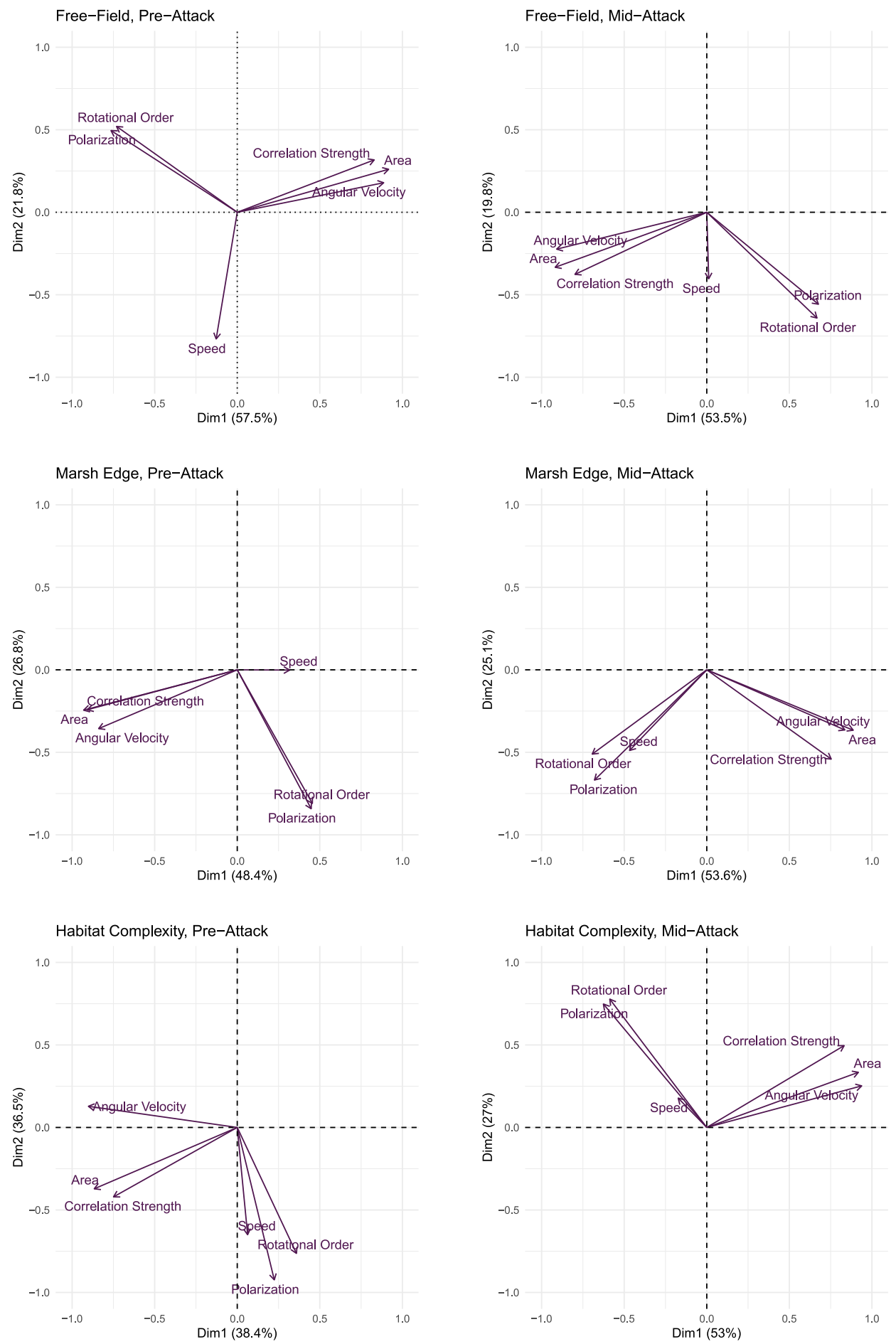

Figure (3): PCA variable loading plots for each habitat context and predation status condition. PCA was scaled to unit variance, with no rotation. The two largest principal components (all captured $>75 \%$ of the variability) were represented on the $\mathrm{x}$ and $\mathrm{y}$ axes. The percent of the variability explained by each principal component is denoted next to the name. 


\begin{tabular}{lrrrrr}
\hline & \multicolumn{5}{c}{ MANOVA } \\
\cline { 2 - 6 } & df & Wilks & num df & den df & p-value \\
\hline Predation & 1 & 0.96630 & 6 & 185 & 0.37881 \\
Habitat & 2 & 0.80071 & 12 & 370 & 0.00004 \\
Predation:Habitat & 2 & 0.97095 & 12 & 370 & 0.93800 \\
Residuals & 190 & & & & \\
\hline
\end{tabular}

Table (1): Two-way MANOVA table comparing the effect of Predation, Habitat, and their interaction among the six schooling state variables.

\begin{tabular}{lrrrr}
\hline & \multicolumn{4}{c}{ School Area } \\
\cline { 2 - 5 } & df & SS & F & p-value \\
\hline Predation & 1 & 0.6596 & 0.4521 & 0.5022 \\
Habitat & 2 & 20.2378 & 6.9351 & 0.0012 \\
Predation:Habitat & 2 & 0.3331 & 0.1141 & 0.8922 \\
Residuals & 190 & 277.2271 & & \\
& \multicolumn{5}{c}{ Angular Velocity } \\
\cline { 2 - 5 } & df & SS & F & p-value \\
\hline Predation & 1 & 0.000001 & 0.286311 & 0.593220 \\
Habitat & 2 & 0.000049 & 9.340087 & 0.000135 \\
Predation:Habitat & 2 & 0.000001 & 0.251675 & 0.777756 \\
Residuals & 190 & 0.000495 & & \\
& \multicolumn{5}{c}{ Rotational Order } \\
\cline { 2 - 6 } & df & SS & F & p-value \\
\hline Predation & 1 & 0.0058 & 0.0925 & 0.7613 \\
Habitat & 2 & 0.5023 & 3.9803 & 0.0203 \\
Predation:Habitat & 2 & 0.1400 & 1.1093 & 0.3319 \\
Residuals & 190 & 11.9876 & & \\
\hline
\end{tabular}

\begin{tabular}{lrrrr}
\hline & \multicolumn{4}{c}{ Group Speed } \\
\cline { 2 - 5 } & $\mathrm{df}$ & $\mathrm{SS}$ & $\mathrm{F}$ & p-value \\
\hline Predation & 1 & 0.0867 & 1.2196 & 0.2708 \\
Habitat & 2 & 0.1077 & 0.7571 & 0.4705 \\
Predation:Habitat & 2 & 0.0679 & 0.4775 & 0.6211 \\
Residuals & 190 & 13.5102 & & \\
& \multicolumn{4}{c}{ Polarization } \\
\cline { 2 - 5 } & $\mathrm{df}$ & $\mathrm{SS}$ & $\mathrm{F}$ & $\mathrm{p}$-value \\
\hline Predation & 1 & 0.0485 & 1.1529 & 0.2843 \\
Habitat & 2 & 0.3044 & 3.6148 & 0.0288 \\
Predation:Habitat & 2 & 0.1061 & 1.2596 & 0.2861 \\
Residuals & 190 & 7.9990 & & \\
& \multicolumn{5}{c}{ Correlation Strength } \\
\cline { 2 - 5 } & $\mathrm{df}$ & SS & $\mathrm{F}$ & p-value \\
\hline Predation & 1 & 0.0001 & 0.0221 & 0.8819 \\
Habitat & 2 & 0.0300 & 6.0604 & 0.0028 \\
Predation:Habitat & 2 & 0.0005 & 0.0969 & 0.9077 \\
Residuals & 190 & 0.4698 & & \\
\hline
\end{tabular}

Table (2): Univariate ANOVA tables for each schooling state metric, comparing effects of Predation, Habitat, and interaction. 


\begin{tabular}{|c|c|}
\hline \multicolumn{2}{|l|}{ School Area } \\
\hline Treatment Comparison & P-value \\
\hline \multicolumn{2}{|l|}{ Habitat } \\
\hline Free-Field:Habitat Complexity & 0.034 \\
\hline Marsh Edge:Habitat Complexity & 1.000 \\
\hline Free-Field:Marsh Edge & 0.003 \\
\hline \multicolumn{2}{|l|}{ Pre-/Mid-Predator Attack } \\
\hline Free-Field & 0.965 \\
\hline Marsh Edge & 1.000 \\
\hline Habitat Complexity & 1.000 \\
\hline \multicolumn{2}{|l|}{ Pre-Attack } \\
\hline Free-Field:Marsh Edge & 0.088 \\
\hline Free-Field:Habitat Complexity & 0.361 \\
\hline Marsh Edge:Habitat Complexity & 1.000 \\
\hline \multicolumn{2}{|l|}{ Mid-Attack } \\
\hline Free-Field:Marsh Edge & 0.338 \\
\hline Free-Field:Habitat Complexity & 0.589 \\
\hline Marsh Edge:Habitat Complexity & 1.000 \\
\hline \multicolumn{2}{|l|}{ Angular Velocity } \\
\hline Treatment Comparison & P-value \\
\hline \multicolumn{2}{|l|}{ Habitat } \\
\hline Free-Field:Habitat Complexity & 0.0335 \\
\hline Marsh Edge:Habitat Complexity & 1.0000 \\
\hline Free-Field:Marsh Edge & 0.0002 \\
\hline \multicolumn{2}{|l|}{ Pre-/Mid-Predator Attack } \\
\hline Free-Field & 0.9538 \\
\hline Marsh Edge & 1.0000 \\
\hline Habitat Complexity & 1.0000 \\
\hline \multicolumn{2}{|l|}{ Pre-Attack } \\
\hline Free-Field:Marsh Edge & 0.0162 \\
\hline Free-Field:Habitat Complexity & 0.2441 \\
\hline Marsh Edge:Habitat Complexity & 0.9932 \\
\hline \multicolumn{2}{|l|}{ Mid-Attack } \\
\hline Free-Field:Marsh Edge & 0.1422 \\
\hline Free-Field:Habitat Complexity & 0.7234 \\
\hline Marsh Edge:Habitat Complexity & 0.9876 \\
\hline \multicolumn{2}{|l|}{ Rotational Order } \\
\hline Treatment Comparison & $\mathrm{P}$-value \\
\hline \multicolumn{2}{|l|}{ Habitat } \\
\hline Free-Field:Habitat Complexity & 0.654 \\
\hline Marsh Edge:Habitat Complexity & 0.900 \\
\hline Free-Field:Marsh Edge & 0.018 \\
\hline \multicolumn{2}{|l|}{ Pre-/Mid-Predator Attack } \\
\hline Free-Field & 1.000 \\
\hline Marsh Edge & 0.982 \\
\hline Habitat Complexity & 0.760 \\
\hline \multicolumn{2}{|l|}{ Pre-Attack } \\
\hline Free-Field:Marsh Edge & 0.664 \\
\hline Free-Field:Habitat Complexity & 0.559 \\
\hline Marsh Edge:Habitat Complexity & 1.000 \\
\hline \multicolumn{2}{|l|}{ Mid-Attack } \\
\hline Free-Field:Marsh Edge & 0.153 \\
\hline Free-Field:Habitat Complexity & 1.000 \\
\hline Marsh Edge:Habitat Complexity & 0.481 \\
\hline
\end{tabular}

\begin{tabular}{lc}
\hline \multicolumn{2}{c}{ Group Speed } \\
\cline { 1 - 1 } Treatment Comparison & \\
\cline { 1 - 1 } Habitat & \\
Free-Field:Habitat Complexity & 0.726 \\
Marsh Edge:Habitat Complexity & 0.855 \\
Free-Field:Marsh Edge & 1.000 \\
Pre-/Mid-Predator Attack & \\
Free-Field & 0.988 \\
Marsh Edge & 1.000 \\
Habitat Complexity & 0.772 \\
Pre-Attack & \\
Free-Field:Marsh Edge & 1.000 \\
Free-Field:Habitat Complexity & 1.000 \\
Marsh Edge:Habitat Complexity & 1.000 \\
Mid-Attack & \\
Free-Field:Marsh Edge & 1.000 \\
Free-Field:Habitat Complexity & 0.708 \\
Marsh Edge:Habitat Complexity & 0.719
\end{tabular}

\begin{tabular}{|c|c|}
\hline \multicolumn{2}{|l|}{ Polarization } \\
\hline Treatment Comparison & $\mathrm{P}$-value \\
\hline \multicolumn{2}{|l|}{ Habitat } \\
\hline Free-Field:Habitat Complexity & 0.954 \\
\hline Marsh Edge:Habitat Complexity & 0.743 \\
\hline Free-Field:Marsh Edge & 0.025 \\
\hline \multicolumn{2}{|l|}{ Pre-/Mid-Predator Attack } \\
\hline Free-Field & 0.928 \\
\hline Marsh Edge & 0.991 \\
\hline Habitat Complexity & 0.637 \\
\hline \multicolumn{2}{|l|}{ Pre-Attack } \\
\hline Free-Field:Marsh Edge & 0.855 \\
\hline Free-Field:Habitat Complexity & 0.765 \\
\hline Marsh Edge:Habitat Complexity & 1.000 \\
\hline \multicolumn{2}{|l|}{ Mid-Attack } \\
\hline Free-Field:Marsh Edge & 0.098 \\
\hline Free-Field:Habitat Complexity & 1.000 \\
\hline Marsh Edge:Habitat Complexity & 0.387 \\
\hline \multicolumn{2}{|l|}{ Correlation Strength } \\
\hline Treatment Comparison & $\mathrm{P}$-value \\
\hline \multicolumn{2}{|l|}{ Habitat } \\
\hline Free-Field:Habitat Complexity & 1.000 \\
\hline Marsh Edge:Habitat Complexity & 0.040 \\
\hline Free-Field:Marsh Edge & 0.002 \\
\hline \multicolumn{2}{|l|}{ Pre-/Mid-Predator Attack } \\
\hline Free-Field & 1.000 \\
\hline Marsh Edge & 0.998 \\
\hline Habitat Complexity & 1.000 \\
\hline \multicolumn{2}{|l|}{ Pre-Attack } \\
\hline Free-Field:Marsh Edge & 0.088 \\
\hline Free-Field:Habitat Complexity & 1.000 \\
\hline Marsh Edge:Habitat Complexity & 0.330 \\
\hline \multicolumn{2}{|l|}{ Mid-Attack } \\
\hline Free-Field:Marsh Edge & 0.279 \\
\hline Free-Field:Habitat Complexity & 1.000 \\
\hline Marsh Edge:Habitat Complexity & 0.678 \\
\hline
\end{tabular}

Table (3): Tukey post-hoc pairwise comparisons testing between each habitat, separating between pre-and mid-attack. 
Free-field, Pre-attack

\begin{tabular}{|c|c|c|c|c|}
\hline & $\mathrm{PCl}$ & $\mathrm{PC} 2$ & PC3 & PC4 \\
\hline Speed & -0.0687 & -0.6718 & -0.7364 & -0.0370 \\
\hline Area & 0.4935 & 0.2281 & -0.2730 & 0.0948 \\
\hline Angular Velocity & 0.4775 & 0.1566 & -0.2094 & 0.6590 \\
\hline Polarization & -0.4116 & 0.4333 & -0.3433 & -0.0642 \\
\hline Rotational Order & -0.3935 & 0.4552 & -0.3966 & 0.1728 \\
\hline Correlation Strength & 0.4465 & 0.2777 & -0.2536 & -0.7221 \\
\hline \multicolumn{5}{|l|}{ Free-field, Mid-attack } \\
\hline & $\mathrm{PCl}$ & $\mathrm{PC} 2$ & $\mathrm{PC} 3$ & $\mathrm{PC4}$ \\
\hline Speed & 0.0066 & -0.3701 & -0.9011 & -0.1768 \\
\hline Area & -0.5125 & -0.3060 & 0.1108 & 0.1799 \\
\hline Angular Velocity & -0.5074 & -0.2051 & -0.0367 & 0.6120 \\
\hline Polarization & 0.3775 & -0.5116 & 0.3187 & 0.0084 \\
\hline Rotational Order & 0.3726 & -0.5884 & 0.0970 & 0.2181 \\
\hline Correlation Strength & -0.4456 & -0.3455 & 0.2520 & -0.7170 \\
\hline \multicolumn{5}{|l|}{ Marsh Edge, Pre-attack } \\
\hline & $\mathrm{PCl}$ & $\mathrm{PC} 2$ & PC3 & PC4 \\
\hline Speed & 0.1861 & -0.0012 & 0.9201 & -0.2721 \\
\hline Area & -0.5461 & -0.1926 & -0.0064 & 0.1940 \\
\hline Angular Velocity & -0.4927 & -0.2808 & 0.2859 & 0.3666 \\
\hline Polarization & 0.2629 & -0.6636 & 0.1130 & 0.3828 \\
\hline Rotational Order & 0.2670 & -0.6383 & -0.2426 & -0.4217 \\
\hline Correlation Strength & -0.5329 & -0.1906 & -0.0018 & -0.6553 \\
\hline \multicolumn{5}{|c|}{ Marsh Edge, Mid-attack } \\
\hline & $\mathrm{PCl}$ & $\mathrm{PC} 2$ & $\mathrm{PC} 3$ & $\mathrm{PC4}$ \\
\hline Speed & -0.2606 & -0.3984 & -0.7988 & 0.2130 \\
\hline Area & 0.4945 & -0.2982 & 0.1617 & -0.0848 \\
\hline Angular Velocity & 0.4663 & -0.2969 & -0.2325 & -0.6545 \\
\hline Polarization & -0.3792 & -0.5447 & 0.1287 & -0.2467 \\
\hline Rotational Order & -0.3868 & -0.4163 & 0.4947 & -0.0673 \\
\hline Correlation Strength & 0.4203 & -0.4410 & 0.1435 & 0.6735 \\
\hline \multicolumn{5}{|c|}{ Habitat Complexity, Pre-attack } \\
\hline & $\mathrm{PCl}$ & $\mathrm{PC} 2$ & $\mathrm{PC} 3$ & PC4 \\
\hline Speed & 0.0413 & -0.4393 & 0.8787 & -0.0877 \\
\hline Area & -0.5702 & -0.2512 & -0.1224 & -0.2249 \\
\hline Angular Velocity & -0.5935 & 0.0867 & 0.0826 & -0.4708 \\
\hline Polarization & 0.1477 & -0.6241 & -0.1675 & 0.2196 \\
\hline Rotational Order & 0.2360 & -0.5158 & -0.4023 & -0.5130 \\
\hline Correlation Strength & -0.4933 & -0.2843 & -0.1269 & 0.6393 \\
\hline \multicolumn{5}{|c|}{ Habitat Complexity, Mid-attack } \\
\hline & $\mathrm{PCl}$ & $\mathrm{PC} 2$ & PC3 & $\mathrm{PC} 4$ \\
\hline Speed & -0.0971 & 0.1400 & -0.9844 & 0.0435 \\
\hline Area & 0.5148 & 0.2628 & -0.0245 & -0.0919 \\
\hline Angular Velocity & 0.5259 & 0.1981 & -0.0435 & -0.5435 \\
\hline Polarization & -0.3509 & 0.5882 & 0.1312 & 0.3011 \\
\hline Rotational Order & -0.3292 & 0.6116 & 0.0985 & -0.4756 \\
\hline Correlation Strength & 0.4664 & 0.3900 & 0.0395 & 0.6143 \\
\hline
\end{tabular}

Table (A1): PCA variable loadings for each habitat and attack condition. Schooling state parameter measurements are denoted in the first column, with principal components denoted along the first row. Scree plot analysis show that the first four principal components explain $>95 \%$ of the variability in the data. 
CHAPTER III

PREDATION-HABITAT COMPLEXITY COUPLING ALTERS COLLECTIVE STATE IN SCHOOLING FISH 


\begin{abstract}
Collective animal groups are known to exhibit behaviors that allow for effective responses to predators and environmental factors. Habitats with high levels of predation pressure or structural complexity have been shown to influence the collective tendencies of these animal aggregations. However, the effect of the coupling of habitat complexity and predation risk on collective tendency is still unknown. We examined the effect of increased perceived predation risk, as well as the coupling of habitat complexity and predation risk, in free ranging juvenile Gulf menhaden (Brevoortia patronus), a schooling forage fish. Using an acoustic imaging sonar, behavioral observations, prior to and during predator attacks, were collected in an open marsh canal as well as in the vicinity of a water control structure, known to act as an ecological hotspot, providing a simultaneous coupling of increased perceived risk and habitat structural complexity. We found that fish in schools responded to increased risk by becoming less aligned and cohesive with their school mates, and the coupling of predation risk and habitat complexity induced larger shoals with an increased ability to transfer social information. Our results demonstrate that collective tendency in schooling fish are affected not only by risk level or habitat complexity individually, but also by the coupling of the two conditions. Our study, thus, strengthens the idea that the complex environmental conditions animal groups live in may play a larger role in structuring collective behavior than each effect individually.
\end{abstract}

\title{
Background
}

Schooling fish are one of the many examples of organismal aggregations that perform complex collective behaviors (Parrish and Edelstein-Keshet, 1999; Partridge et 
al., 1980). These collective behaviors serve to provide the group a variety of benefits, including improved foraging, mate-selection, and enhanced predator evasion (Beauchamp, 2013; Parrish et al., 2002; Rieucau et al., 2015). Under threat of predation, schooling fish are known to exhibit a high degree of behavioral plasticity to the perceived risk of predation by performing a variety of collective anti-predator responses (Fernö et al., 1998; Parrish et al., 2002; Pitcher, 1983). For instance, previous studies have shown that changes at the collective level (e.g., greater levels of alignment between school members, increased swimming speed) are aimed at promoting an efficient transfer of threat-related information between neighboring fish that ultimately enhances the collective anti-predator reaction (Couzin et al., 2006; Parrish and Edelstein-Keshet, 1999; Partridge and Pitcher, 1980; Strandburg-Peshkin et al., 2013). In habitats with high levels of predation pressure, fish schools tend to form larger groups (Ioannou et al., 2017; Magurran et al., 1992; Seghers, 1974), exhibit more cohesion (Gerlotto et al., 2006; Huizinga et al., 2009; Song et al., 2011), and have a greater ability to transmit social information in response to a perturbation (Rieucau et al., 2016) than schools in low predation habitats. Previous work has also demonstrated the ability for fish schools to adjust schooling tendency relative to the perceived risk level (termed the "risk sensitive hypothesis") (Rieucau et al., 2014). Given the ubiquity of fish schooling in vastly different types of marine environments, and the behavioral plasticity in responses to perceived risk, the coupling of habitat structure and predation may affect and alter the collective response to predator attack.

In coastal estuarine systems, management efforts to mitigate flooding have led to the use of impoundments to regulate water flow in strategic areas along drainage canals. 
Anthropogenic impoundments have helped reduce losses of marsh edge habitats, mitigated the conversion of marsh habitat to open water, and stabilized the hydrology of these coastal systems (Montague et al., 1987; Rogers et al., 1994). However, water control structures (WCSs) have been shown to have detrimental ecological consequences, including migratory pattern interference in both juvenile and adult schooling fish (Rogers et al., 1992), habitat use modulation (Doehring et al., 2011; Wright et al., 2016), and decreased fish life-cycle success (Williams et al., 2012). The common solution to address these issues was the installation of slotted openings in WCSs that allowed for water flow and fish passage for nearly the complete water column, restoring access to, and connectivity with, the greater the estuarine habitat mosaic (Bunt et al., 2012; Kimball et al., 2010; Rulifson and Wall, 2006). Yet, the use of slotted WCSs in managed marshes did not promote unhindered fish passage; instead these slotted WCSs were found to act primarily as an aggregation area, rather than a passageway for free swimming fish (Kimball et al., 2015). These recent findings suggest that slotted WCSs are acting as an ecological hotspot, containing a three to six-fold increase in organismal abundance and density of both prey and predators around these WCSs, compared to an open salt marsh (Kimball et al., 2015, Kimball et al., 2018). The increases in abundance at the WCS, particularly for predatory fishes such as ladyfish (Elops saurus), red drum (Sciaenops ocellatus), and spotted seatrout (Cynoscion nebulosus) (Kimball et al., 2015, Kimball et al., 2017), implies that the likelihood of prey encountering predators is expected to be higher than in open water marshes.

Near these WCSs, the sensory information that fish are acting upon in the environment is not only influenced by the presence and complexity of the physical 
structure of the habitat, but also by an increase in perceived risk of predation. To a fish school, an increase in the abundance of predators would be perceived as a higher risk environment, yet an individual school would be unable to determine whether risk is reduced as a result of increased abundance of other fish schools. A fish school behaving in the proximity of a WCS would then need to contend with increased predation risk in a heterogeneous habitat while balancing other fitness enhancing activities such as foraging (Ioannou, 2017). Despite recent studies that have demonstrated that high-predation habitats influence social dynamics in fish schools (Ioannou et al., 2017), and that habitat complexity alters collective structure and schooling tendency (Rodriguez-Pinto 2020, in press), an open question remains whether the coupling of habitat structural complexity and higher perceived predation risk affects the schooling tendency in a manner that maximizes survival.

The objective of our study was to determine whether the behavioral schooling tendency of fish schools is modulated by the combination of high perceived predation risk and habitat complexity, prior to, and in response to predator attack. To achieve our objective, we compared the behavioral state of free-ranging schooling juvenile gulf menhaden (Brevoortia patronus), a planktivorous forage species that plays an important role as a source of prey for many piscivorous species, before and during predator attacks, between an open salt marsh canal and a WCS. The behavioral state of a fish school was quantified using six behavioral parameters commonly employed to quantify dynamic collective tendency in schooling fish: area occupied, group speed, angular velocity, orientational alignment (polarization), coherence of rotation (rotational order), and correlation strength (degree of influence on neighbors). We hypothesize that habitat 
complexity will elicit changes in the schooling state, but the schooling state will also be modulated in a way to enhance survival, as a result of increased perceived risk induced by the increased predation pressure. In risky conditions, schooling fish have been shown to exhibit greater speed (Gerlotto et al., 2006), increased density (Rieucau et al., 2015), swimming alignment, and higher correlation strength (Rieucau et al., 2016), than schools in non-risky conditions. Specifically, we predict that fish schools in the marsh canal and WCS will adjust their schooling tendencies according to the prevailing perception of risk (behavioral plasticity) and exhibit differences in area and polarization because of the expected differences in predation risk in the two habitats (WCSs being perceived as a risker habitat). Additionally, we predict that the coupling of both habitat complexity and increased perceived risk will induce changes in rotational order and correlation strength, to promote information transfer in the case of predator attack.

\section{Methods}

(a) Study Area \& Data Collection:

Our investigation builds upon prior studies that focused on in situ observations of nekton behavior and migration near WCSs (Kimball et al., 2010; Kimball et al., 2015; Kimball et al., 2017). In our study, behavioral observations of wild schooling fish were collected using an high-resolution imaging sonar (DIDSON, Sound Metrics, Inc.) at a WCS (Mangrove Bayou WCS; 29.8932N, 93.2309W), and a shallow ( 1.5 m) estuarine salt marsh canal $(29.8595 \mathrm{~N}, 93.2309 \mathrm{~W})$ in the Calcasieu Lake estuary, southwestern Louisiana, USA, between April and September 2010 (Figure 1). The salt marsh canal is $\sim 25 \mathrm{~m}$ wide, with a soft-sediment substrate, and is free of any structural complexity. 
Behavioral observations collected at the salt marsh canal acted as a free-field control to compare against observations at the WCS. The WCS is placed orthogonal to an off-lake canal channel, and totals $21 \mathrm{~m}$ in width. The WCS consisted of four bays (each $2.4 \mathrm{~m}$ wide) with a fixed-crest height of $1.4 \mathrm{~m}$. Acoustic imaging observations were collected at a single WCS bay (the far right bay, as viewed from the open water), that contained three open vertical slots $(0.15 \mathrm{~m} \times 1.2 \mathrm{~m})$ that allowed water flow and passage of swimming individuals (Figure 1) (Kimball et al., 2015).

The DIDSON sonar was attached to an adjustable mount approximately $0.5 \mathrm{~m}$ above the substrate. The DIDSON was operated at $1.8 \mathrm{MHz}$ at a tilt angle $-0.3^{\circ}$ to the surface, using a 96 -beam transducer array resulting in a $28^{\circ}$ by $14^{\circ}$ total field of view. Recordings were collected at a rate of 10 frames per second, with each frame mapping to an image with a range of $5 \mathrm{~m}$. At the WCS, the DIDSON was positioned $\sim 5 \mathrm{~m}$ from the slots, parallel to the flow of water, and all observations occurred within $2.5 \mathrm{~m}$ of the structure. In the free-field salt marsh canal, the DIDSON was centered in the water column and directed parallel to water flow as well (Figure 1).

Recordings were set at 4-hour intervals at both the WCS and adjacent free-field canal and were collected over consecutive days every month at the same point in the monthly and daily tidal cycle (daytime). Direct sampling of the region (Kimball et al., 2010; Kimball et al., 2015; Kimball et al., 2017) using cast nets (4.8-mm monofilament mesh; 2.4-m radius) during the recording period confirmed that prey schools were primarily comprised of B. patronus. Size measurements from DIDSON recordings are consistent with biological sampling data and confirmed the schools were B. patronus. 
Over 36 hours of recordings were analyzed for behavioral interactions between schooling fish and predators in both the free-field canal and WCS environments. Behavioral interactions were discovered and extracted in the free-field canal $(n=30)$ and at the WCS $(n=48)$. Each interaction extracted was separated into pre-and post-predator attack components. The pre/mid attack separation threshold for each behavioral interaction was chosen to coincide with the moment a predator penetrated the school. The attack duration was defined as the time interval from attack to when prey ceased responding to the predator (Pitcher, 1983), and the pre-attack duration was the equivalent interval prior to the separation threshold, enforcing equal time representation for preattack behavior and the subsequent anti-predator response. This resulted in a total of 156 behavioral interactions across the entire observation interval.

\section{(b) Data Processing:}

Each DIDSON acoustic sample is represented as a $512 \times 96$ frame. Initially, raw samples were wavelet denoised (MATLAB R2014b, Image Processing Toolbox) and transformed to distance-based images after correcting for acoustic beam spread. Image backgrounds were then estimated and subtracted from each image for processing. Particle Image Velocimetry (PIV), using a $32 \times 32$ pixel window was used to extract velocity estimates for the entire image (Westerweel, 1997). Subsequently, boundary detection was performed to extract the position of the fish school and isolate the velocity vectors that pertained to the behavior of the school. Particle image velocimetry velocity estimates were temporally filtered over a 5 -frame window, and spatially filtered using a $3 \times 3$ pixel median filter. Boundary detection was performed via a combination of image 
thresholding and pixel area-based filtering (MATLAB Image Processing Toolbox) (Otsu, 1979). Detected fish objects were segmented and labeled via distance based (0.3 m) cluster analysis (Pappas and Jayant, 1989). The school cluster boundary was then estimated using an alpha shape filter tuned to optimize shape area and perimeter. The final boundary was then tracked using a Kalman filter, and then approximated as a polygon to constrain the PIV velocity vectors to those pertaining to the fish school.

The school area was calculated using the school boundary detection algorithm, and is a local 2D projection approximation of the total area coverage occupied by the school (Handegard et al., 2012). The group speed was derived by center-of-mass measurements of the school and its change in position with respect to time. Angular velocity was computed via the curl of the PIV vectors that were located within the school. Polarization and rotational order were calculated from previously derived formulas from Attanasi et al. (2014). Polarization is the Euclidean norm of the sum of the velocity vectors normalized by magnitude (to isolate direction) divided by the total number of vectors. The rotational order measures the coherence of rotation in the school, quantified by summing the rotational components (projection of each rotational vector onto the axis orthogonal to the sonar plane) divided by its magnitude. Correlation strength (the degree to which one fish's behavior influences its neighbors) was calculated in the same manner as Rieucau et al. (2016).

\section{(c) Statistical Analysis:}

Each individual school observed was treated as an independent sample. To determine if the WCS or predation elicited differences in the schooling state, we 
conducted a two-way univariate ANOVA for each schooling state metric. Within the significant factors, Tukey post-hoc pairwise comparisons were conducted to determine significance (at 95\% significance level) of the mean schooling metrics, corrected for multiple comparisons. To visualize the strength and direction of the relationships between schooling state metrics, a generalized linear model with an identity link function was fitted for each predation state and habitat type. Model fits and associated model coefficients, and correlation estimates were derived. All analyses were completed using CRAN R statistical software (v3.6.2, "Dark and Stormy Night") (R. Development Core Team, 2005).

\section{Results}

(a) Habitat \& Predation Effects on Schooling State

When comparing between pre- and mid predator attack, we detected no significant differences in any of the schooling metrics (school area: $\mathrm{p}=0.13$, speed: $\mathrm{p}=$ 0.43 , angular velocity: $\mathrm{p}=0.34$, rotational order: $\mathrm{p}=0.29$, polarization: $\mathrm{p}=0.12$, correlation strength: $\mathrm{p}=0.94$ ), nor was an interaction effect observed between predation status and habitat context (area: $\mathrm{p}=0.65$, speed: $\mathrm{p}=0.56$, angular velocity: $\mathrm{p}=0.64$, rotational order: $\mathrm{p}=0.96$, polarization: $\mathrm{p}=0.86$, correlation strength: $\mathrm{p}=0.31)$. In contrast, we observed significant differences among all of the schooling metrics, with the exception of speed, between the free-field context and the WCS (school area: $\mathrm{p}=0.029$, $\mathrm{F}_{1,152}=4.8516$, angular velocity: $\mathrm{p}=0.003, \mathrm{~F}_{1,152}=8.992$, rotational order: $\mathrm{p}<0.001$, $\mathrm{F}_{1,152}=12.8729$, polarization: $\mathrm{p}=0.005, \mathrm{~F}_{1,152}=7.9531$, correlation strength: $\mathrm{p}<0.001$, $\left.\mathrm{F}_{1,152}=12.4871\right)($ Table A1). 
(b) Effect of Perceived Risk Increase and Habitat/Risk Coupling on Schooling State

When comparing within habitat context and between pre- and mid predator attack, we found significant differences in schooling metrics that reflect the change in perceived risk as well as the coupling of habitat complexity and increased risk. Prior to predator attack, we found a consistent significant decrease in school rotational order in the proximity of the WCS compared to the free-field (prior attack: $p=0.032$ ). We also found a significant decrease in polarization prior to attack $(\mathrm{p}=0.047)$ in the WCS compared to the free-field, which suggests that the increase in perceived risk has influenced the schooling state. During predator attack, the decrease in rotational order $(\mathrm{p}=0.017)$ and polarization remained consistent, although the decreasing pattern in polarization during a predator attack failed to reach the conventional level of statistical significance $(\mathrm{p}=$ 0.054). In addition, schools occupied a greater area $(\mathrm{p}=0.028)$, circularly swam approximately twice as fast $(\mathrm{p}=0.011)$, and exhibited a significant increase in correlation strength $(\mathrm{p}<0.002)$ near the WCS compared to the free-field canal (Figure 2, Table A1), which also suggests that in addition to the perceived risk effect, the coupling of habitat complexity and perceived risk further modulates the behavioral schooling state.

\section{(c) Risk level \& Habitat Effects on Correlation of Schooling State Metrics}

We observed significant, positive correlations between correlation strength and school area occupied in the free-field environment, both prior (Pearson correlation coefficient: $\mathrm{R}=0.78, \mathrm{p}<0.001)$ and during predator attack, $(\mathrm{R}=0.83, \mathrm{p}<0.001)$, as well as near the WCS, prior to $(\mathrm{R}=0.85, \mathrm{p}<0.001)$, and during $(\mathrm{R}=0.86, \mathrm{p}<0.001)$ predator attack. Similarly, we observed significant positive relationships between correlation 
strength and angular velocity in the free-field $(\mathrm{R}=0.79, \mathrm{p}<0.001$ pre-attack, $\mathrm{R}=0.8, \mathrm{p}$ $<0.001$ mid-attack $)$ and WCS $(\mathrm{R}=0.85, \mathrm{p}<0.001$ pre-attack, $\mathrm{R}=0.87, \mathrm{p}<0.001$ midattack). We also found a significant positive relationship between rotational order and polarization in the free field $(\mathrm{R}=0.83, \mathrm{p}<0.001$ pre-attack, $\mathrm{R}=0.67, \mathrm{p}<0.001$ midattack) and WCS $(\mathrm{R}=0.72, \mathrm{p}<0.001$ pre-attack, $\mathrm{R}=0.78, \mathrm{p}<0.001$ mid-attack) (Figure 3A). However, correlation strength and polarization/rotational order correlated differently between habitats and predator attack. Prior to predator attack, no significant correlation was found between correlation strength and polarization/rotational order in the free field. In the proximity of the WCS, however, we detected a significant, negative correlation between correlation strength and polarization $(\mathrm{R}=-0.55, \mathrm{p}<0.001)$ and correlation strength and rotational order $(\mathrm{R}=-0.43, \mathrm{p}=0.002)$, suggesting a shift in schooling state relative to the increase in perceived risk. During predator attack, we found that correlation strength and polarization were negatively correlated $(\mathrm{R}=-0.73, \mathrm{p}<$ $0.001)$ as well as correlation strength and rotational order $(\mathrm{R}=-0.62, \mathrm{p}<0.001)$ in the free-field, and at the WCS (polarization: $\mathrm{R}=-0.66, \mathrm{p}<0.001$, rotational order: $\mathrm{R}=-$ $0.63, p<0.001$ ), demonstrating the effect of habitat structure on the schooling state in both low risk and high risk conditions (Figure 3B). Finally, we observed an increase in correlation between polarization and school area during predator attack in the free field $(\mathrm{R}=-0.37, \mathrm{p}=0.045$ pre-attack, $\mathrm{R}=-0.67, \mathrm{p}<0.001$ mid-attack $)$, which was not observed in the proximity of the WCS $(\mathrm{R}=-0.49, \mathrm{p}<0.001$ pre-attack, $\mathrm{R}=-0.54, \mathrm{p}<$ 0.001 mid-attack) (Figure 3B). 


\section{Discussion}

Our results demonstrate that collective tendencies of fish schools are driven not only by perceived threat level, or habitat structural complexity, but by the coupling of the two conditions. The differences found in each schooling state metric (except speed) between the free-field canal and the water control structure suggest that the combination of spatial complexity from the physical structure with the increase in perceived threat are driving changes in collective behavior. At the higher risk environment created by the WCS, the decrease in rotational order and polarization of schools compared to the lower risk free-field environment implies that schools exhibit less alignment and coherence in rotation even in the face of increased threat. This result contrasts previous work that showed increased alignment and cohesion in riskier conditions (Rieucau et al., 2016), and tighter schooling with an increase in both rotational order and polarization in habitats with a structural boundary (Rodriguez-Pinto 2020, in press).

The differences in schooling state between the environments prior to predator attack demonstrate the magnitude of the threat sensitive response on the schooling tendency. However, the additional changes in schooling state during predator attack suggest that the coupling of both increased risk and habitat complexity has a major role in structuring the collective tendency. In our case, the risk effect-linked adoption of lower alignment and rotational cohesion behavioral tendencies are maintained, while also favoring a looser, more shoal-like composition, with an increase in the ability to transmit social information due to the coupling of increased risk and habitat complexity. The overall increase in correlation strength supports the idea of increased vigilance in a riskier, complex environment, where each individual will maximize its capability to 
respond to indirect information transferred via conspecifics (Couzin et al., 2006; Strandburg-Peshkin et al., 2013; Ward et al., 2011), driven by the "threat-sensitive hypothesis" (Rieucau et al., 2014). The coupling of the two effects then becomes the dominant external factor that modulates the schooling state, and drives the collective tendency in a different manner than predation risk or habitat complexity alone. From a sensory perspective, perceived motion on the retina is much higher for a dynamic object than a static one, and an increase in predation risk may shift the sensory field to respond more strongly to fast moving objects, relative to the increase in threat (Brenner, 1991; Land, 1988; Münch et al., 2009). However, the static presence of the structure may influence the sensory field as well (Land and Nilsson, 2012; Ölveczky et al., 2003); further investigation is required to determine the effect of these coupled effects on the visual component of schooling.

Although we found differences in schooling state between the free-field and WCS, many correlations between schooling state metrics remain robust to the presence of habitat complexity and higher risk, and do not change in either the direction or strength of their correlative relationships. The main significant positive correlations between correlation strength with area and angular velocity, as well as the significant positive correlation between rotational order and polarization, was well conserved between predator attack and habitat context, illustrating the behavioral plasticity of the schooling response. However, we also detected changes in correlations between schooling metrics as a result of predator attack, and further changes with the coupling of both habitat complexity and increased perceived risk. In the free-field environment, schools elicited negative correlations between polarization and correlation strength, as well as rotational 
order and correlation strength, only during predator attack. However, the negative correlations occur at the WCS, regardless of predator attack, demonstrating that predation or habitat context may drive collective tendency in different manners. The coupling of habitat complexity and a higher level of perceived risk at the WCS also created a strong correlation between polarization and school area, that is only weakly present in the freefield habitat prior to predator attack, suggesting that the schooling tendency may be modulated in a more complex manner than the sum of the individual effects. In this case, the coupling of the two effects drives collective tendency in a manner only observed in open environments during predator attack. Although we can clearly see the effect of risk level and structural complexity coupling on schooling, analyzing schooling behavior in the presence of the WCS poses an inherent inability to decouple the individual effects of perceived risk and habitat structure. Further studies are needed to truly decouple and ascertain the specific contribution of the role of perceived risk and habitat structure on collective tendency of free-ranging schooling fish.

Our investigation examined the coupled effect of structural habitat complexity and increased perceived predation risk on behavioral responses of fish schools before and during predator attack. We found that the drivers of collective tendencies are more complex in natural systems; collective tendencies are not only driven by predation and predation risk, but also by habitat characteristics and their coupling. Our study, conducted in situ, observed behavioral responses in truly natural conditions, which incorporates all of the potential modulators of collective state, including predation and habitat complexity, and shows that environmental characteristics play a large role in structuring collective behavior. This study further demonstrates the potential for external abiotic and 
biotic factors to affect structure at the collective level and assess the impact of habitat on trophic interactions, while providing a better understanding beyond the fine scale of collective animal processes.

Animal Ethics \& Fieldwork Statement:

This research was conducted in accordance with the guidelines set forth in the Louisiana State University IACUC Animal Care and Use Protocols \#10-115 and \#11-090.

Acknowledgements

We thank S. Labua for technical and advisory help during the project conception phase and L. Broussard for assistance in the fieldwork.

References

Attanasi, A., Cavagna, A., Del Castello, L., Giardina, I., Melillo, S., Parisi, L., Pohl, O., Rossaro, B., Shen, E., Silvestri, E., et al. (2014). Collective Behaviour without Collective Order in Wild Swarms of Midges. PLoS Computational Biology 10, e1003697.

Beauchamp, G. (2013). Social predation: how group living benefits predators and prey. Elsevier.

Brenner, E. (1991). Judging object motion during smooth pursuit eye movements: The role of optic flow. Vision Research 31, 1893-1902.

Bunt, C. M., Castro-Santos, T. and Haro, A. (2012). Performance Of Fish Passage Structures At Upstream Barriers To Migration: Performance Of Fish Passage Structures. River Research and Applications 28, 457-478.

Couzin, I. D., James, R., Mawdsley, D., Croft, D. P. and Krause, J. (2006). Social organization and information transfer in schooling fishes. Fish cognition and behavior 166-185. 
Doehring, K., Young, R. G. and McIntosh, A. R. (2011). Factors affecting juvenile galaxiid fish passage at culverts. Marine and Freshwater Research 62, 38.

Fernö, A., Pitcher, T. J., Melle, W., Nøttestad, L., Mackinson, S., Hollingworth, C. and Misund, O. A. (1998). The challenge of the herring in the Norwegian sea: Making optimal collective spatial decisions. Sarsia 83, 149-167.

Gerlotto, F., Bertrand, S., Bez, N. and Gutierrez, M. (2006). Waves of agitation inside anchovy schools observed with multibeam sonar: a way to transmit information in response to predation. ICES Journal of Marine Science 63, 1405-1417.

Handegard, N. O., Boswell, K. M., Ioannou, C. C., Leblanc, S. P., Tjøstheim, D. B. and Couzin, I. D. (2012). The Dynamics of Coordinated Group Hunting and Collective Information Transfer among Schooling Prey. Current Biology 22, 1213-1217.

Huizinga, M., Ghalambor, C. K. and Reznick, D. N. (2009). The genetic and environmental basis of adaptive differences in shoaling behaviour among populations of Trinidadian guppies, Poecilia reticulata. Journal of Evolutionary Biology 22, 1860-1866.

Ioannou, C. C. (2017). Grouping and Predation. In Encyclopedia of Evolutionary Psychological Science (ed. Shackelford, T. K.) and Weekes-Shackelford, V. A.), pp. 1-6. Cham: Springer International Publishing.

Ioannou, C. C., Ramnarine, I. W. and Torney, C. J. (2017). High-predation habitats affect the social dynamics of collective exploration in a shoaling fish. Science Advances 3, e1602682.

Kimball, M. E., Rozas, L. P., Boswell, K. M. and Cowan, J. H. (2010). Evaluating the effect of slot size and environmental variables on the passage of estuarine nekton through a water control structure. Journal of Experimental Marine Biology and Ecology 395, 181-190.

Kimball, M. E., Rozas, L. P., Boswell, K. M. and Cowan Jr, J. H. (2015). Effects of slotted water control structures on nekton movement within salt marshes. Marine and Coastal Fisheries 7, 177-189.

Kimball, M. E., Boswell, K. M. and Rozas, L. P. (2017). Estuarine fish behavior around slotted water control structures in a managed salt marsh. Wetlands Ecology and Management 25, 299-312.

Kimball, M. E., Boswell, K. M., Rozas, L. P., Berwaldt, E. K. and Richards, A. R. (2018). Swimming abilities of juvenile estuarine fishes: implications for passage at water control structures. Wetlands Ecology and Management 26, 383-390.

Land, M. F. (1988). The optics of animal eyes. Contemporary Physics 29, 435-455. 
Land, M. F. and Nilsson, D.-E. (2012). Animal eyes. Oxford University Press.

Magurran, A., Seghers, B. H., Carvalho, G. R. and Shaw, P. W. (1992). Behavioural consequences of an artificial introduction of guppies (Poecilia reticulata) in $\mathrm{N}$. Trinidad: evidence for the evolution of anti-predator behaviour in the wild. Proc. R. Soc. B 248, 117-122.

Montague, C., Zale, A. and Percival, H. (1987). Ecological effects of coastal marsh impoundments: A review. Environmental Management 11, 743-756.

Münch, T. A., da Silveira, R. A., Siegert, S., Viney, T. J., Awatramani, G. B. and Roska, B. (2009). Approach sensitivity in the retina processed by a multifunctional neural circuit. Nat Neurosci 12, 1308-1316.

Ölveczky, B. P., Baccus, S. A. and Meister, M. (2003). Segregation of object and background motion in the retina. Nature 423, 401-408.

Otsu, N. (1979). A threshold selection method from gray-level histograms. IEEE transactions on systems, man, and cybernetics 9, 62-66.

Pappas, T. N. and Jayant, N. S. (1989). An adaptive clustering algorithm for image segmentation. In International Conference on Acoustics, Speech, and Signal Processing, pp. 1667-1670. IEEE.

Parrish, J. K. and Edelstein-Keshet, L. (1999). Complexity, pattern, and evolutionary trade-offs in animal aggregation. Science 284, 99-101.

Parrish, J. K., Viscido, S. V. and Grunbaum, D. (2002). Self-organized fish schools: an examination of emergent properties. The biological bulletin 202, 296-305.

Partridge, B. L. and Pitcher, T. J. (1980). The sensory basis of fish schools: relative roles of lateral line and vision. Journal of Comparative Physiology 135, 315-325.

Partridge, B. L., Pitcher, T., Cullen, J. M. and Wilson, J. (1980). The three-dimensional structure of fish schools. Behavioral Ecology and Sociobiology 6, 277-288.

Pitcher, T. J. (1983). Heuristic definitions of fish shoaling behaviour. Animal Behaviour.

R. Development Core Team (2005). R: A language and environment for statistical computing, reference index version 2.2. 1. R Foundation for Statistical Computing, Vienna, Austria.

Rieucau, G., Boswell, K. M., De Robertis, A., Macaulay, G. J. and Handegard, N. O. (2014). Experimental Evidence of Threat-Sensitive Collective Avoidance Responses in a Large Wild-Caught Herring School. PLoS ONE 9, e86726. 
Rieucau, G., Fernö, A., Ioannou, C. C. and Handegard, N. O. (2015). Towards of a firmer explanation of large shoal formation, maintenance and collective reactions in marine fish. Reviews in Fish Biology and Fisheries 25, 21-37.

Rieucau, G., Holmin, A. J., Castillo, J. C., Couzin, I. D. and Handegard, N. O. (2016). School level structural and dynamic adjustments to risk promote information transfer and collective evasion in herring. Animal Behaviour 117, 69-78.

Rogers, D. R., Rogers, B. D. and Herke, W. H. (1992). Effects of a marsh management plan on fishery communities in Coastal Louisiana. Wetlands 12, 53-62.

Rogers, D. R., Rogers, B. D. and Herke, W. H. (1994). Structural marsh management effects on coastal fishes and crustaceans. Environmental Management 18, 351369.

Rulifson, R. A. and Wall, B. L. (2006). Fish and Blue Crab Passage through Water Control Structures of a Coastal Bay Lake. North American Journal of Fisheries Management 26, 317-326.

Seghers, B. H. (1974). Schooling Behavior in the Guppy (Poecilia reticulata): An Evolutionary Response to Predation. Evolution 28,.

Song, Z., Boenke, M. C. and Rodd, F. H. (2011). Interpopulation Differences in Shoaling Behaviour in Guppies (Poecilia reticulata): Roles of Social Environment and Population Origin: Shoaling Behaviour in Guppies. Ethology 117, 1009-1018.

Strandburg-Peshkin, A., Twomey, C. R., Bode, N. W., Kao, A. B., Katz, Y., Ioannou, C. C., Rosenthal, S. B., Torney, C. J., Wu, H. S., Levin, S. A., et al. (2013). Visual sensory networks and effective information transfer in animal groups. Current Biology 23, R709-R711.

Ward, A. J. W., Herbert-Read, J. E., Sumpter, D. J. T. and Krause, J. (2011). Fast and accurate decisions through collective vigilance in fish shoals. Proceedings of the National Academy of Sciences 108, E27-E27.

Westerweel, J. (1997). Fundamentals of digital particle image velocimetry. Meas. Sci. Technol. 8, 1379-1392.

Williams, J. G., Armstrong, G., Katopodis, C., Larinier, M. and Travade, F. (2012). Thinking Like A Fish: A Key Ingredient For Development Of Effective Fish Passage Facilities At River Obstructions: Fish Behaviour Related Fish Passage At Dams. River Research and Applications 28, 407-417.

Wright, G. V., Wright, R. M., Bendall, B. and Kemp, P. S. (2016). Impact of tide gates on the upstream movement of adult brown trout, Salmo trutta. Ecological Engineering 91, 495-505. 
Figures

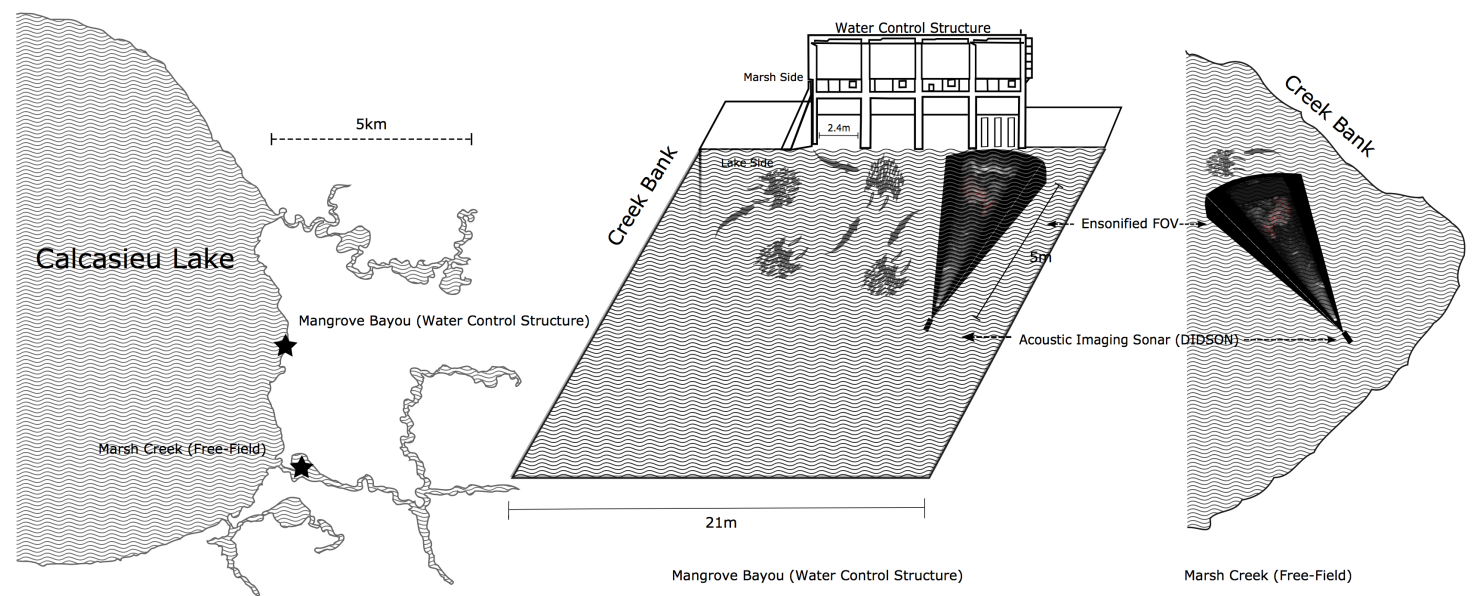

Figure (1): Schematic representation of the study site along with the habitat conditions where behaviors were observed. The two test sites are denoted in the larger scale map by a star, with each habitat illustrated in the adjacent panel. The marsh creek acted as a freefield, lower predation risk control environment. The water control structure acts as an ecological hotspot, with increased predation risk in addition to the habitat structural complexity. 

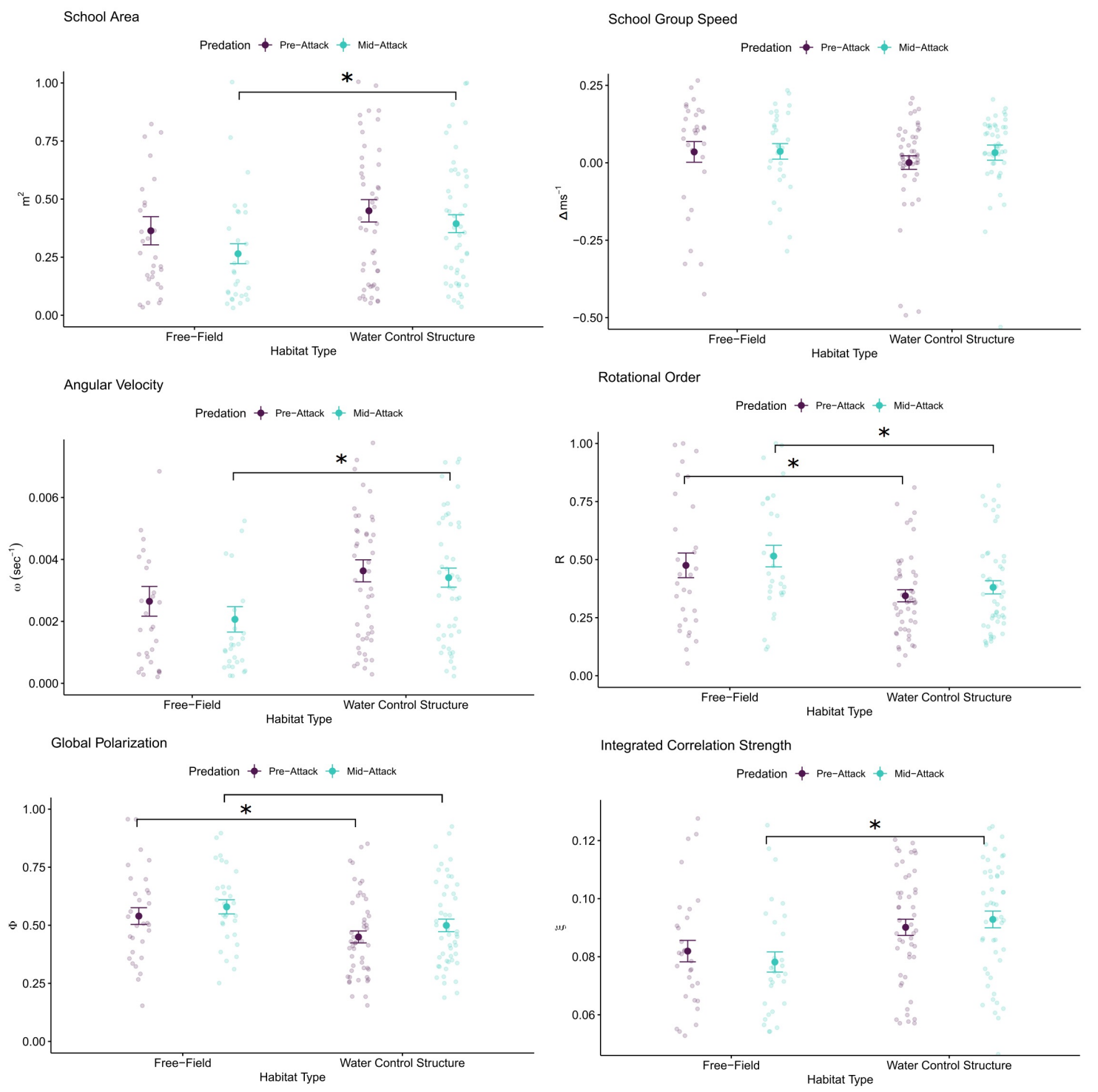

Figure (2): Mean comparisons of six behavioral metrics that describe schooling tendency between habitat context and predator attack. Habitat context comparisons, separated on the bottom axis are separated between pre and mid predator attack (separated by color), presenting comparisons between and within habitat context. Significant differences at 95\% confidence level are denoted with an asterisk. 

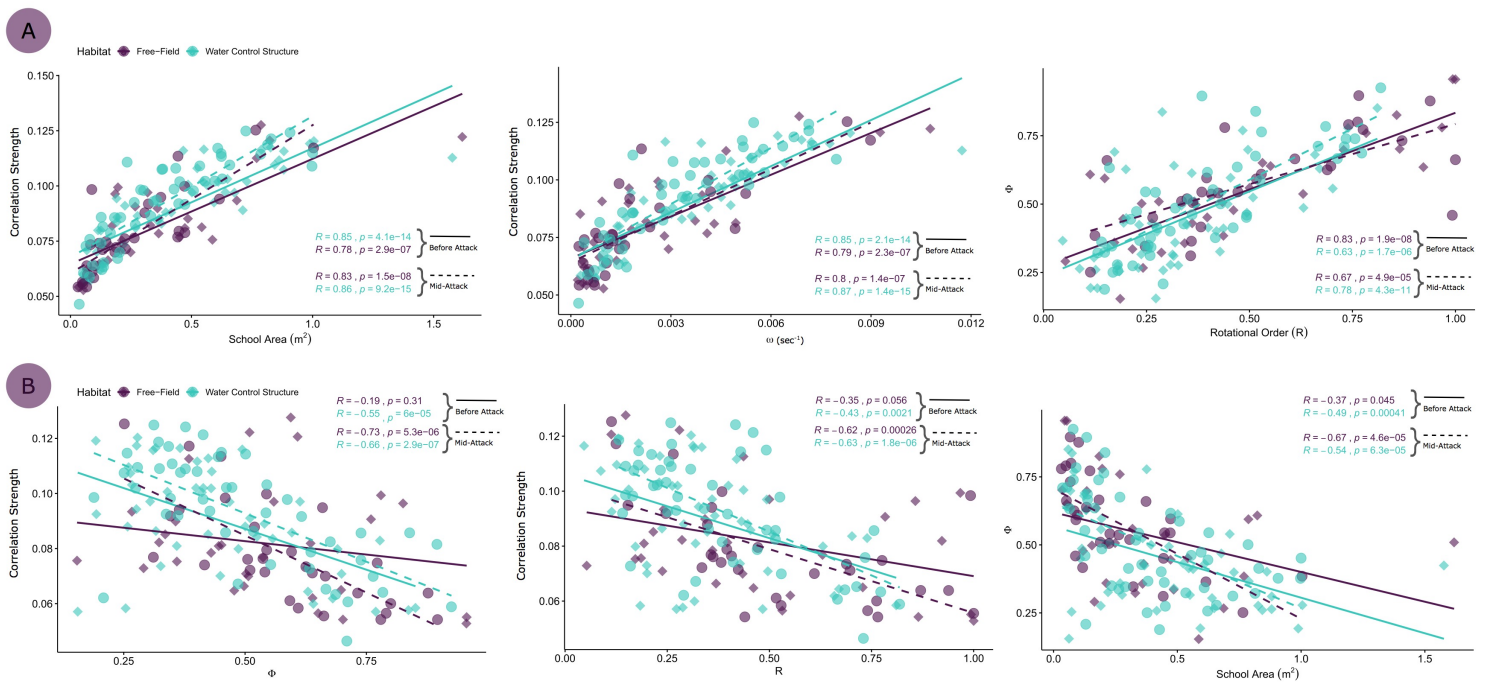

Figure (3A, 3B): Generalized Linear Model fits with identity link functions for relevant pairs of schooling metrics. Fit lines and associated correlation coefficients were calculated from the GLM model for each combination of habitat context and predator attack status. Habitat context data are separated by color, and predation status data are denoted by data point shape. Model fits for habitat context are also separated by color, and model fits for predator attack are differentiated by solid and dashed lines. Significant fits and associated correlation values are reported in the upper right-hand corner of each plot. 
School Area

\begin{tabular}{lrrrr}
\hline & df & SS & F & p-value \\
\hline Predation & 1 & 0.2032 & 2.3040 & 0.1311 \\
Habitat & 1 & 0.4280 & 4.8516 & 0.0291 \\
Predation:Habitat & 1 & 0.0174 & 0.1967 & 0.6580 \\
Residuals & 152 & 13.4081 & &
\end{tabular}

\begin{tabular}{lrrrr}
\multicolumn{3}{c}{ Treatment Comparison } & & P-value \\
\cline { 1 - 3 } Pre-/Mid-Predator Attack & & \\
Free-Field & & 0.189 \\
Water Control Structure & & 0.371 \\
Free-Field/Water Control Structure & \\
Pre-Attack & & 0.272 \\
Mid-Attack & & 0.028 \\
\multicolumn{4}{c}{ Angular Velocity } & \\
\hline & df & SS & F & p-value \\
\hline Predation & 1 & 0.000005 & 0.902819 & 0.343535 \\
Habitat & 1 & 0.000050 & 8.991612 & 0.003170 \\
Predation:Habitat & 1 & 0.000001 & 0.217613 & 0.641532 \\
Residuals & 152 & 0.000847 & &
\end{tabular}

\begin{tabular}{lrrrr}
\multicolumn{5}{c}{ Group Speed } \\
\hline & df & SS & F & p-value \\
\hline Predation & 1 & 0.0163 & 0.6250 & 0.4304 \\
Habitat & 1 & 0.0137 & 0.5276 & 0.4687 \\
Predation:Habitat & 1 & 0.0086 & 0.3291 & 0.5670 \\
Residuals & 152 & 3.9539 & &
\end{tabular}

\begin{tabular}{lc}
\multicolumn{1}{c}{ Treatment Comparison } & P-value \\
\cline { 1 - 1 } Pre-/Mid-Predator Attack & \\
Free-Field & 0.968 \\
Water Control Structure & 0.331 \\
Free-Field/Water Control Structure & \\
Pre-Attack & 0.392 \\
Mid-Attack & 0.908 \\
\multicolumn{2}{c}{ Rotational Order } \\
\hline
\end{tabular}

\begin{tabular}{lrrrr}
\hline & df & SS & F & p-value \\
\hline Predation & 1 & 0.0558 & 1.1031 & 0.2953 \\
Habitat & 1 & 0.6508 & 12.8729 & 0.0004 \\
Predation:Habitat & 1 & 0.0001 & 0.0022 & 0.9628 \\
Residuals & 152 & 7.6850 & &
\end{tabular}

\begin{tabular}{ll}
\multicolumn{1}{c}{ Treatment Comparison } & P-value \\
\cline { 1 - 1 } Pre-/Mid-Predator Attack & \\
Free-Field & 0.573 \\
Water Control Structure & 0.347 \\
Free-Field/Water Control Structure & \\
Pre-Attack & 0.032 \\
Mid-Attack & 0.017
\end{tabular}

\begin{tabular}{lrrrr}
\multicolumn{5}{c}{ Correlation Strength } \\
& df & SS & F & p-value \\
\hline Predation & 1 & 0.0000 & 0.0053 & 0.9420 \\
Habitat & 1 & 0.0048 & 12.4871 & 0.0005 \\
Predation:Habitat & 1 & 0.0004 & 0.9990 & 0.3191 \\
Residuals & 152 & 0.0587 & &
\end{tabular}

\begin{tabular}{ll}
\multicolumn{1}{c}{ Treatment Comparison } & P-value \\
\cline { 1 - 1 } Pre-/Mid-Predator Attack & \\
Free-Field & 0.462 \\
Water Control Structure & 0.501 \\
Free-Field/Water Control Structure & \\
Pre-Attack & 0.082 \\
Mid-Attack & 0.002
\end{tabular}

Table (1): Two-way ANOVA results with subsequent post-hoc pairwise comparisons for each of the six schooling metrics describing schooling tendency. ANOVA tables testing for effect of habitat context, predation, and their interaction are reported above, while Tukey post-hoc pairwise comparisons within habitat context and predation conditions are reported below. 


\section{CHAPTER IV}

ENVIRONMENTAL IMPACTS ON VISUAL PERCEPTION MODULATES BEHAVIORAL RESPONSES OF SCHOOLING FISH TO LOOMING PREDATORS 


\begin{abstract}
Aggregation adaptations in socially living fishes have primarily evolved to improve safety from predators. The individual interaction mechanisms that govern collective behavior in fish schools are determined by the sensory systems that translate environmental information into behavior. In dynamic environments, shifts in environmental conditions impede the ability for effective visual sensory perception in fish schools, and may induce changes in the collective response. Here, we consider whether environmental conditions that affect visual contrast modulate the collective response of schooling fish to looming predators. By using a virtual environment to simulate four different contrast levels, we tested whether the collective state of fish schools (and thus the interaction mechanisms) was modified in response to a looming optical stimulus. Our results indicate that fish swam slower and were less polarized in lower contrast conditions. In addition, schooling metrics known to be regulated by nonvisual sensory systems became more correlated with decreasing contrast. Over the course of the escape response, schools remained more tightly formed and retained the capability of transferring social information. Results suggest that when visual perception is compromised, the interaction rules governing collective behavior are modified to prioritize ancillary sensory information crucial to maximizing chance of escape. Finally, these results imply that multiple sensory systems can integrate to control collective behavior in environments with unreliable visual information.
\end{abstract}




\section{Background}

Schooling in fish confers safety benefits that maximize probability of survival (Pitcher and Parrish, 1993). In social fish, this is likely motivated by the threat of predation (Ioannou, 2017), where schooling can improve survival by diluting risk (Hoare et al., 2004; Turner and Pitcher, 1986), decrease encounter rate with predators (Ioannou et al., 2011), and enhance group vigilance (Ward et al., 2011). When directly responding to predators, schools can exhibit different behaviors to evade predators (Magurran and Pitcher, 1987), including reducing inter-fish distance (Hoare et al., 2004), and initiating escape waves (Herbert-Read et al., 2015). However, the interactions between wild schooling fish and their aquatic predators occur in a wide range of environmental conditions, from turbulent coral reef to turbid estuarine waters, which may alter the ability for an effective anti-predator response (Higham et al., 2015).

Water turbidity (Abrahams and Kattenfeld, 1997) and light availability (Land, 1988) are well-known to affect schooling tendency (Chamberlain and Ioannou, 2019; Ryer and Olla, 1998). In estuarine and riverine waters, turbidity impacts prey fish visual detection (Utne-Palm, 2002), plays a critical role in structuring predator-prey interactions (Abrahams and Kattenfeld, 1997; De Robertis et al., 2003), and decreases the ability for schools to perform successful antipredator responses (Figueiredo et al., 2016; Kimbell and Morrell, 2015). Previous studies have linked how sensory information and perception translates into escape responses in animals (Fotowat and Gabbiani, 2011), which supports the hypothesis that environmental factors (through changes in sensory perception) can directly influence antipredator responses. It has been demonstrated that the visual sensory system plays a key role in initiating and directing escape behaviors in 
both individual (Hein et al., 2018; Temizer et al., 2015) and schooling prey fish (Rosenthal et al., 2015). From a physiological perspective, the fish eye has largely evolved to maximize light-gathering power (Land, 2005); therefore environmental changes that affect light availability (e.g., turbidity) may actively compromise visual perception and influence antipredator schooling responses. In light restricted or turbid environments, vigilant schooling fish under attack must respond to a decrease in perceived visual contrast, where the ability to distinguish a predator from the background environment becomes increasingly more difficult as contrast decreases (Land and Nilsson, 2012). However, it is currently unknown whether decreasing visual contrast impacts collective mechanisms that mediate group responses to predator attacks.

To a freely behaving prey fish, visual detection of a fast approaching predator is perceived by the visual system as a looming optical stimulus (Bhattacharyya et al., 2017; Cade et al., 2020; Temizer et al., 2015). These looming stimuli have been shown to reliably initiate escape maneuvers in fish both in laboratory settings (Cade et al., 2020) and in the wild (Hein et al., 2018). Escape behaviors to looming stimuli are also well conserved across many animal species (Peek and Card, 2016). A looming stimulus consists of an expanding image that simultaneously triggers both spatial and temporal motion detecting neurons (Bhattacharyya et al., 2017; Temizer et al., 2015) in the visual system. The optic flow on the retina by an expanding object can be affected by both the speed and rate of stimulus expansion, as well as the luminosity of the object and the contrast between object and background (Bhattacharyya et al., 2017). Temizer et al., (2015) demonstrated that luminance affects both escape and other responses to looming 
stimuli. A decrease in contrast of a looming stimulus may then affect the ability and magnitude of escape responses in fish schools.

Many studies have investigated the mechanisms of interaction in schooling fish (Conradt, 2012; Gautrais et al., 2012), and in behavioral studies, used motion based metrics to quantify behavioral responses (Delcourt and Poncin, 2012). Common motion metrics to describe dynamic collective behavior include nearest neighbor distance (Parrish et al., 2002), average swimming speed (Berdahl et al., 2013; Kent et al., 2019; Zienkiewicz et al., 2018), orientational polarization (Cavagna et al., 2008; Viscido et al., 2004), and correlation length as a measure of information transfer rate (Cavagna et al., 2010; Handegard et al., 2012). Previous efforts have proposed simplified interaction models to simulate how an individual in the aggregation behaviorally responds to its immediate neighbors (Couzin, 2009; Couzin et al., 2002; Katz et al., 2011; StrandburgPeshkin et al., 2013), which include positioning and speed within the school, as well as alignment between individuals. However, the underlying interaction mechanisms that control the behavioral state of a freely-behaving fish school are much more complex, and are influenced by variation in their local environment (Berdahl et al., 2013; Tunstrøm et al., 2013). Efforts at modeling individual interaction rules determined that the visual field is a large contributor to accurately replicating the behavioral patterns that control schooling state (Bastien and Romanczuk, 2020; Collignon et al., 2016; StrandburgPeshkin et al., 2013). In freely behaving schools, fish utilize a combination of vision and mechanosensation to control schooling. Vision largely controls velocity regulation and orientation (Partridge and Pitcher, 1980), while the lateral line system controls interindividual spacing (Faucher et al., 2010) and the ability to detect movement of 
neighboring individuals (Mogdans and Bleckmann, 2012; Montgomery et al., 1995). In some species of prey fish, the lateral line mechanosensory system plays a key role in predator detection (Stewart et al., 2014), and may become more important when schools are in an environment that limits visual contrast (Montgomery et al., 1995; Partridge and Pitcher, 1980). Therefore, determining whether contrast changes modulate interaction mechanisms of fish schools in response to looming predators will further inform the contribution of vision to the mechanisms of collective behavior.

The objective of this study is to determine whether abiotic factors affecting contrast, the extent to which the luminance of the stimulus differs from the environment (Land and Nilsson, 2012), modulate the collective interaction mechanisms of schooling fish in response to looming predators. We hypothesize that schooling behavior (and their interaction mechanisms) may be affected by contrast decreases in three ways: 1) compromised vision results in changes in interaction mechanisms that elicit weaker behavioral responses, 2) low contrast conditions induce changes in interaction mechanisms to favor auxiliary sensory systems (such as the lateral line) that compensate for vision loss, or 3) contrast effects on vision are negligible and have no influence on schooling behavior. To determine how stimuli-environment luminance contrast influences schooling behavior, we quantified the collective state of fish schools (spp. Pimephales promelas), using five physical metrics, in response to looming optical stimuli at four decreasing contrast levels. 


\section{Methods}

\section{(a) Experimental Setup \& Data Collection:}

The experimental tank was a perspex cube, with $228 \mathrm{~mm}$ sides covered with backprojection screen material, filled to $100 \mathrm{~mm}$ depth. Two first-surface mirrors, angled at 45 deg to each side, allow a single projector (Lightspeed Designs DepthQ 360) to illuminate two sides of the tank, an additional two mirrors, orthogonal to each other, were angled at 45 degrees to illuminate the rear side (Fig. 1). We used custom OpenGL software to animate the cube with three dimensional, perspective-corrected scenes at 360 frames s-1. Within the cube, the virtual environment appears continuous, even as the looming stimulus transitions from one surface to another (Fig. 1). The four rendered scenes that reflected off the mirrors were mathematically reversed to account for the reflection (Cabrera and Theobald, 2013). The front face displayed $229 \times 229$ pixels $(2.5$ pixels deg-1) and the side faces displayed $200 \times 200$ (2.2 pixels deg-1). The small difference is due to the shorter path between the projector and the front surface, which displays an image of the same size but at a higher pixel density. During the course of the experiments, each side was illuminated uniformly with white light and the looming stimulus was represented as a black disk for maximum contrast. The disk expanded at a constant rate, passed through the tank holding the schools, and contracted on the opposite surface, simulating a direct visual predator attack. Looming stimulus direction was randomized among the four cardinal directions, to prevent habituation. Contrast was manipulated by adding "visual turbidity"- 1mm radius black dots at randomly determined positions on each surface, with each contrast level increasing in dot density (representing contrast increases of $1 / 4$ ) according to an exponential growth scale. In our experiment, the 
high contrast condition contained a single $1 \mathrm{~mm}$ dot, and the low contrast condition containing 10,000 dots across all surfaces (Figure 1). From within the cube, the higher the dot density, the lower the contrast between the looming stimulus and the environment. An overhead camera captured video footage of behavioral responses recording at 180 frames per second, with an infrared illumination source below the tank for improved image visualization and ease of data processing in low contrast conditions.

Prior to each experiment, we randomly selected schools of 8-12 fathead minnow from a larger population of acclimated fish and placed in the experimental tank for 10 minutes. Each school was exposed to four simulated predator attack trials, from random directions, at a given contrast level. The time span of each attack was approximately $\sim 6 \mathrm{~s}$, and the behavioral response interval was determined from the first frame showing stimulus expansion to the last frame showing stimulus contraction. Once the trials were completed each school was returned to the tank and given a minimum of 30 minutes to reacclimate. Subsequent experiments used random samples from the larger population to ensure that no two schools recorded were identical, even though single fish may have been tested more than once. In total, we observed 161 different shoals across the four contrast levels, with observations recorded from a total of 1457 individual fish.

\section{(b) Data Processing:}

After data collection, each experiment was partitioned into its respective behavioral response trials. Due to the high framerate of the camera, individual frames for analysis were subsampled at $10 \mathrm{hz}$ across the response interval. Tail and head points were manually identified for each fish in the school, for every subsampled image frame, 
providing an angle and position for each fish in the school. Using the position and angle values, we quantified five parameters of the collective response: 1) nearest neighbor distance, 2) individual velocity, 3) angle to nearest neighbor, 4) school polarization, and 5) correlation length. To estimate nearest neighbor distance, we performed a k-nearest neighbors (k-NN) search, based on Euclidean distance between positions, for each individual fish in each frame. Velocity was calculated by initially tracking individual fish positions using $\mathrm{k}-\mathrm{NN}$ search between successive frames for each fish across the entire time interval. The resulting velocities were estimated via the gradient of position and time interval, respectively. Angle to nearest neighbor was calculated from the angular displacement between the direction of a focal fish and its nearest neighbor, for each fish in the school across the time interval (Rieucau et al., 2018). We computed school polarization as the sum of fish directions (velocity normalized by magnitude) divided by the total number of fish in the school (Attanasi et al., 2014; Tunstrøm et al., 2013). Finally, we calculated correlation length, the distance with which information about an individuals' behavior can be socially transmitted, in the same manner as Cavagna et al. (2010) and Handegard et al. (2012).

\section{(c) Statistical Analysis:}

We treated each school as an independent sample, with measured values for each individual fish averaged over the school. To determine whether behavioral responses to contrast level were different, we conducted a one-way Kruskal-Wallis rank sum test, and post-hoc comparisons were done via pairwise Wilcoxon rank sum tests, BenjaminiHochberg corrected for multiple comparisons. When comparing behaviors over the time 
of response, we used analysis of covariance (ANCOVA) to compare schooling metric values within contrast levels at each step in the time interval. Finally, a Principal Component Analysis (PCA) was conducted at each contrast level to visualize correlations in schooling metrics. Each variable was scaled to unit variance, with no rotation. All analyses were done using CRAN R statistical software (2018-07-02, Feather Spray).

\section{(d) Ethical Note:}

This research was conducted in accordance with the guidelines set forth in the Florida International University IACUC Animal Care and Use Protocols \#19-054. No human subjects were used in this study.

\section{Results}

Changes in contrast level were found to influence velocity-based components of the escape responses of schooling fish. With increased contrast the average speed over the entire behavioral response for all schools changed from 6 to $8.5 \mathrm{~ms}^{-1}$ and polarization from 0.37 to 0.46 , and the changes were significant for speed (Kruskal-Wallis, $\chi^{2}=21.7$, $\mathrm{p}<.001$ ) and polarization (Kruskal-Wallis, $\chi^{2}=7.9, \mathrm{p}=.048$ ) (Figure 2), respectively. Fish were more polarized at high contrast (Wilcoxon, $\mathrm{p}=0.018$ ), and swam faster in each increasing contrast level- $\sim 15 \%$ faster in medium-low contrast (Wilcoxon, $p=0.002$ ), $\sim 25 \%$ faster in medium-high contrast (Wilcoxon, $\mathrm{p}<0.001$ ), and $\sim 30 \%$ faster in high contrast (Wilcoxon, $\mathrm{p}<0.001$ ) compared to low contrast conditions (Figure 2), supporting the hypothesis that speed regulation is visually mediated. Change in contrast did not explain variability in nearest neighbor distance (Kruskal-Wallis, $\mathrm{p}>0.05$ ), nearest 
neighbor angle (Kruskal-Wallis, $\mathrm{p}>0.05$ ), or correlation length (Kruskal-Wallis, $\mathrm{p}>$ $0.05)$.

Over the time span of the behavioral response, schools in the high contrast condition exhibited a sharp increase in nearest neighbor distance and remained more loosely shoaled than schools in the lower contrast conditions (ANCOVA, $\mathrm{F}_{3,14557}=7.04$, $\mathrm{p}<0.001$ ) (Figure 3). Individual fish were also more closely aligned to their nearest neighbor in the high contrast condition; alignment angle to their nearest neighbor was greater at lower contrast levels (ANCOVA, $\left.\mathrm{F}_{3,14523}=8.166, \mathrm{p}<0.001\right)$. Average speed for all contrast levels began high, with an exponential decrease over the course of the response; at low contrasts, the average speed was lower than at higher contrasts (ANCOVA, $F_{3,14530}=61.421, p<0.001$ ), but decayed at the same rate (Figure 3). School polarization increased at all contrast levels over the course of the escape responses, however in low contrast conditions schools were less polarized than at higher contrast levels (ANCOVA, $\left.\mathrm{F}_{3,1570}=10.614, \mathrm{p}<0.001\right)$. Correlation length, the distance in which the behavior of one fish affects another, at all contrast levels, decreased over the course of the response interval yet no differences were found between the varying contrast levels $\left(\right.$ ANCOVA, $\left.F_{3,1496}=0.734, p=0.53\right)($ Figure 3).

Contrast level also influenced the correlation between schooling state metrics. At high contrast, polarization, angle, and nearest neighbor distance are loaded strongly on the first principal component, while speed loads strongly on the second and correlation length on the third principal component (Figure 4) (Appendix T1). Schooling state metrics in high contrast conditions were not highly correlated, and no groupings of correlated metrics occurred. In the medium-high contrast condition, polarization and 
angle maintain their strong loadings on the first principal component, with speed loading similarly between component $1 \& 2$. Correlation length and nearest neighbor distance load similarly on the primary and secondary principal component and are highly correlated. In the medium-low contrast condition, nearest neighbor distance is no longer highly correlated with correlation length but is now correlated with nearest neighbor distance. At low contrast, correlation length, nearest neighbor distance, and speed load strongly on the first principal component, while polarization and nearest neighbor angle load strongly on the second. At this lowest contrast level, speed, correlation length, and nearest neighbor distance are all highly correlated (Figure 4).

\section{Discussion}

Overall, our results demonstrate that fish schools exhibit weaker antipredator responses in low contrast environments that affect visual perception, suggesting sensory driven re-weighing of the individual interaction rules that drive collective state in dynamic environments. Consistent with studies exploring the effect of turbidity (Kimbell and Morrell, 2015), we found that school polarization decreased as well as speed in lower contrast environmental conditions; a result supporting the conclusion that abiotic environmental drivers have a direct effect on the schooling responses to predator attacks. The environments with decreased contrast appear to reproduce the effect of turbidity on predator detection, reducing a school's ability to adequately recognize the magnitude of predator threat (Ferrari et al., 2010) which results in a weaker behavioral response. The ability for schooling fish to adjust escape patterns relative to the perceived threat level, or “threat-sensitive response" (Brown et al., 2006; Ferrari et al., 2008; Rieucau et al., 2014), 
may be diminished when visual predator information is confounded, leading to decreased evasion success.

Additionally, our results provide supporting evidence to previous studies that demonstrate the role of vision and mechanosensation on schooling behavior, with vision particularly playing a pivotal role in determining the rules of interaction (StrandburgPeshkin et al., 2013) via control of velocity and orientation (Partridge and Pitcher, 1980). Our study also shows that vision affects the ability for a school to adequately respond to a sudden predator attack. During a predator attack, both the predator and other members of the school act as sources of visual sensory information, which provide threat information and collective information, respectively. In our experiment, the environmental conditions influence the sensory perception of the predator, which affect threat information, and not perception of other school members. This retention of collective information, in conjunction with the absence of manipulation of the lateral line system, suggests that the modified interaction rules resulting in decreases in polarization and speed are induced via the visual response to the perceived predator, rather than perception of the socially transmitted reaction wave. The lack of differences in other metrics of schooling tendency with decreasing visual perception indicates that the interaction rules are also regulated not just by vision, but by the lateral line system, which is used to maintain cohesion to neighbors once the escape response has been initiated (Partridge and Pitcher, 1980). The presence of other external sensory stimuli, in which food and alarm cues have been shown to affect collective dynamics in schools (Schaerf et al., 2017), may further influence the interaction mechanisms that control the magnitude of the antipredator 
response and potentially explain the persistence of prey success in vision compromised environments.

Variation in behavior over the course of the entire escape response further illustrate differences in schooling behavior at different contrast levels. In addition to the lower speeds and polarization with decreasing contrast, schools maintained tighter schooling at lower contrast levels across the entire behavioral response, which suggests that mechanosensory information becomes prioritized when visual perception is compromised. Similarly, fish were less locally aligned prior to attack at lower contrasts, which may reflect the need to maximize the collective visual field to enhance individual responses to neighbors and maximize escape success. The consistency in social information transfer ability across all levels of visual contrast highlights the potential for non-vision sensory modalities to contribute to the regulation of individual interaction rules to increase survival in dynamically changing conditions. As of now, we are unable to directly determine the exact contribution of the different sensory modalities to the interaction mechanisms that control the antipredator response, which provides an important avenue for further investigation.

Our study shows that the underlying metrics that constitute the schooling tendency shift in their relationships to each other due to the environment. The increased correlation between nearest neighbor distance, correlation length (information transfer), and speed as contrast level decreases further supports the idea that auxiliary sensory systems may be contributing more to the escape response than vision. As contrast drops, schooling state metrics that are to a lesser degree controlled by vision (nearest neighbor distance, correlation length), but rather by other sensory systems may become more 
correlated if the lateral line system is compensating for the loss of visual perception. Additionally, since the loss of visual perception directly influences speed, the grouping of speed with the non-visually mediated schooling metrics suggests that multiple sensory systems, not just vision, can control schooling behavior in conditions where information about the environment is unreliable. A predator in the same environment may experience a similar loss of visual perception, however visually-mediated piscivorous predators typically hunt in low-light conditions (Cerri, 1983), and can actively select when to attack; a prey school can only respond when an attack is initiated and correctly detected. In this study, we explored whether environmental conditions that influence visual perception of predators affects the antipredator schooling tendency of fish. We found that schooling behavior was modified in contrast-limited environments that influence the schools' ability to detect predators. In environments with lower visual contrast between a predator and its surroundings, escape responses of schooling fish were weaker, but only in behavioral metrics that are typically regulated by vision. When environmental conditions altered the ability to visually detect and respond to predators, the antipredator response may be driven more strongly by alternate sensory modalities (lateral line system) that act to increase probability of survival. Our results demonstrate that, in addition to the fact that environmental conditions modulate schooling behavior, antipredator responses of schools in visual information-constrained environments are not completely diminished but rather supplemented by other sensory systems. Understanding the contribution of the sensory systems of fish to schooling behavior will improve our knowledge of collective behavior. 
Animal Ethics \& Fieldwork Statement:

This research was conducted in accordance with the guidelines set forth in the Florida International University IACUC Animal Care and Use Protocol \#19-054

\section{Acknowledgements}

We thank Carlos Ruiz for technical and advisory help during the data collection phase.

\section{References}

Abrahams, M. V. and Kattenfeld, M. G. (1997). The role of turbidity as a constraint on predator-prey interactions in aquatic environments. Behavioral Ecology and Sociobiology 40, 169-174.

Attanasi, A., Cavagna, A., Del Castello, L., Giardina, I., Grigera, T., Jelić, A., Melillo, S., Parisi, L., Pohl, O., Shen, E., et al. (2014). Information transfer and behavioural inertia in starling flocks. Nature Physics 10, 691-696.

Bastien, R. and Romanczuk, P. (2020). A model of collective behavior based purely on vision. SCIENCE ADVANCES 10.

Berdahl, A., Torney, C. J., Ioannou, C. C., Faria, J. J. and Couzin, I. D. (2013). Emergent sensing of complex environments by mobile animal groups. Science 339, 574576 .

Bhattacharyya, K., McLean, D. L. and MacIver, M. A. (2017). Visual Threat Assessment and Reticulospinal Encoding of Calibrated Responses in Larval Zebrafish. Current Biology 27, 2751-2762.e6.

Brown, G. E., Rive, A. C., Ferrari, M. C. O. and Chivers, D. P. (2006). The dynamic nature of antipredator behavior: prey fish integrate threat-sensitive antipredator responses within background levels of predation risk. Behav Ecol Sociobiol 61, 916.

Cabrera, S. and Theobald, J. C. (2013). Flying fruit flies correct for visual sideslip depending on relative speed of forward optic flow. Frontiers in behavioral neuroscience 7, 76 . 
Cade, D., Carey, N., Domenici, P., Potvin, J. and Goldbogen, J. (2020). Predatorinformed looming stimulus experiments reveal how large filter feeding whales capture highly maneuverable forage fish. Proceedings of the National Academy of Sciences 117, 472-478.

Cavagna, A., Cimarelli, A., Giardina, I., Orlandi, A., Parisi, G., Procaccini, A., Santagati, R. and Stefanini, F. (2008). New statistical tools for analyzing the structure of animal groups. Mathematical Biosciences 214, 32-37.

Cavagna, A., Cimarelli, A., Giardina, I., Parisi, G., Santagati, R., Stefanini, F. and Viale, M. (2010). Scale-free correlations in starling flocks. Proceedings of the National Academy of Sciences 107, 11865-11870.

Cerri, R. D. (1983). The effect of light intensity on predator and prey behaviour in cyprinid fish: factors that influence prey risk. Animal Behaviour 31, 736-742.

Chamberlain, A. C. and Ioannou, C. C. (2019). Turbidity increases risk perception but constrains collective behaviour during foraging by fish shoals. Animal Behaviour $156,129-138$.

Collignon, B., Séguret, A. and Halloy, J. (2016). A stochastic vision-based model inspired by zebrafish collective behaviour in heterogeneous environments. Royal Society Open Science 3, 150473.

Conradt, L. (2012). Models in animal collective decision-making: information uncertainty and conflicting preferences. Interface Focus 2, 226-240.

Couzin, I. D. (2009). Collective cognition in animal groups. Trends in Cognitive Sciences $13,36-43$.

Couzin, I. D., Krause, J., James, R., Ruxton, G. D. and Franks, N. R. (2002). Collective memory and spatial sorting in animal groups. Journal of theoretical biology 218, $1-11$.

De Robertis, A., Ryer, C. H., Veloza, A. and Brodeur, R. D. (2003). Differential effects of turbidity on prey consumption of piscivorous and planktivorous fish. Canadian Journal of Fisheries and Aquatic Sciences 60, 1517-1526.

Delcourt, J. and Poncin, P. (2012). Shoals and schools: back to the heuristic definitions and quantitative references. Reviews in Fish Biology and Fisheries 22, 595-619.

Faucher, K., Parmentier, E., Becco, C., Vandewalle, N. and Vandewalle, P. (2010). Fish lateral system is required for accurate control of shoaling behaviour. Animal Behaviour 79, 679-687. 
Ferrari, M. C. O., Messier, F. and Chivers, D. P. (2008). Can prey exhibit threat-sensitive generalization of predator recognition? Extending the Predator Recognition Continuum Hypothesis. Proc. R. Soc. B 275, 1811-1816.

Ferrari, M. C. O., Lysak, K. R. and Chivers, D. P. (2010). Turbidity as an ecological constraint on learned predator recognition and generalization in a prey fish. Animal Behaviour 79, 515-519.

Figueiredo, B. R. S., Mormul, R. P., Chapman, B. B., Lolis, L. A., Fiori, L. F. and Benedito, E. (2016). Turbidity amplifies the non-lethal effects of predation and affects the foraging success of characid fish shoals. Freshwater Biology 61, 293300 .

Fotowat, H. and Gabbiani, F. (2011). Collision Detection as a Model for Sensory-Motor Integration. Annu. Rev. Neurosci. 34, 1-19.

Gautrais, J., Ginelli, F., Fournier, R., Blanco, S., Soria, M., Chaté, H. and Theraulaz, G. (2012). Deciphering Interactions in Moving Animal Groups. PLoS Computational Biology 8, e1002678.

Handegard, N. O., Boswell, K. M., Ioannou, C. C., Leblanc, S. P., Tjøstheim, D. B. and Couzin, I. D. (2012). The Dynamics of Coordinated Group Hunting and Collective Information Transfer among Schooling Prey. Current Biology 22, 1213-1217.

Hein, A. M., Gil, M. A., Twomey, C. R., Couzin, I. D. and Levin, S. A. (2018). Conserved behavioral circuits govern high-speed decision-making in wild fish shoals. Proc Natl Acad Sci USA 115, 12224-12228.

Herbert-Read, J. E., Buhl, J., Hu, F., Ward, A. J. and Sumpter, D. J. (2015). Initiation and spread of escape waves within animal groups. Royal Society open science 2, 140355.

Higham, T. E., Stewart, W. J. and Wainwright, P. C. (2015). Turbulence, Temperature, and Turbidity: The Ecomechanics of Predator-Prey Interactions in Fishes. Integrative and Comparative Biology 55, 6-20.

Hoare, D. J., Couzin, I. D., Godin, J.-G. J. and Krause, J. (2004). Context-dependent group size choice in fish. Animal Behaviour 67, 155-164.

Ioannou, C. C. (2017). Grouping and Predation. In Encyclopedia of Evolutionary Psychological Science (ed. Shackelford, T. K.) and Weekes-Shackelford, V. A.), pp. 1-6. Cham: Springer International Publishing.

Ioannou, C. C., Bartumeus, F., Krause, J. and Ruxton, G. D. (2011). Unified effects of aggregation reveal larger prey groups take longer to find. Proc. R. Soc. B 278, 2985-2990. 
Katz, Y., Tunstrøm, K., Ioannou, C. C., Huepe, C. and Couzin, I. D. (2011). Inferring the structure and dynamics of interactions in schooling fish. Proceedings of the National Academy of Sciences 108, 18720-18725.

Kent, M. I. A., Lukeman, R., Lizier, J. T. and Ward, A. J. W. (2019). Speed-mediated properties of schooling. R. Soc. open sci. 6, 181482.

Kimbell, H. S. and Morrell, L. J. (2015). Turbidity influences individual and group level responses to predation in guppies, Poecilia reticulata. Animal Behaviour 103, $179-185$.

Land, M. F. (1988). The optics of animal eyes. Contemporary Physics 29, 435-455.

Land, M. F. (2005). The optical structures of animal eyes. Current Biology 15, R319R323.

Land, M. F. and Nilsson, D.-E. (2012). Animal eyes. Oxford University Press.

Magurran, A. E. and Pitcher, T. J. (1987). Provenance, shoal size and the sociobiology of predator-evasion behaviour in minnow shoals. Proc. R. Soc. Lond. B 229, 439465.

Mogdans, J. and Bleckmann, H. (2012). Coping with flow: behavior, neurophysiology and modeling of the fish lateral line system. Biological Cybernetics 106, 627-642.

Montgomery, J., Coombs, S. and Halstead, M. (1995). Biology of the mechanosensory lateral line in fishes. Reviews in Fish Biology and Fisheries 5, 399-416.

Parrish, J. K., Viscido, S. V. and Grunbaum, D. (2002). Self-organized fish schools: an examination of emergent properties. The biological bulletin 202, 296-305.

Partridge, B. L. and Pitcher, T. J. (1980). The sensory basis of fish schools: Relative roles of lateral line and vision. Journal of Comparative Physiology? A 135, 315-325.

Peek, M. Y. and Card, G. M. (2016). Comparative approaches to escape. Current Opinion in Neurobiology 41, 167-173.

Pitcher, T. J. and Parrish, J. K. (1993). Functions of schooling behavior in fishes. The behavior of teleost fishes. Chapman and Hall, New York 363-440.

Rieucau, G., Boswell, K. M., De Robertis, A., Macaulay, G. J. and Handegard, N. O. (2014). Experimental Evidence of Threat-Sensitive Collective Avoidance Responses in a Large Wild-Caught Herring School. PLoS ONE 9, e86726.

Rieucau, G., Kiszka, J. J., Castillo, J. C., Mourier, J., Boswell, K. M. and Heithaus, M. R. (2018). Using unmanned aerial vehicle (UAV) surveys and image analysis in the 
study of large surface-associated marine species: a case study on reef sharks Carcharhinus melanopterus shoaling behaviour. J Fish Biol 93, 119-127.

Rosenthal, S. B., Twomey, C. R., Hartnett, A. T., Wu, H. S. and Couzin, I. D. (2015). Revealing the hidden networks of interaction in mobile animal groups allows prediction of complex behavioral contagion. Proc Natl Acad Sci USA 112, 46904695 .

Ryer, C. and Olla, B. 1. (1998). Effect of light on juvenile walleye pollock shoaling and their interaction with predators. Mar. Ecol. Prog. Ser. 167, 215-226.

Schaerf, T. M., Dillingham, P. W. and Ward, A. J. W. (2017). The effects of external cues on individual and collective behavior of shoaling fish. Sci. Adv. 3, e1603201.

Stewart, W. J., Nair, A., Jiang, H. and McHenry, M. J. (2014). Prey fish escape by sensing the bow wave of a predator. Journal of Experimental Biology 217, 43284336 .

Strandburg-Peshkin, A., Twomey, C. R., Bode, N. W. F., Kao, A. B., Katz, Y., Ioannou, C. C., Rosenthal, S. B., Torney, C. J., Wu, H. S., Levin, S. A., et al. (2013). Visual sensory networks and effective information transfer in animal groups. Current Biology 23, R709-R711.

Temizer, I., Donovan, J. C., Baier, H. and Semmelhack, J. L. (2015). A Visual Pathway for Looming-Evoked Escape in Larval Zebrafish. Current Biology 25, 1823 1834.

Tunstrøm, K., Katz, Y., Ioannou, C. C., Huepe, C., Lutz, M. J. and Couzin, I. D. (2013). Collective States, Multistability and Transitional Behavior in Schooling Fish. PLoS Computational Biology 9, e1002915.

Turner, G. F. and Pitcher, T. J. (1986). Attack Abatement: A Model for Group Protection by Combined Avoidance and Dilution. The American Naturalist 128, 228-240.

Utne-Palm, A. C. (2002). Visual feeding of fish in a turbid environment: Physical and behavioural aspects. Marine and Freshwater Behaviour and Physiology 35, 111128.

Viscido, S. V., Parrish, J. K. and Grünbaum, D. (2004). Individual behavior and emergent properties of fish schools: a comparison of observation and theory. Marine Ecology Progress Series 273, 239-250.

Ward, A. J. W., Herbert-Read, J. E., Sumpter, D. J. T. and Krause, J. (2011). Fast and accurate decisions through collective vigilance in fish shoals. Proceedings of the National Academy of Sciences 108, E27-E27. 
Zienkiewicz, A. K., Ladu, F., Barton, D. A. W., Porfiri, M. and Bernardo, M. D. (2018). Data-driven modelling of social forces and collective behaviour in zebrafish. Journal of Theoretical Biology 443, 39-51. 
Figures
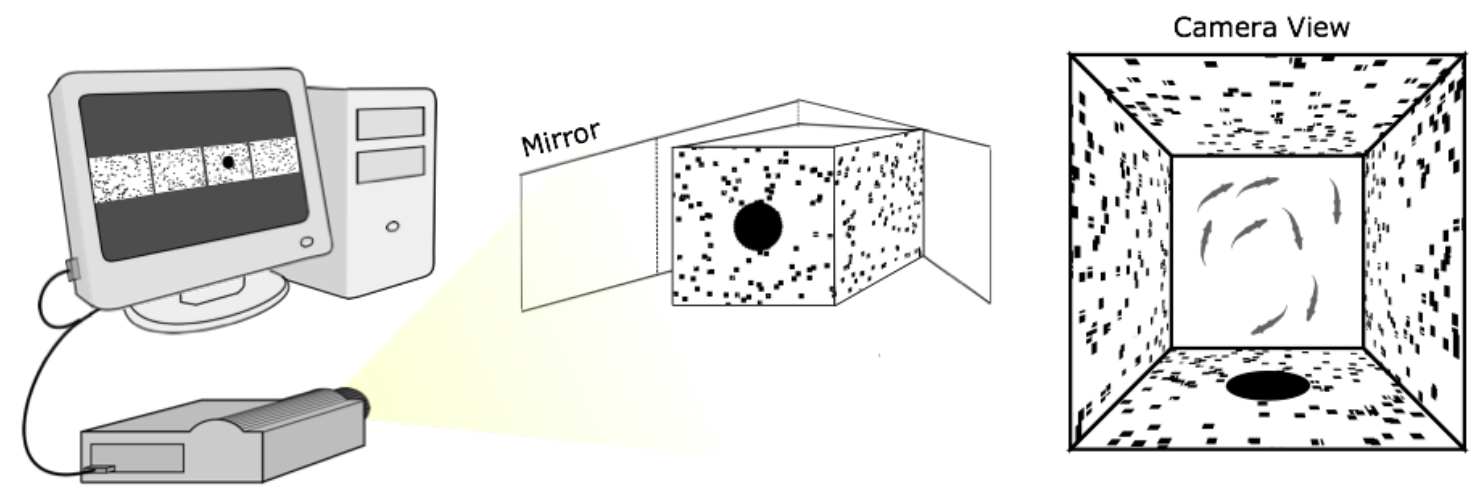

Figure (1): Experimental apparatus visualizing fish school in three dimensional virtual arena. Each school was subjected to four consecutive visual looming stimuli, with randomized direction, at a given contrast level. Once schools completed the experiment, they were returned to the tank. Subsequent trial sample randomly to ensure no two schools are exactly the same. 

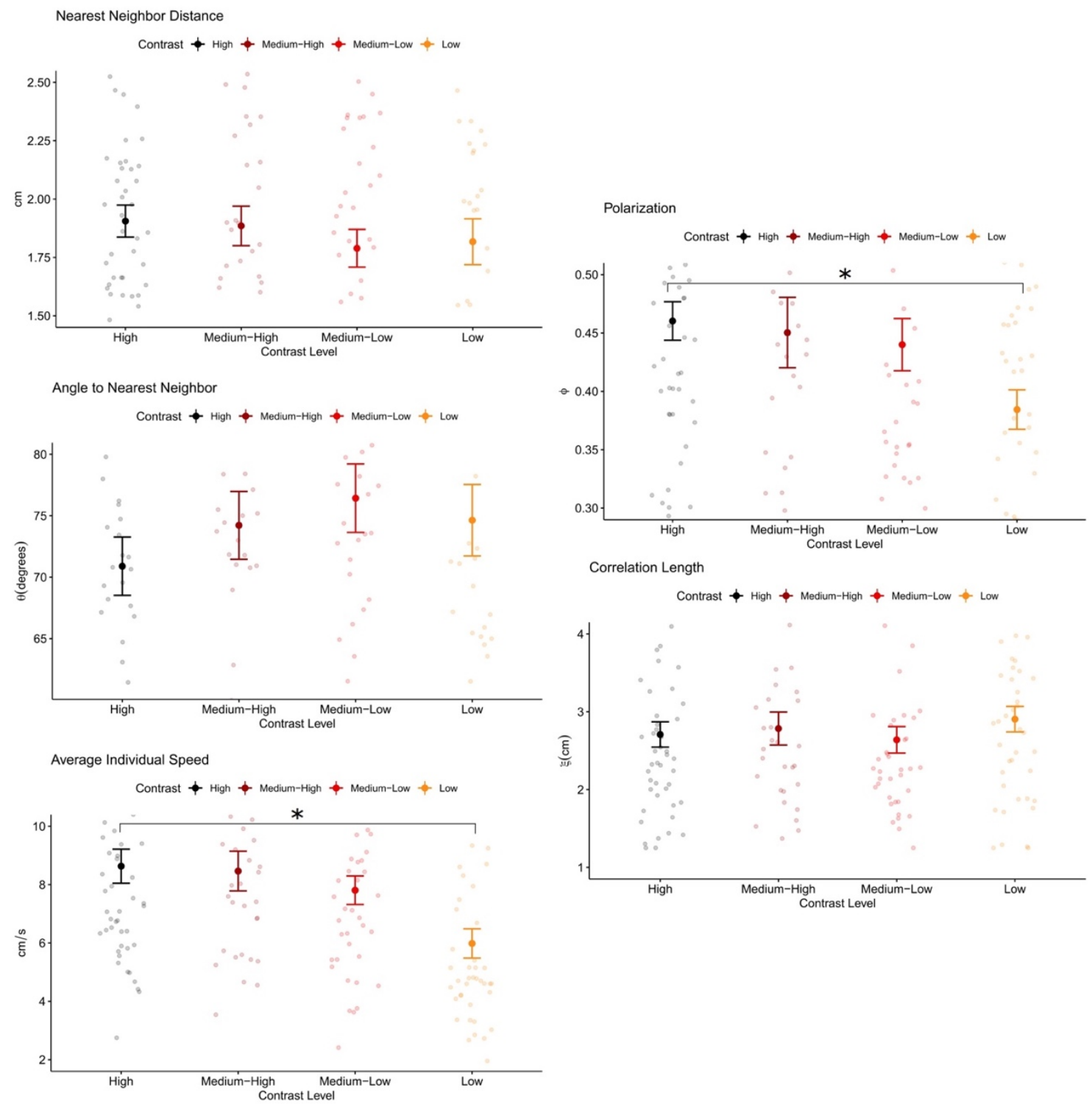

Figure (2): Mean values of five behavioral parameters in schooling minnow across four varying contrast levels, denoted by increasingly lighter color. Error bars represent standard error, and jitter points represent the raw values. Nearest neighbor distance, nearest neighbor alignment angle, and swimming speed were calculated from each fish in the school across the entire behavioral response time and averaged to provide one measurement per school tested. Polarization and Correlation strength were calculated per school, averaging across the entire time span of each behavioral response, providing one measurement per school tested. 
Nearest Neighbor Distance

Contrast - High - Medium-High - Medium-Low - Low

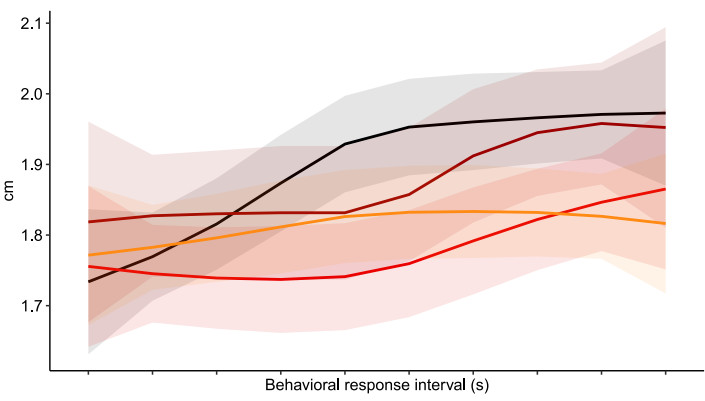

Alignment to Nearest Neighbor

Contrast - High - Medium-High - Medium-Low - Low

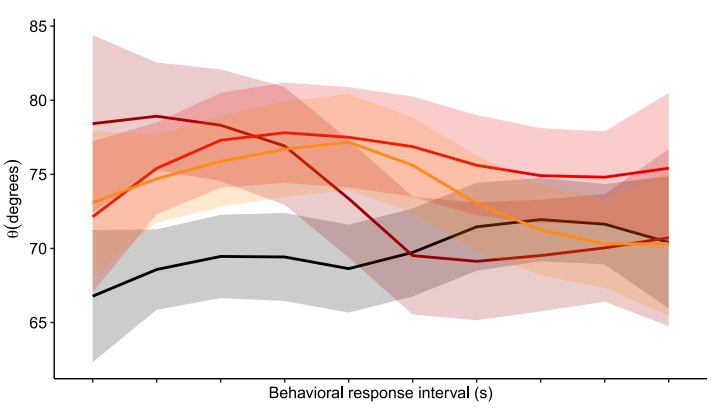

Average Individual Speed

Contrast - High - Medium-High - Medium-Low - Low

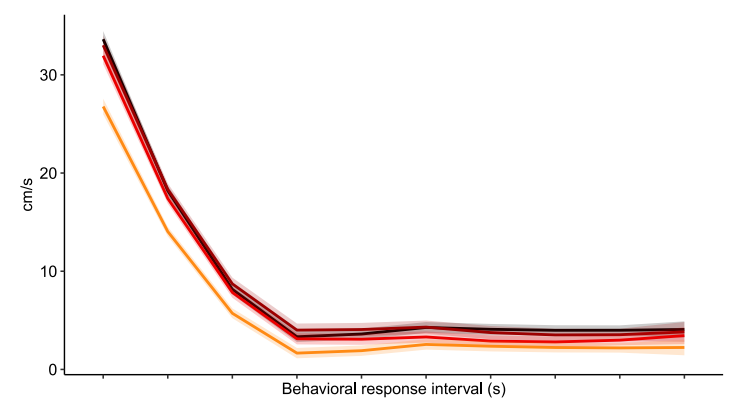

Polarization

contrast_level — High — Medium-High — Medium-Low — Low

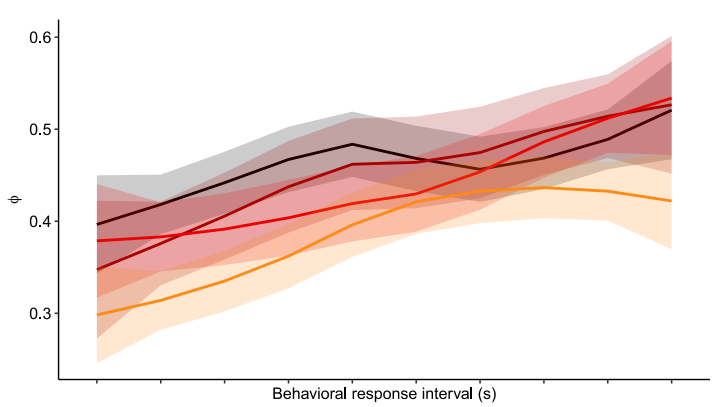

Correlation Length

contrast_level - High - Medium-High - Medium-Low - Low

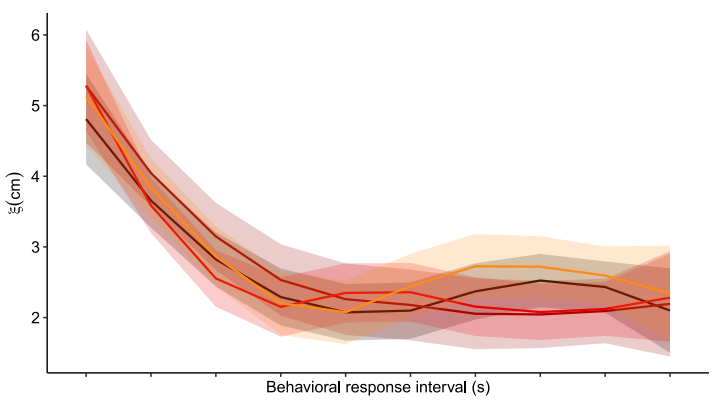

Figure (3): Evolution of five behavioral parameters in schooling minnow (species) along the time span of response to a looming stimulus across four contrast levels. The time span of the behavioral response varied per trial and were standardized by partitioning each behavioral response time span into 10 equidistant time intervals. Solid lines represent mean values of parameters, with contrast denoted by increasingly lighter color; standard error is represented by a similarly hued region bounding the mean value line. 

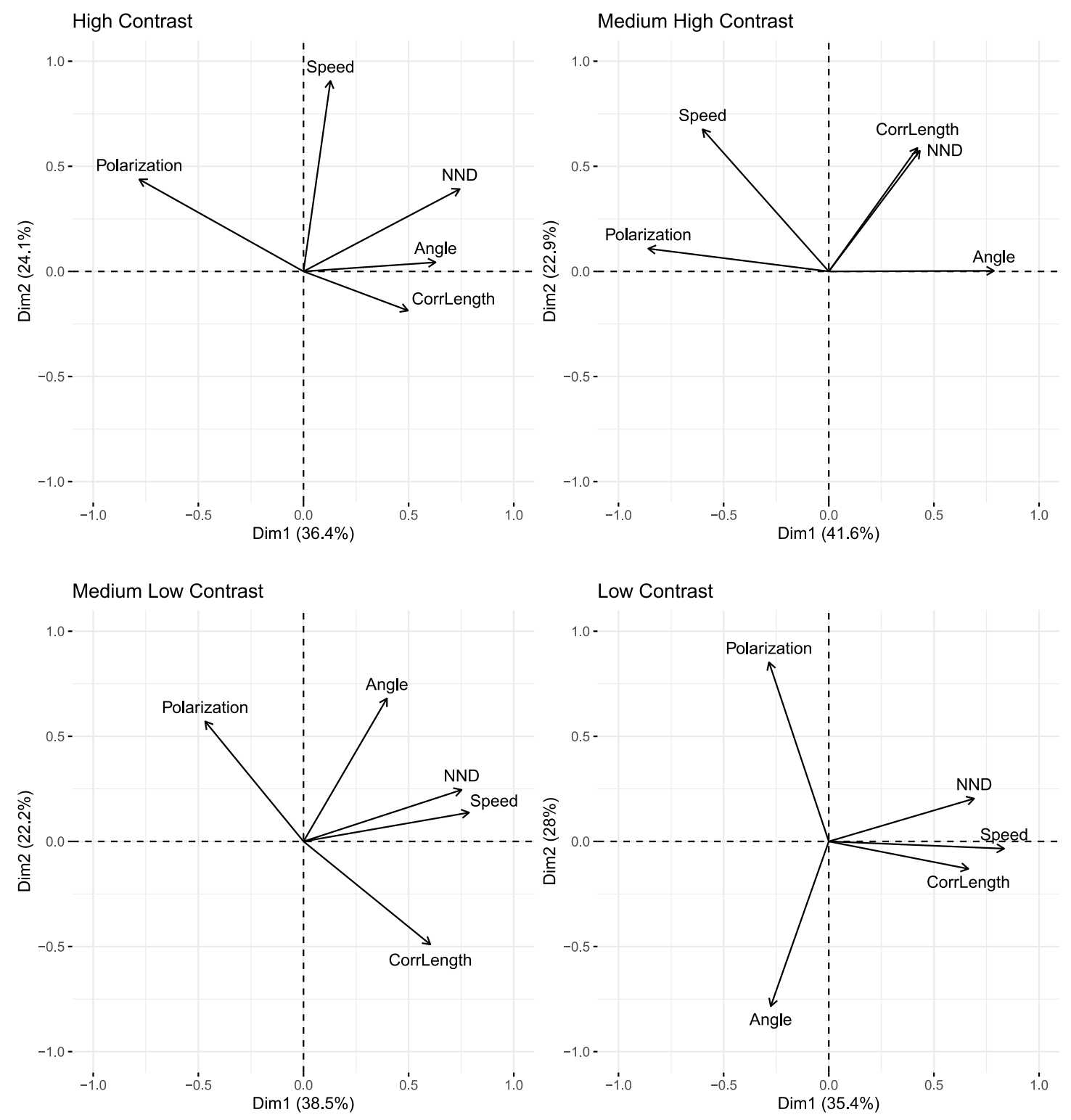

Figure (4): Principal Components Analysis of the five behavioral parameters of schooling minnow for each contrast level. Variable loadings on the first two principal components are shown for each contrast level tested. Each PCA was scaled to unit variance. 


\begin{tabular}{|c|c|c|c|c|c|}
\hline & $\mathrm{PC} 1$ & $\mathrm{PC} 2$ & PC3 & PC4 & PC5 \\
\hline Nearest Neighbor Distance & 0.7431607 & 0.39184375 & -0.06255103 & -0.480746 & 0.2431899 \\
\hline Nearest Neighbor Alignment & 0.6281712 & 0.04331195 & -0.61491323 & 0.4566845 & 0.1297919 \\
\hline Average Speed & 0.1280408 & 0.90657781 & 0.22740564 & 0.1868462 & -0.274039 \\
\hline Polarization & -0.7809628 & 0.43790675 & -0.02569501 & 0.1144974 & 0.4296102 \\
\hline Correlation Length & 0.496784 & -0.18619814 & 0.77211068 & 0.2735389 & 0.2180768 \\
\hline \multicolumn{6}{|l|}{ Medium-High Contrast } \\
\hline & $\mathrm{PC} 1$ & $\mathrm{PC} 2$ & $\mathrm{PC} 3$ & PC4 & PC5 \\
\hline Nearest Neighbor Distance & 0.4330552 & 0.57396491 & 0.62600662 & -0.29266947 & 0.07407915 \\
\hline Nearest Neighbor Alignment & 0.7856829 & 0.002839977 & 0.18215015 & 0.58026011 & 0.11319821 \\
\hline Average Speed & -0.5999326 & 0.675872129 & 0.01361152 & 0.29352611 & -0.31134376 \\
\hline Polarization & -0.8586388 & 0.108471405 & 0.15080096 & 0.138883 & 0.45710388 \\
\hline Correlation Length & 0.4217174 & 0.587658 & -0.65579023 & -0.08017737 & 0.20080652 \\
\hline \multicolumn{6}{|l|}{ Medium-Low Contrast } \\
\hline & $\mathrm{PC} 1$ & $\mathrm{PC} 2$ & PC3 & PC4 & PC5 \\
\hline Nearest Neighbor Distance & 0.7519345 & 0.2455167 & 0.1188536 & -0.43764956 & 0.410673461 \\
\hline Nearest Neighbor Alignment & 0.3968071 & 0.6805326 & -0.488485 & 0.37522094 & 0.003350618 \\
\hline Average Speed & 0.7873217 & 0.1373564 & 0.3464902 & -0.05865161 & -0.4876088 \\
\hline Polarization & -0.4676196 & 0.5711667 & 0.6216771 & 0.23047347 & 0.124499559 \\
\hline Correlation Length & 0.6032925 & -0.4901562 & 0.2028424 & 0.55386907 & 0.218789891 \\
\hline \multicolumn{6}{|l|}{ Low Contrast } \\
\hline & PC1 & $\mathrm{PC} 2$ & PC3 & PC4 & PC5 \\
\hline Nearest Neighbor Distance & 0.6911773 & 0.20390958 & 0.5670829 & 0.3112483 & -0.249472 \\
\hline Nearest Neighbor Alignment & -0.2760528 & -0.78401837 & 0.3880778 & 0.2888037 & 0.2740403 \\
\hline Average Speed & 0.8340566 & -0.03428064 & 0.1824436 & -0.4085915 & 0.3208453 \\
\hline Polarization & -0.2842564 & 0.85244538 & 0.136973 & 0.2375681 & 0.3425419 \\
\hline Correlation Length & 0.6637597 & -0.13026273 & -0.5997015 & 0.4111669 & 0.1172797 \\
\hline
\end{tabular}

Table (1): PCA variable loadings for each contrast condition. Schooling state parameter measurements are denoted in the first column, with principal components denoted along the first row. Scree plot analysis show that the first three principal components explain > $95 \%$ of the variability in the data. 
CHAPTER V

CONCLUSIONS \& FUTURE DIRECTIONS 
In my dissertation, I investigated the influence of dynamic environmental factors on the collective behavior of fish schools. In chapter II, I examined whether habitat context and complexity influenced the collective state of fish schools both prior to, and during predator attack. Results from this chapter show that habitat context, not predation, drives change in the collective state of fish schools. The habitats studied (both a marsh edge habitat and a higher complexity habitat) induced changes in the collective behavior of fish schools compared to an open water context, suggesting that the local environment plays a larger role than predation risk in structuring collective behavior. Chapter III builds off of these initial findings by observing fish schools at a water control structure, an anthropogenic structure that has both habitat complexity and increased risk of predation, to test whether the two effects influenced schooling behavior before and during predator attack. Results from this chapter demonstrate that collective behavior was influenced by the coupling of the two conditions, in a different manner than with habitat complexity alone. Cumulatively, chapters I and II emphasize the importance biotic and abiotic environmental factors play in structuring collective behavior, using in situ data to extract a true representation of these organisms in their natural, dynamic habitat. The goal of chapter IV was to return to the laboratory setting, to investigate collective behavior on a finer scale by isolating the visual system. By simulating turbidity changes by manipulating visual contrast, I could investigate whether environmental effects on a main sensory system affected collective responses to predators. Results from this chapter showed that schools maintained tighter schooling at lower contrast levels, suggesting that mechanosensory information becomes prioritized when visual perception is compromised. Furthermore, results indicate that multiple sensory systems can control 
schooling behavior in conditions where information about the environment is unreliable.

As a collective itself, these chapters advance our understanding of the role the environment plays on influencing collective animal behavior, as well as predator-prey dynamics in changing environments, and offers insights into this understanding from both a mechanistic and process based perspective.

Our understanding of collective animal behavior has grown a great deal in the last four decades. From the advancements in modeling of interaction mechanisms using sensory analogues to the use of advanced technologies to study collectives in situ, our knowledge of collective behavior and their emergent patterns has advanced from both the mechanistic and process based perspective. However, the results from this dissertation have shown that there are still many questions left to ask in order to decipher how collective patterns emerge and how the local environment may impact them. There exist a multitude of environmental factors that can affect collective behavior, from abiotic factors such as hypoxia, $\mathrm{pH}$, and local hydrology, to biotic factors such as predator density, local species richness, and coordinated hunting. Determining whether each of these factors affect collective behavior, and quantifying the extent to which they modulate behavior is a clear avenue for further investigation.

From a mechanistic perspective, it is imperative to fully elucidate how collective structure at the group level is derived from the rules of interaction; further studies in the effects of external factors on collective state will provide a more complete understanding of how collective patterns emerge from the mechanisms of individual interaction. In addition, determination of the control link between sensory input, rules of interaction, and collective responses is another line of study that is necessary to accomplish in the field. 
Deciphering whether one set of interaction rules, that simply tune the magnitude of the collective response to the magnitude of the sensory input, or multiple ones exist, with a control law acting to shift the set of interaction mechanisms depending on the magnitude of the sensory input. To date, no data exist suggesting that either case is more likely, but we can only address this question with knowledge of how collective state is modulated by external perturbations.

From a physiological perspective, the most interesting area for investigation is determining the relative contribution of each sensory system in the collective response. Establishing a baseline understanding of the weight of vision, mechanosensation, and olfaction to collective behavior would provide a fundamental mapping of the sensory systems to the interaction mechanisms that govern collective behavior. Furthermore, with an understanding of the contribution of each sensory system, we can begin to investigate how environmental effects influence collective responses by inducing shifts in the weighing of these sensory systems. This would provide a holistic understanding, from sensor to state to responses to perturbation, of the collective behavior of fish schools and their emergent properties.

In conclusion, my dissertation has contributed to the body of knowledge of collective animal behavior, helped our understanding of predator-prey dynamics in changing environments, and informed potential ways schooling fish will respond in the future to our rapidly changing climate and anthropogenic encroachment into the sea. 
VITA

\title{
IVAN IGNACIO RODRIGUEZ-PINTO
}

\author{
Born, Los Angeles, California
}

2011

B.Sc., Computational \& Systems Biology

University of California at Los Angeles

Los Angeles, California

2017

M.Sc., Biology

Florida International University

Miami, Florida

2017

Doctoral Candidate

Florida International University

Miami, Florida

2019

M.Sc., Electrical \& Computer Engineering

Florida International University

Miami, Florida

\section{PUBLICATIONS}

Caballero, J., Mazo, C., Rodriguez-Pinto, I., and Theobald, J.C., (2015). A visual horizon affects steering responses during flight in fruit flies. Journal of Experimental Biology, 218, no. 18: 2942-2950.

D'Elia, M., Warren, J.D., Rodriguez-Pinto, I., Sutton, T.T., Cook, A., and Boswell, K.M., (2016). Diel variation in the vertical distribution of deep-water scattering layers in the Gulf of Mexico. Deep Sea Research Part I: Oceanographic Research Papers 115: 91-102.

Svendsen, M.B.S., Domenici, P., Marras, S., Krause, J., Boswell,K.M., Rodriguez-Pinto, I., Wilson A.D.M., et al. (2016) Maximum swimming speeds of sailfish and three other large marine predatory fish species based on muscle contraction time and stride length: a myth revisited. Biology Open 5, no. 10: 1415-1419.

Rodriguez-Pinto, I., Rieucau, G., Handegard, NO., and Boswell, KM., (2020), Environmental Context Elicits Behavioural Modification of Collective State in Schooling Fish. Animal Behaviour, https://doi.org/10.1016/j.anbehav.2020.05.002

Kilfoil, J.P., Rodriguez-Pinto, I.I., Kiszka,J.J., Heithaus,M.M., Zhang,Y., Roa, C.C., Ailloud, L.E., Campbell, M.D., and Wirsing, A.J., (2020). Using unmanned aerial vehicles and machine learning to improve sea cucumber density estimation in shallow habitats. In Press at ICES Journal of Marine Science. 